\title{
Interval type-2 fuzzy multi-attribute decision-making approaches for evaluating the service quality of Chinese commercial banks
}

\author{
Guolin Tang ${ }^{a}$, Francisco Chiclana ${ }^{b, c}$, XiangChun Lin ${ }^{d}$, Peide Liu ${ }^{a^{*}}$ \\ ${ }^{a}$ School of Management Science and Engineering, Shandong University of Finance and Economics, Shandong, China \\ ${ }^{b}$ Institute of Artificial Intelligence, De Montfort University, Leicester, UK \\ ${ }^{c}$ Andalusian Research Institute on Data Science and Computational Intelligence, University of Granada, Granada, Spain \\ ${ }^{d}$ Shandong Provincial Institute of Education Sciences, Shandong, China \\ *Corresponding author: Tel: +86-531-82222188,E-mail: Peide.liu@gmail.com
}

\begin{abstract}
In today's world, with increased competition, the service quality of Chinese commercial banks is recognized as a major factor that is responsible for enhancing competitiveness. Therefore, it is necessary to evaluate and analyse the service quality of Chinese commercial banks to realize their stable development. The service quality evaluation could be recognized as a multi-attribute decision-making (MADM) problem with multiple assessment attributes, both being of a qualitative and quantitative nature. Owing to the growing complexity and high uncertainty of the financial environment, the assessments of attributes cannot always possibly express using a real and/or type-1 fuzzy number. Additionally, a heterogeneous relationship often exists among the attributes under many real decision cases. In this study, we create two MADM approaches to handle decision-making problems with interval type-2 fuzzy numbers (IT2FNs) and offer their application to service quality evaluations of commercial banks problems. Specifically, we first define some operations on IT2FNs based on Archimedean T-norms (ATs) and develop a bi-directional projection measure of IT2FNs. Next, by combining the generalized Banzhaf index, the Choquet integral and IT2FNs, we propose the interval type-2 fuzzy Archimedean Choquet (IT2FAC) operator, the Banzhaf IT2FAC (BIT2FAC) operator and the 2-additive BIT2FAC (2ABIT2FAC) operator. Then, we establish two optimal models for deriving the weights of attributes based on a bi-directional projection measure of IT2FNs and Banzhaf function. Finally, we create two novel MADM methods under interval type-2 fuzzy contexts, where an illustrative case concerning the service quality evaluation of Chinese commercial banks is used to explain the created MADM approaches.
\end{abstract}

Keywords: multiple attributes analysis; service quality evaluation; Choquet integral; interval type-2 fuzzy numbers; Archimedean T-norms

\section{Introduction}

A commercial bank is a financial institution that provides financial service products. Similar to general industrial and commercial enterprises, such a bank pursues the maximization of enterprise profit on the premise of satisfying customer demands. Commercial banks have played a crucial role in China's development and competitiveness and have received widespread attention in recent years [1] [7] [50], with the sector having experienced a significant increase. According to the statistics of the China Banking Regulatory Commission, as of the end of 2018, the total assets of the commercial bank sector increased by 55.5\% compared to that in 2014, reaching RMB 203.4 trillion. During the same period, the total liabilities of the commercial bank sector also increased by $54.4 \%$ compared to that in 2014 , reaching RMB 187.1 trillion. Although Chinese commercial banks develop rapidly, they are facing fierce competition and challenges from advanced foreign banks and internet finance due to the 
incredible growth of globalization and the quick advancement of internet technology. Consequently, it is becoming more important to enhance the competitiveness of Chinese commercial banks to maintain stable development. Karatepe et al. [20] pointed out that the delivery of high service quality to customers provides enterprises with an opportunity to differentiate themselves in competitive markets. Moreover, Duncan and Elliot [8] argued that providing high quality service to customers can lead to customer satisfaction and customer loyalty, which are key to a bank's long-term success and competitiveness. Based on these arguments, it can be seen that commercial banks should put more emphasis on service quality to enhance their competitiveness. Therefore, it is necessary to establish an effective service quality evaluation model of Chinese commercial banks with the goal of enhancing their competitiveness.

The service quality evaluation of commercial banks could be recognized as a multi-attribute decision-making (MADM) problem with multiple assessment attributes [36] [46] [48], including technology level, service attitude, consultation service, waiting area environment, window opening situation, operational capacity, security and financial product. In real-world service quality evaluation problems, most of the assessment detailed information is unknown, and many factors are influenced by uncertainty. Consequently, classical type-1 fuzzy numbers (T1FNs) may be inappropriate to handle actual cases in which it is unreasonable to utilize an accurate membership degree (MD) for an uncertain item [18]. In this situation, type-2 fuzzy numbers (T2FNs) [47], which consist of primary MD and secondary MD, can be regarded as a feasible tool to cope with higher uncertainty [28]. Interval T2FNs (IT2FNs) are the most extensively utilized; MDs of IT2FNs take the form of crisp intervals, which make the calculations related to IT2FNs manageable. Therefore, the theory of IT2FNs is easy to utilize in practical management application domains [40] [41] [43].

There are usually two different ways to cope with interval type-2 fuzzy (IT2F) MADM problems: developing extended traditional decision-making approaches [17] [35] and/or developing aggregation operators' methods [24] [25]. Aggregation operators' methods are superior to extended traditional decision-making approaches because the aggregation operators' methods provide comprehensive values of alternatives along with a ranking of alternatives, whereas extended traditional decision-making approaches only rank alternatives. Thus, aggregation operators' methods are now receiving increased attention in the domain of decision-making [10] [21] [23]. For the aggregation operators' methods, generally speaking, the following two aspects need to be considered:

1) The operational laws. At first, most aggregation operators of IT2FNs utilized the arithmetic operational laws of IT2FNs to carry out the combination process [4]. Later, Gong et al. [13] studied some novel operational laws of IT2FNs based on Einstein operations. However, both operation approaches have two weaknesses. First, for the multiplication operation, the effect of a larger membership function on the decision result is omitted. Second, for the power multiplication operation, the influence of real value on the membership function of the element is neglected. To overcome these weaknesses, some improved operational laws of IT2FNs were explored based on special cases of Archimedean t-norm and t-conorm (ATT). For instance, Hu et al. [18] explored some algebraic operations of trapezoidal IT2FNs, Qin [32] designed some novel operations of symmetric triangular IT2FNs based on algebraic 
operations, while Qin and Liu [33] investigated some Frank operational laws of IT2FNs. Clearly, ATT can generate general and versatile operations, such as algebraic operations, Einstein operations, and Frank operations. Therefore, it is key to offer the operations of IT2FNs based on the ATT.

2) Functional operators. Regarding the extended power averaging operator [26], extended Bonferroni mean [14], extended Heronian mean [43], extended Hamy mean [32] and extended Maclaurin symmetric mean [42], although some of the aforementioned operators capture the interrelationship of the attributes, they assume that each attribute is associated with the remaining attributes, i.e., they can only consider the homogeneous relationship of the attributes [9]. However, in real cases, the attributes do not always have a homogeneous relationship. There may be cases where a heterogeneous relationship exists among the attributes [38]. For instance, when evaluating the service quality of a commercial bank, we may consider the following attributes: service attitude, consultation service, operational capacity and security. Here, service attitude and consultation service can be regarded as negatively related attributes, consultation service and security can be regarded as independent, while service attitude and operational capacity can be regarded as positively related attributes. Consequently, the existing aggregation operators of IT2FNs may not be able to handle this type of MADM problem. Nevertheless, the Choquet integral [15] can capture the heterogeneous relationship of the attributes because it is based on the fuzzy measure (FM) [37], which is an effective tool to model the positive interaction, negative interaction or independence of the attributes [2]. It is worth mentioning that the Choquet integral only considers the heterogeneous relationship between adjacent combinations of attributes. To globally capture the relationship of the attributes, Meng et al. [31] combined the generalized Banzhaf index and the Choquet integral and presented some novel Choquet operators of intuitionistic fuzzy sets. Recently, many researchers have extended the Choquet integral to different kinds of fuzzy environments [22] [24] [39]. To the best of our knowledge, no attempt has been made to extend the Choquet integral with the generalized Banzhaf index for handling IT2FSs; this extension is the focus of the present article.

As described above, the service quality evaluation procedure includes both uncertainty information aggregation and the interactive characteristics of the attributes. Consequently, the T1FNs may be inappropriate to handle real-world cases due to the growing uncertainty of the service quality evaluation problem, and the IT2FNs can be utilized instead. In addition, the ATT can provide general and versatile operations. Simultaneously, the Choquet integral with the generalized Banzhaf index can consider the heterogeneous relationship of the attributes. Therefore, it is justifiable to combine the ATT, the Choquet integral and the generalized Banzhaf index for handling service quality evaluation problems under the IT2FN environment. Additionally, a lack of knowledge and the experts' limited expertise about service quality evaluation problems mean that the information on attributes may not be always completely known. In this case, we need to obtain the attributes' weights first. Thus, we establish optimal models, based on the developed bi-directional projection measure of IT2FNs and the Banzhaf function for optimal FMs on the attributes set to derive the attributes' 
weights. Motivated by these ideas, we propose two IT2F MADM approaches based on the ATT, the Choquet integral, and the generalized Banzhaf index for handling real-life service quality evaluation problems with incomplete weights information. As a conclusion of the above statements, this article has four sub-objectives:

1) To define novel operations of IT2FNs to address the weaknesses of the existing operations of IT2FNs [4] [13] and to generate general and versatile operations.

2) To propose the interval type-2 fuzzy Archimedean Choquet (IT2FAC) operator, the Banzhaf IT2FAC (BIT2FAC) operator and the 2-additive BIT2FAC (2ABIT2FAC) operator to reflect the positively related, negatively related or independent characteristics of the attributes and to effectively fuse IT2F information.

3) To construct linear programming models for optimal FMs on the attributes set, based on the developed bi-directional projection measure of IT2FNs and the Banzhaf function, to objectively determine the attributes' weights.

4) To create novel MADM approaches based on the IT2F Choquet integral operators and the constructed optimal models to overcome the drawbacks of the existing methods [13] [14] [18] and to handle service quality evaluation problems.

To achieve the above research goals, the rest of this manuscript is organized as follows: Section 2 introduces some basic knowledge of IT2FNs and Choquet integral. Section 3 proposes the IT2FAC operator, the BIT2FAC operator and the 2ABIT2FAC operator for IT2FNs, and analyses their corresponding properties. Section 4 defines a novel bi-directional projection measure of IT2FNs and constructs optimal models to derive the attributes' weights. Section 5 creates two algorithms to cope with MADM problems under IT2FNs environment. In Section 6, a practical case is given to delineate the applicability and usefulness of the created approaches. Section 7 concludes this study.

\section{Preliminaries}

In this section, we shall introduce some basic knowledge related to IT2FNs, the ATT and the Choquet integral that will be utilized later in the article.

\subsection{IT2FNs}

Definition 1 [34]. An IT2FN is called trapezoidal IT2FN (See Fig. 1) when the upper membership function (MF) and the lower MF are both trapezoidal fuzzy values, i.e.,

$$
A=\left(A^{u}, A^{l}\right)=\left(\left(a_{1}^{u}, a_{2}^{u}, a_{3}^{u}, a_{4}^{u} ; h_{1}\left(A^{u}\right), h_{2}\left(A^{u}\right)\right),\left(a_{1}^{l}, a_{2}^{l}, a_{3}^{l}, a_{4}^{l} ; h_{1}\left(A^{l}\right), h_{2}\left(A^{l}\right)\right)\right),
$$

where $a_{1}^{l}, a_{2}^{l}, a_{3}^{l}, a_{4}^{l}, a_{1}^{u}, a_{2}^{u}, a_{3}^{u}$ and $a_{4}^{u}$ represent the reference points of the trapezoidal IT2FN $A$, subject $\quad$ to $0 \leq a_{1}^{l} \leq a_{2}^{l} \leq a_{3}^{l} \leq a_{4}^{l} \leq 1, \quad 0 \leq a_{1}^{u} \leq a_{2}^{u} \leq a_{3}^{u} \leq a_{4}^{u} \leq 1 ; \quad h_{j}\left(A^{l}\right) \quad$ and $\quad h_{j}\left(A^{u}\right) \quad(j=1,2)$ indicate the MFs of the elements $a_{j+1}^{l}$ and $a_{j+1}^{u}$, respectively, verifying $0 \leq h_{j}\left(A^{l}\right), h_{j}\left(A^{u}\right) \leq 1$. 


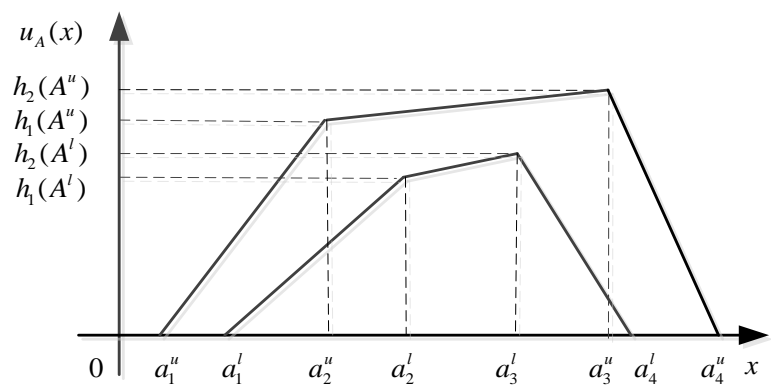

Fig. 1. A trapezoidal IT2FN $A$

To rank trapezoidal IT2FNs, the following ranking functions were defined by Qin and Liu [34].

Definition 2 [34]. It is assumed that $A=\left(A^{u}, A^{l}\right)$ is a trapezoidal IT2FN. Then, the arithmetic average ranking function $R_{1}(A)$, the geometric average ranking function $R_{2}(A)$ and the harmonic average ranking function $R_{3}(A)$ of $A=\left(A^{u}, A^{l}\right)$ are:

$$
\begin{gathered}
R_{1}(A)=\left(\frac{a_{1}^{u}+a_{4}^{u}}{2}+\frac{\sum_{j=1}^{2}\left(h_{j}\left(A^{u}\right)+h_{j}\left(A^{l}\right)\right)}{4}\right) \times \frac{\sum_{k=1}^{4}\left(a_{k}^{u}+a_{k}^{l}\right)}{8}, \\
R_{2}(A)=\left(\sqrt{a_{1}^{u} a_{4}^{u}}+\left(\prod_{j=1}^{2} h_{j}\left(A^{u}\right) h_{j}\left(A^{l}\right)\right) \times \times \sqrt[8]{\prod_{k=1}^{4} a_{k}^{u} a_{k}^{l}},\right. \\
R_{3}(A)=\left(\frac{2 a_{1}^{u} a_{4}^{u}}{a_{1}^{u}+a_{4}^{u}}+\frac{4}{\left.\sum_{j=1}^{2} \frac{h_{j}\left(A^{u}\right)+h_{j}\left(A^{l}\right)}{h_{j}\left(A^{u}\right) h_{j}\left(A^{l}\right)}\right) \times \frac{8}{\sum_{k=1}^{4}\left(\frac{1}{a_{k}^{u}}+\frac{1}{a_{k}^{l}}\right)} .} .\right.
\end{gathered}
$$

To improve the reliability of the decision-making process, Qin and Liu [34] further defined the following combined ranking function of trapezoidal IT2FN.

Definition 3 [34]. It is assumed that $A=\left(A^{u}, A^{l}\right)$ is a trapezoidal IT2FN. Then, the combined ranking function of $A=\left(A^{u}, A^{l}\right)$ is:

$$
R(A)=\varsigma_{1} R_{1}(A)+\varsigma_{2} R_{2}(A)+\varsigma_{3} R_{3}(A) .
$$

where $\xi_{j}=q^{j-1} / \sum_{k=0}^{2} q^{k}(j=1,2,3)$, multiplier $q$ is the solution of $2 \delta q^{2}+\sum_{j=2}^{3}(2 \delta-j+1) q^{3-j}=0$, and $\delta=\sum_{i=1}^{3} \frac{3-i}{2}\left(\frac{\ln 2+R_{i}(A) \ln R_{i}(A)}{\sum_{i=1}^{3} \ln 2+R_{i}(A) \ln R_{i}(A)}\right)$. For simplicity, it is assumed $\delta=0.5$ in this paper.

Qin and Liu [34] gave the following ranking technique to compare two trapezoidal IT2FNs.

Definition 4 [34]. It is assumed that $A_{1}=\left(A_{1}^{u}, A_{1}^{l}\right)$ and $A_{2}=\left(A_{2}^{u}, A_{2}^{l}\right)$ are two trapezoidal IT2FNs.

(1) If $R\left(A_{1}\right)>R\left(A_{2}\right)$, then $A_{1}>A_{2}$; 
(2) If $R\left(A_{1}\right)=R\left(A_{2}\right)$, then $A_{1}=A_{2}$.

(3) If $R\left(A_{1}\right)<R\left(A_{2}\right)$, then $A_{1}<A_{2}$.

\subsection{The operations of IT2FNs based on T-norm}

Intersection (meet) and union (join) of IT2FNs are defined based on ATT, respectively. Given a monotonic decreasing function $g(t):(0,1] \rightarrow R^{+}$such that $g^{-1}(t): R^{+} \rightarrow(0,1]$ verifies: $\lim _{t \rightarrow \infty} g^{-1}(t)=0$ and $g^{-1}(0)=1$, then, $T(x, y)=g^{-1}(g(x)+g(y))$ is a T-norm with $g(t)$ as its additive generator [6]. Table 1 shows four of the most used families of ATs and their additive generators.

Table 1. ATs and their additive generators

\begin{tabular}{ccc}
\hline Name & Formulas of the t-norms & Additive generators \\
\hline Algebraic $T_{A}$ & $T_{A}(x, y)=x y$ & $g(t)=-\ln t$ \\
Einstein $T_{E}$ & $T_{E}(x, y)=\frac{x y}{1+(1-x)(1-y)}$ & $g(t)=\ln \frac{2-t}{t}$ \\
Frank $T_{F, \gamma}(x, y)$ & $T_{F, \gamma}(x, y)=\log _{\gamma}\left(1+\frac{\left(\gamma^{x}-1\right)\left(\gamma^{y}-1\right)}{\gamma-1}\right)=\left\{\begin{array}{l}-\ln t, \gamma=1 \\
-\ln \frac{\gamma-1}{\gamma^{t}-1}, \gamma \neq 1\end{array}\right.$ \\
Aczel-Alsina $T_{A A, \gamma}(x, y)$ & $T_{A A, \gamma}(x, y)=e^{-\left((-\ln x)^{\gamma}+(-\ln y)^{\gamma}\right)^{1 / \gamma}}, \gamma>0$ & $g(t)=(-\ln t)^{\gamma}, \gamma>0$
\end{tabular}

Given a T-norm $T(x, y)$, the following operations of trapezoidal IT2FNs are defined.

Definition 5. It is assumed that $A_{1}=\left(A_{1}^{u}, A_{1}^{l}\right)$ and $A_{2}=\left(A_{2}^{u}, A_{2}^{l}\right)$ are any two trapezoidal IT2FNs.

Given a T-norm $T(x, y)$ with additive generator function:

$$
\begin{gathered}
A_{1} \otimes A_{2}=\left(\left(T\left(a_{11}^{u}, a_{21}^{u}\right), T\left(a_{12}^{u}, a_{22}^{u}\right), T\left(a_{13}^{u}, a_{23}^{u}\right), T\left(a_{14}^{u}, a_{24}^{u}\right) ; T\left(h_{1}\left(A_{1}^{u}\right), h_{1}\left(A_{2}^{u}\right)\right), T\left(h_{2}\left(A_{1}^{u}\right), h_{2}\left(A_{2}^{u}\right)\right)\right),\right. \\
\left.\left(T\left(a_{11}^{l}, a_{21}^{l}\right), T\left(a_{12}^{l}, a_{22}^{l}\right), T\left(a_{13}^{l}, a_{23}^{l}\right), T\left(a_{14}^{l}, a_{24}^{l}\right) ; T\left(h_{1}\left(A_{1}^{l}\right), h_{1}\left(A_{2}^{l}\right)\right), T\left(h_{2}\left(A_{1}^{l}\right), h_{2}\left(A_{2}^{l}\right)\right)\right)\right) \\
=\left(\left(g^{-1}\left(g\left(a_{11}^{u}\right)+g\left(a_{21}^{u}\right)\right), g^{-1}\left(g\left(a_{12}^{u}\right)+g\left(a_{22}^{u}\right)\right), g^{-1}\left(g\left(a_{13}^{u}\right)+g\left(a_{23}^{u}\right)\right), g^{-1}\left(g\left(a_{14}^{u}\right)+g\left(a_{24}^{u}\right)\right) ;\right.\right. \\
\left.g^{-1}\left(g\left(h_{1}\left(A_{1}^{u}\right)\right)+g\left(h_{1}\left(A_{2}^{u}\right)\right)\right), g^{-1}\left(g\left(h_{2}\left(A_{1}^{u}\right)\right)+g\left(h_{2}\left(A_{2}^{u}\right)\right)\right)\right),\left(g^{-1}\left(g\left(a_{11}^{l}\right)+g\left(a_{21}^{l}\right)\right), g^{-1}\left(g\left(a_{12}^{l}\right)+g\left(a_{22}^{l}\right)\right),\right. \\
\left.\left.g^{-1}\left(g\left(a_{13}^{l}\right)+g\left(a_{23}^{l}\right)\right), g^{-1}\left(g\left(a_{14}^{l}\right)+g\left(a_{24}^{l}\right)\right) ; g^{-1}\left(g\left(h_{1}\left(A_{1}^{l}\right)\right)+g\left(h_{1}\left(A_{2}^{l}\right)\right)\right), g^{-1}\left(g\left(h_{2}\left(A_{1}^{l}\right)\right)+g\left(h_{2}\left(A_{2}^{l}\right)\right)\right)\right)\right), \\
\left(A_{1}\right)^{\lambda}=\left(\left(g^{-1}\left(\lambda g\left(a_{11}^{u}\right)\right), g^{-1}\left(\lambda g\left(a_{12}^{u}\right)\right), g^{-1}\left(\lambda g\left(a_{13}^{u}\right)\right), g^{-1}\left(\lambda g\left(a_{14}^{u}\right)\right) ; g^{-1}\left(\lambda g\left(h_{1}\left(A_{1}^{u}\right)\right)\right), g^{-1}\left(\lambda g\left(h_{2}\left(A_{1}^{u}\right)\right)\right)\right),\right. \\
\left.\left(g^{-1}\left(\lambda g\left(a_{11}^{l}\right)\right), g^{-1}\left(\lambda g\left(a_{12}^{l}\right)\right), g^{-1}\left(\lambda g\left(a_{13}^{l}\right)\right), g^{-1}\left(\lambda g\left(a_{14}^{l}\right)\right) ; g^{-1}\left(\lambda g\left(h_{1}\left(A_{1}^{l}\right)\right)\right), g^{-1}\left(\lambda g\left(h_{2}\left(A_{1}^{l}\right)\right)\right)\right)\right) .
\end{gathered}
$$

Note that Eqs. (6)-(7) are the general formulations with additive generator function $g(t)$. In what follows, we provide some special cases using the additive generators of Table 1.

(1) For the algebraic T-norm $T_{A}(x, y)$, Eqs. (6)-(7) become the operations defined by Hu et al. [18] $(\lambda>0)$ :

$$
\begin{aligned}
A_{1} \otimes A_{2}= & \left(\left(a_{11}^{u} a_{21}^{u}, a_{12}^{u} a_{22}^{u}, a_{13}^{u} a_{23}^{u}, a_{14}^{u} a_{24}^{u} ; h_{1}\left(A_{1}^{u}\right) h_{1}\left(A_{2}^{u}\right), h_{2}\left(A_{1}^{u}\right) h_{2}\left(A_{2}^{u}\right)\right),\right. \\
& \left.\left(a_{11}^{l} a_{21}^{l}, a_{12}^{l} a_{22}^{l}, a_{13}^{l} a_{23}^{l}, a_{14}^{l} a_{24}^{l} ; h_{1}\left(A_{1}^{l}\right) h_{1}\left(A_{2}^{l}\right), h_{2}\left(A_{1}^{l}\right) h_{2}\left(A_{2}^{l}\right)\right)\right),
\end{aligned}
$$




$$
\begin{aligned}
\left(A_{1}\right)^{\lambda}= & \left(\left(\left(a_{11}^{u}\right)^{\lambda},\left(a_{12}^{u}\right)^{\lambda},\left(a_{13}^{u}\right)^{\lambda},\left(a_{14}^{u}\right)^{\lambda} ;\left(h_{1}\left(A_{1}^{u}\right)\right)^{\lambda},\left(h_{2}\left(A_{1}^{u}\right)\right)^{\lambda}\right),\right. \\
& \left.\left(\left(a_{11}^{l}\right)^{\lambda},\left(a_{12}^{l}\right)^{\lambda},\left(a_{13}^{l}\right)^{\lambda},\left(a_{14}^{l}\right)^{\lambda} ;\left(h_{1}\left(A_{1}^{l}\right)\right)^{\lambda},\left(h_{2}\left(A_{1}^{l}\right)\right)^{\lambda}\right)\right) .
\end{aligned}
$$

(2) For Einstein T-norm $T_{E}(x, y)$, Eqs. (6)-(7) become $(\lambda>0)$ :

$$
\begin{aligned}
& A_{1} \otimes A_{2}=\left(\left(\frac{a_{11}^{u} a_{21}^{u}}{1+\left(1-a_{11}^{u}\right)\left(1-a_{21}^{u}\right)}, \frac{a_{12}^{u} a_{22}^{u}}{1+\left(1-a_{12}^{u}\right)\left(1-a_{22}^{u}\right)}, \frac{a_{13}^{u} a_{23}^{u}}{1+\left(1-a_{13}^{u}\right)\left(1-a_{23}^{u}\right)}, \frac{a_{14}^{u} a_{24}^{u}}{1+\left(1-a_{14}^{u}\right)\left(1-a_{24}^{u}\right)} ;\right.\right. \\
& \left.\frac{h_{1}\left(A_{1}^{u}\right) h_{1}\left(A_{2}^{u}\right)}{1+\left(1-h_{1}\left(A_{1}^{u}\right)\right)\left(1-h_{1}\left(A_{2}^{u}\right)\right)}, \frac{h_{2}\left(A_{1}^{u}\right) h_{2}\left(A_{2}^{u}\right)}{1+\left(1-h_{2}\left(A_{1}^{u}\right)\right)\left(1-h_{2}\left(A_{2}^{u}\right)\right)}\right) \text {, } \\
& \left(\frac{a_{11}^{l} a_{21}^{l}}{1+\left(1-a_{11}^{l}\right)\left(1-a_{21}^{l}\right)}, \frac{a_{12}^{l} a_{22}^{l}}{1+\left(1-a_{12}^{l}\right)\left(1-a_{22}^{l}\right)}, \frac{a_{13}^{l} a_{23}^{l}}{1+\left(1-a_{13}^{l}\right)\left(1-a_{23}^{l}\right)}, \frac{a_{14}^{l} a_{24}^{l}}{1+\left(1-a_{14}^{l}\right)\left(1-a_{24}^{l}\right)}\right. \text {; } \\
& \left.\left.\frac{h_{1}\left(A_{1}^{l}\right) h_{1}\left(A_{2}^{l}\right)}{1+\left(1-h_{1}\left(A_{1}^{l}\right)\right)\left(1-h_{1}\left(A_{2}^{l}\right)\right)}, \frac{h_{2}\left(A_{1}^{l}\right) h_{2}\left(A_{2}^{l}\right)}{1+\left(1-h_{2}\left(A_{1}^{l}\right)\right)\left(1-h_{2}\left(A_{2}^{l}\right)\right)}\right)\right) \text {, } \\
& \left(A_{1}\right)^{\lambda}=\left(\left(\frac{2\left(a_{11}^{u}\right)^{\lambda}}{\left(2-a_{11}^{u}\right)^{\lambda}+\left(a_{11}^{u}\right)^{\lambda}}, \frac{2\left(a_{12}^{u}\right)^{\lambda}}{\left(2-a_{12}^{u}\right)^{\lambda}+\left(a_{12}^{u}\right)^{\lambda}}, \frac{2\left(a_{13}^{u}\right)^{\lambda}}{\left(2-a_{13}^{u}\right)^{\lambda}+\left(a_{13}^{u}\right)^{\lambda}}, \frac{2\left(a_{14}^{u}\right)^{\lambda}}{\left(2-a_{14}^{u}\right)^{\lambda}+\left(a_{14}^{u}\right)^{\lambda}} ;\right.\right. \\
& \left.\frac{2\left(h_{1}\left(A_{1}^{u}\right)\right)^{\lambda}}{\left(2-h_{1}\left(A_{1}^{u}\right)\right)^{\lambda}+\left(h_{1}\left(A_{1}^{u}\right)\right)^{\lambda}}, \frac{2\left(h_{2}\left(A_{1}^{u}\right)\right)^{\lambda}}{\left(2-h_{2}\left(A_{1}^{u}\right)\right)^{\lambda}+\left(h_{2}\left(A_{1}^{u}\right)\right)^{\lambda}}\right), \\
& \left(\frac{2\left(a_{11}^{l}\right)^{\lambda}}{\left(2-a_{12}^{l}\right)^{\lambda}+\left(a_{11}^{l}\right)^{\lambda}}, \frac{2\left(a_{12}^{l}\right)^{\lambda}}{\left(2-a_{12}^{l}\right)^{\lambda}+\left(a_{12}^{l}\right)^{\lambda}}, \frac{2\left(a_{13}^{l}\right)^{\lambda}}{\left(2-a_{13}^{l}\right)^{\lambda}+\left(a_{13}^{l}\right)^{\lambda}}, \frac{2\left(a_{14}^{l}\right)^{\lambda}}{\left(2-a_{14}^{l}\right)^{\lambda}+\left(a_{14}^{l}\right)^{\lambda}}\right. \text {; } \\
& \left.\left.\frac{2\left(h_{1}\left(A_{1}^{l}\right)\right)^{\lambda}}{\left(2-h_{1}\left(A_{1}^{l}\right)\right)^{\lambda}+\left(h_{1}\left(A_{1}^{l}\right)\right)^{\lambda}}, \frac{2\left(h_{2}\left(A_{1}^{l}\right)\right)^{\lambda}}{\left(2-h_{2}\left(A_{1}^{l}\right)\right)^{\lambda}+\left(h_{2}\left(A_{1}^{l}\right)\right)^{\lambda}}\right)\right) \text {. }
\end{aligned}
$$

(3) For the Frank T-norm $T_{F}(x, y)$, Eqs. (6)-(7) become $(\lambda>0, \gamma>0$ and $\gamma \neq 1)$ :

$$
\begin{aligned}
& A_{1} \otimes A_{2}=\left(\left(\log _{\gamma}\left(1+\frac{\prod_{k=1}^{2}\left(\gamma^{a_{k 1}^{u}}-1\right)}{\gamma-1}\right), \log _{\gamma}\left(1+\frac{\prod_{\kappa=1}^{2}\left(\gamma^{a_{k 2}^{u}}-1\right)}{\gamma-1}\right), \log _{\gamma}\left(1+\frac{\prod_{\kappa=1}^{2}\left(\gamma^{a_{k 3}^{u}}-1\right)}{\gamma-1}\right),\right.\right. \\
& \left.\log _{\gamma}\left(1+\frac{\prod_{\kappa=1}^{2}\left(\gamma^{a_{k 4}^{u}}-1\right)}{\gamma-1}\right) ; \log _{\gamma}\left(1+\frac{\prod_{\kappa=1}^{2}\left(\gamma^{h_{1}\left(A_{k}^{u}\right)}-1\right)}{\gamma-1}\right), \log _{\gamma}\left(1+\frac{\prod_{k=1}^{2}\left(\gamma^{h_{2}\left(A_{k}^{u}\right)}-1\right)}{\gamma-1}\right)\right), \\
& \left(\log _{\gamma}\left(1+\frac{\prod_{\kappa=1}^{2}\left(\gamma^{a_{k 1}^{l}}-1\right)}{\gamma-1}\right), \log _{\gamma}\left(1+\frac{\prod_{\kappa=1}^{2}\left(\gamma^{a_{k 2}^{l}}-1\right)}{\gamma-1}\right), \log _{\gamma}\left(1+\frac{\prod_{\kappa=1}^{2}\left(\gamma^{a_{k 3}^{l}}-1\right)}{\gamma-1}\right),\right. \\
& \left.\log _{\gamma}\left(1+\frac{\prod_{\kappa=1}^{2}\left(\gamma^{a_{k 4}^{l}}-1\right)}{\gamma-1}\right) ; \log _{\gamma}\left(1+\frac{\prod_{\kappa=1}^{2}\left(\gamma^{h_{1}\left(A_{\kappa}^{l}\right)}-1\right)}{\gamma-1}\right), \log _{\gamma}\left(1+\frac{\prod_{\kappa=1}^{2}\left(\gamma^{h_{2}\left(A_{\kappa}^{l}\right)}-1\right)}{\gamma-1}\right)\right), \\
& \left(A_{1}\right)^{\lambda}=\left(\left(\log _{\gamma}\left(1+\frac{\left(\gamma^{a_{11}^{u}}-1\right)^{\lambda}}{(\gamma-1)^{\lambda-1}}\right), \log _{\gamma}\left(1+\frac{\left(\gamma^{a_{12}^{u}}-1\right)^{\lambda}}{(\gamma-1)^{\lambda-1}}\right), \log _{\gamma}\left(1+\frac{\left(\gamma^{a_{13}^{u}}-1\right)^{\lambda}}{(\gamma-1)^{\lambda-1}}\right), \log _{\gamma}\left(1+\frac{\left(\gamma^{a_{14}^{u}}-1\right)^{\lambda}}{(\gamma-1)^{\lambda-1}}\right) ;\right.\right. \\
& \left.\log _{\gamma}\left(1+\frac{\left(\gamma^{h_{1}\left(A_{1}^{u}\right)}-1\right)^{\lambda}}{(\gamma-1)^{\lambda-1}}\right), \log _{\gamma}\left(1+\frac{\left(\gamma^{h_{2}\left(A_{1}^{u}\right)}-1\right)^{\lambda}}{(\gamma-1)^{\lambda-1}}\right)\right),\left(\log _{\gamma}\left(1+\frac{\left(\gamma^{a_{11}^{l}}-1\right)^{\lambda}}{(\gamma-1)^{\lambda-1}}\right), \log _{\gamma}\left(1+\frac{\left(\gamma^{a_{12}^{\prime}}-1\right)^{\lambda}}{(\gamma-1)^{\lambda-1}}\right),\right. \\
& \left.\left.\log _{\gamma}\left(1+\frac{\left(\gamma^{a_{13}^{\prime}}-1\right)^{\lambda}}{(\gamma-1)^{\lambda-1}}\right), \log _{\gamma}\left(1+\frac{\left(\gamma^{a_{14}^{l}}-1\right)^{\lambda}}{(\gamma-1)^{\lambda-1}}\right) ; \log _{\gamma}\left(1+\frac{\left(\gamma^{h_{1}\left(A_{1}^{\prime}\right)}-1\right)^{\lambda}}{(\gamma-1)^{\lambda-1}}\right), \log _{\gamma}\left(1+\frac{\left(\gamma^{h_{2}\left(A_{1}^{\prime}\right)}-1\right)^{\lambda}}{(\gamma-1)^{\lambda-1}}\right)\right)\right) \text {. }
\end{aligned}
$$

When $\quad a_{12}^{u}=a_{13}^{u}=a_{12}^{l}=a_{13}^{l}=a_{22}^{u}=a_{23}^{u}=a_{22}^{l}=a_{23}^{l}, \quad h_{1}\left(A_{1}^{u}\right)=h_{2}\left(A_{1}^{u}\right)=h_{1}\left(A_{1}^{l}\right)=h_{2}\left(A_{1}^{l}\right)=1 \quad$ and 
$h_{1}\left(A_{2}^{u}\right)=h_{2}\left(A_{2}^{u}\right)=h_{1}\left(A_{2}^{l}\right)=h_{2}\left(A_{2}^{l}\right)=1$, Eqs. (12)-(13) become the operations defined by Qin and Liu [33].

(4) For the Aczel-Alsina T-norm $T_{A A}(x, y)$, Eqs. (6)-(7) become $(\lambda>0$ and $\gamma>0)$ :

$$
\begin{aligned}
& A_{1} \otimes A_{2}=\left(\left(e^{-\left(\left(-\ln a_{11}^{u}\right)^{\gamma}+\left(-\ln a_{21}^{u}\right)^{\gamma}\right)^{1 / \gamma}}, e^{-\left(\left(-\ln a_{12}^{u}\right)^{\gamma}+\left(-\ln a_{22}^{u}\right)^{\gamma}\right)^{1 / \gamma}}, e^{-\left(\left(-\ln a_{13}^{u}\right)^{\gamma}+\left(-\ln a_{23}^{u} \gamma^{\gamma}\right)^{1 / \gamma}\right.}, e^{-\left(\left(-\ln a_{14}^{u}\right)^{\gamma}+\left(-\ln a_{24}^{u}\right)^{\gamma}\right)^{1 / \gamma}} ;\right.\right. \\
& \left.e^{-\left(\left(-\ln h_{1}\left(A_{1}^{u}\right)\right)^{\gamma}+\left(-\ln h_{1}\left(A_{2}^{u}\right)\right)^{\gamma}\right)^{1 / \gamma}} e^{-\left(\left(-\ln h_{2}\left(A_{1}^{u}\right)\right)^{\gamma}+\left(-\ln h_{2}\left(A_{2}^{u}\right)\right)^{\gamma}\right)^{1 / \gamma}}\right),\left(e^{-\left(\left(-\ln a_{11}^{l}\right)^{\gamma}+\left(-\ln \left(a_{21}^{l}\right)\right)^{\gamma}\right)^{1 / \gamma}}, e^{-\left(\left(-\ln a_{12}^{l} \gamma^{\gamma}+\left(-\ln a_{22}^{l}\right)^{\gamma}\right)^{1 / \gamma}\right.},\right. \\
& \left.\left.e^{-\left(\left(-\ln a_{13}^{l}\right)^{\gamma}+\left(-\ln a_{23}^{l}\right)^{\gamma}\right)^{1 / \gamma}}, e^{-\left(\left(-\ln a_{14}^{l}\right)^{\gamma}+\left(-\ln a_{24}^{l}\right)^{\gamma}\right)^{1 / \gamma}} ; e^{-\left(\left(-\ln h_{1}\left(A_{1}^{l}\right)\right)^{\gamma}+\left(-\ln h_{1}\left(A_{2}^{l}\right)\right)^{\gamma}\right)^{1 / \gamma}}, e^{-\left(\left(-\ln h_{2}\left(A_{1}^{l}\right)\right)^{\gamma}+\left(-\ln h_{2}\left(A_{2}^{l}\right)\right)^{\gamma}\right)^{1 / \gamma}}\right)\right), \\
& \left(A_{1}\right)^{\lambda}=\left(\left(e^{-\left(\lambda\left(-\ln a_{11}^{u}\right)^{\gamma}\right)^{1 / \gamma}}, e^{-\left(\lambda\left(-\ln a_{12}^{u}\right)^{\gamma}\right)^{1 / \gamma}}, e^{-\left(\lambda\left(-\ln a_{13}^{u}\right)^{\gamma}\right)^{1 / \gamma}}, e^{-\left(\lambda\left(-\ln a_{14}^{u}\right)^{\gamma}\right)^{1 / \gamma}} ; e^{-\left(\lambda\left(-\ln h_{1}\left(A_{1}^{u}\right)^{\gamma}\right)^{1 / \gamma}\right.} e^{-\left(\lambda\left(-\ln h_{2}\left(A_{1}^{u}\right)\right)^{\gamma}\right)^{1 / \gamma}}\right),\right. \\
& \left.\left(e^{-\left(\lambda\left(-\ln a_{11}^{l}\right)^{\gamma}\right)^{1 / \gamma}}, e^{-\left(\lambda\left(-\ln a_{12}^{l}\right)^{\gamma}\right)^{1 / \gamma}}, e^{-\left(\lambda\left(-\ln a_{13}^{l}\right)^{\gamma}\right)^{1 / \gamma}}, e^{-\left(\lambda\left(-\ln a_{14}^{l}\right)^{\gamma}\right)^{1 / \gamma}} ; e^{-\left(\lambda\left(-\ln h_{1}\left(A_{1}^{l}\right)^{\gamma}\right)^{1 / \gamma}\right.}, e^{-\left(\lambda\left(-\ln h_{2}\left(A_{1}^{l}\right)\right)^{\gamma}\right)^{1 / \gamma}}\right)\right) .
\end{aligned}
$$

\subsection{Fuzzy measure and Choquet integral operator}

A FM [37] on the set $Z=\left\{z_{1}, z_{2}, \ldots, z_{n}\right\}$ is a function $\mu: P(Z) \rightarrow[0,1]$, where $P(Z)$ denotes the power set of $Z$, which satisfies the following two restrictions:

(1) Boundedness: $\mu(\phi)=0, \mu(Z)=1$;

(2) Monotonicity: If $B, D \in P(Z)$ and $D \subset B$, then $\mu(D) \leq \mu(B)$.

The value $\mu(D)$ can be considered as the grade of importance of the set $D$, where $D \in P(Z)$. Thus, in addition to the traditional weight on a single element $z_{i} \in Z$, weights on any coalition of elements $z_{i} \in Z$ are also defined with an FM $\mu$.

FMs can be utilized for fusion purposes in conjunction with the Choquet integral.

Definition 6 [37]. Let $\mu$ be an FM on the set $Z$ and $f$ a function on the set $Z$. The Choquet integral of $f$ is:

$$
C_{\mu}\left(f\left(z_{(1)}\right), f\left(z_{(2)}\right), \ldots, f\left(z_{(n)}\right)\right)=\sum_{i=1}^{n} f\left(z_{(i)}\right)\left(\mu\left(Z_{(i)}\right)-\mu\left(Z_{(i+1)}\right)\right)
$$

where the subscript (.) represents the permutation of the elements in the set $Z$ such that $f\left(z_{(1)}\right) \leq f\left(z_{(2)}\right) \leq \ldots \leq f\left(z_{(n)}\right)$, and $Z_{(i)}=\left\{z_{(i)}, z_{(i+1)}, \ldots, z_{(n)}\right\}$ with $Z_{(n+1)}=\phi \quad(i=1,2, \ldots, n)$.

\section{The Choquet integral of IT2FNs based on Archimedean T-norms}

In this section, we propose the IT2FAC operator, the BIT2FAC operator and the 2ABIT2FAC operator for aggregating IT2FNs. Then, several features and special cases of these novel operators are studied.

\subsection{The IT2FAC operator}

Definition 7. It is assumed that $Z=\left\{z_{1}, z_{2}, \ldots, z_{n}\right\}$ is a collection of attributes and $\Lambda=\left\{A_{1}, A_{2}, \ldots, A_{n}\right\}$ is a set of trapezoidal IT2FNs on $Z$. The IT2FAC operator is expressed as follows: 


$$
\operatorname{IT} 2 \operatorname{FAC}\left(A_{1}, A_{2}, \ldots, A_{n}\right)=\bigotimes_{k=1}^{n} A_{(k)}^{\mu\left(Z_{(k)}\right)-\mu\left(Z_{(k+1)}\right)},
$$

where $A_{(k)}$ denotes the $k$-th smallest element in the set $\Lambda=\left\{A_{1}, A_{2}, \ldots, A_{n}\right\}, z_{(k)}$ is the attribute corresponding to $A_{(k)}, \quad Z_{(k)}=\left\{z_{(k)}, z_{(k+1)}, \ldots, z_{(n)}\right\}$ with $Z_{(n+1)}=\phi$, and $\mu$ represents an FM on $Z$.

Based on the operations of IT2FNs listed in Eqs. (6)-(7), from Eq. (16), we can derive the following result.

Theorem 1. The aggregation output of the set $\Lambda=\left\{A_{1}, A_{2}, \ldots, A_{n}\right\}$ of trapezoidal IT2FNs by the IT2FAC operator is the following trapezoidal IT2FN:

$$
\begin{array}{r}
\operatorname{IT2FAC}\left(A_{1}, A_{2}, \ldots, A_{n}\right) \\
=\left(\left(g^{-1}\left(\sum_{k=1}^{n}\left(\mu\left(Z_{(k)}\right)-\mu\left(Z_{(k+1)}\right)\right) g\left(a_{(k) 1}^{u}\right)\right), g^{-1}\left(\sum_{k=1}^{n}\left(\mu\left(Z_{(k)}\right)-\mu\left(Z_{(k+1)}\right)\right) g\left(a_{(k) 2}^{u}\right)\right),\right.\right. \\
g^{-1}\left(\sum_{k=1}^{n}\left(\mu\left(Z_{(k)}\right)-\mu\left(Z_{(k+1)}\right)\right) g\left(a_{(k) 3}^{u}\right)\right), g^{-1}\left(\sum_{k=1}^{n}\left(\mu\left(Z_{(k)}\right)-\mu\left(Z_{(k+1)}\right)\right) g\left(a_{(k) 4}^{u}\right)\right) ; \\
\left.g^{-1}\left(\sum_{k=1}^{n}\left(\mu\left(Z_{(k)}\right)-\mu\left(Z_{(k+1)}\right)\right) g\left(h_{1}\left(A_{(k)}^{u}\right)\right)\right), g^{-1}\left(\sum_{k=1}^{n}\left(\mu\left(Z_{(k)}\right)-\mu\left(Z_{(k+1)}\right)\right) g\left(h_{2}\left(A_{(k)}^{u}\right)\right)\right)\right), \\
\quad\left(g^{-1}\left(\sum_{k=1}^{n}\left(\mu\left(Z_{(k)}\right)-\mu\left(Z_{(k+1)}\right)\right) g\left(a_{(k) 1}^{l}\right)\right), g^{-1}\left(\sum_{k=1}^{n}\left(\mu\left(Z_{(k)}\right)-\mu\left(Z_{(k+1)}\right)\right) g\left(a_{(k) 2}^{l}\right)\right),\right. \\
g^{-1}\left(\sum_{k=1}^{n}\left(\mu\left(Z_{(k)}\right)-\mu\left(Z_{(k+1)}\right)\right) g\left(a_{(k) 3}^{l}\right)\right), g^{-1}\left(\sum_{k=1}^{n}\left(\mu\left(Z_{(k)}\right)-\mu\left(Z_{(k+1)}\right)\right) g\left(a_{(k) 4}^{l}\right)\right) ; \\
\left.\left.g^{-1}\left(\sum_{k=1}^{n}\left(\mu\left(Z_{(k)}\right)-\mu\left(Z_{(k+1)}\right)\right) g\left(h_{1}\left(A_{(k)}^{l}\right)\right)\right), g^{-1}\left(\sum_{k=1}^{n}\left(\mu\left(Z_{(k)}\right)-\mu\left(Z_{(k+1)}\right)\right) g\left(h_{2}\left(A_{(k)}^{l}\right)\right)\right)\right)\right) .
\end{array}
$$

The proof of Theorem 1 is provided in the supplementary material Appendix 1.

Corollary 1. When all elements $z_{k}(k=1,2, \ldots, n)$ in the set $Z$ are independent, the FM $\mu$ is an additive measure, i.e., $\mu(D)=\sum_{z_{k} \in D} \mu\left(\left\{z_{k}\right\}\right)$ for any $D \subset Z$, and the IT2FAC operator becomes the following IT2F Archimedean weighted average (IT2FAWA) operator:

$$
\begin{aligned}
& I T 2 F A W A\left(A_{1}, A_{2}, \ldots, A_{n}\right) \\
= & \left(\left(g^{-1}\left(\sum_{k=1}^{n} \mu\left(\left\{z_{k}\right\}\right) g\left(a_{k 1}^{u}\right)\right), g^{-1}\left(\sum_{k=1}^{n} \mu\left(\left\{z_{k}\right\}\right) g\left(a_{k 2}^{u}\right)\right), g^{-1}\left(\sum_{k=1}^{n} \mu\left(\left\{z_{k}\right\}\right) g\left(a_{k 3}^{u}\right)\right),\right.\right. \\
& \left.g^{-1}\left(\sum_{k=1}^{n} \mu\left(\left\{z_{k}\right\}\right) g\left(a_{k 4}^{u}\right)\right) ; g^{-1}\left(\sum_{k=1}^{n} \mu\left(\left\{z_{k}\right\}\right) g\left(h_{1}\left(A_{k}^{u}\right)\right)\right), g^{-1}\left(\sum_{k=1}^{n} \mu\left(\left\{z_{k}\right\}\right) g\left(h_{2}\left(A_{k}^{u}\right)\right)\right)\right), \\
& \left(g^{-1}\left(\sum_{k=1}^{n} \mu\left(\left\{z_{k}\right\}\right) g\left(a_{k 1}^{l}\right)\right), g^{-1}\left(\sum_{k=1}^{n} \mu\left(\left\{z_{k}\right\}\right) g\left(a_{k 2}^{l}\right)\right), g^{-1}\left(\sum_{k=1}^{n} \mu\left(\left\{z_{k}\right\}\right) g\left(a_{k 3}^{l}\right)\right),\right. \\
& \left.\left.g^{-1}\left(\sum_{k=1}^{n} \mu\left(\left\{z_{k}\right\}\right) g\left(a_{k 4}^{l}\right)\right) ; g^{-1}\left(\sum_{k=1}^{n} \mu\left(\left\{z_{k}\right\}\right) g\left(h_{1}\left(A_{k}^{l}\right)\right)\right), g^{-1}\left(\sum_{k=1}^{n} \mu\left(\left\{z_{k}\right\}\right) g\left(h_{2}\left(A_{k}^{l}\right)\right)\right)\right)\right) .
\end{aligned}
$$

Corollary 2. If $\mu(D)=\sum_{k=1}^{|D|} w_{k}$ for any $D \subset Z$, where $|D|$ represents the cardinality of $D \subset Z$, then the IT2FAC operator becomes the following IT2F Archimedean ordered weighted average (IT2FAOWA) operator: 


$$
\begin{aligned}
& \operatorname{IT} 2 \text { FAOWA }\left(A_{1}, A_{2}, \ldots, A_{n}\right) \\
= & \left(\left(g^{-1}\left(\sum_{k=1}^{n} w_{k} g\left(a_{(k) 1}^{u}\right)\right), g^{-1}\left(\sum_{k=1}^{n} w_{k} g\left(a_{(k) 2}^{u}\right)\right), g^{-1}\left(\sum_{k=1}^{n} w_{k} g\left(a_{(k) 3}^{u}\right)\right), g^{-1}\left(\sum_{k=1}^{n} w_{k} g\left(a_{(k) 4}^{u}\right)\right) ;\right.\right. \\
& g^{-1}\left(\sum_{k=1}^{n} w_{k} g\left(h_{1}\left(A_{(k)}^{u}\right)\right)\right), g^{-1}\left(\sum_{k=1}^{n} w_{k} g\left(h_{2}\left(A_{(k)}^{u}\right)\right)\right),\left(g^{-1}\left(\sum_{k=1}^{n} w_{k} g\left(a_{(k) 1}^{l}\right)\right), g^{-1}\left(\sum_{k=1}^{n} w_{k} g\left(a_{(k) 2}^{l}\right)\right),\right. \\
& \left.\left.g^{-1}\left(\sum_{k=1}^{n} w_{k} g\left(a_{(k) 3}^{l}\right)\right), g^{-1}\left(\sum_{k=1}^{n} w_{k} g\left(a_{(k) 4}^{l}\right)\right) ; g^{-1}\left(\sum_{k=1}^{n} w_{k} g\left(h_{1}\left(A_{(k)}^{l}\right)\right)\right), g^{-1}\left(\sum_{k=1}^{n} w_{k} g\left(h_{2}\left(A_{(k)}^{l}\right)\right)\right)\right)\right) .
\end{aligned}
$$

In what follows, some fundamental features of the proposed IT2FAC operator are investigated.

Theorem 2. Let $\Lambda=\left\{A_{1}, A_{2}, \ldots, A_{n}\right\}$ and $\Lambda^{\prime}=\left\{A_{1}^{\prime}, A_{2}^{\prime}, \ldots, A_{n}^{\prime}\right\}$ be two sets of trapezoidal IT2FNs on $Z$, $B$ a trapezoidal IT2FN and $\xi>0$. The IT2FAC operator verifies the following properties:

(1) Idempotency: IT $2 F A C(A, A, \ldots, A)=A$, where $A=A_{k}(k=1,2, \ldots, n)$.

(2) Commutativity: IT2FAC $\left(A_{1}^{\prime}, A_{2}^{\prime}, \ldots, A_{n}^{\prime}\right)=\operatorname{IT} 2 F A C\left(A_{1}, A_{2}, \ldots, A_{n}\right)$, where $\left\{A_{1}^{\prime}, A_{2}^{\prime}, \ldots, A_{n}^{\prime}\right\}$ is any permutation of $\left\{A_{1}, A_{2}, \ldots, A_{n}\right\}$.

(3) Monotonicity: When $a_{(k) 1}^{\prime u} \leq a_{(k) 1}^{u}, a_{(k) 2}^{\prime u} \leq a_{(k) 2}^{u}, a_{(k) 3}^{\prime u} \leq a_{(k) 3}^{u}, a_{(k) 4}^{\prime u} \leq a_{(k) 4}^{u}, h_{1}^{u}\left(A_{(k)}^{\prime}\right) \leq h_{1}^{u}\left(A_{(k)}\right)$, $h_{2}^{u}\left(A_{(k)}^{\prime}\right) \leq h_{2}^{u}\left(A_{(k)}\right), \quad a_{(k) 1}^{\prime l} \leq a_{(k) 1}^{l}, \quad a_{(k) 2}^{\prime l} \leq a_{(k) 2}^{l}, \quad a_{(k) 3}^{\prime l} \leq a_{(k) 3}^{l}, \quad a_{(k) 4}^{\prime l} \leq a_{(k) 4}^{l}, \quad h_{1}^{l}\left(A_{(k)}^{\prime}\right) \leq h_{1}^{l}\left(A_{(k)}\right)$, $h_{2}^{l}\left(A_{(k)}^{\prime}\right) \leq h_{2}^{l}\left(A_{(k)}\right)$, it is $\operatorname{IT} 2 F A C\left(A_{1}^{\prime}, A_{2}^{\prime}, \ldots, A_{n}^{\prime}\right) \leq \operatorname{IT} 2 F A C\left(A_{1}, A_{2}, \ldots, A_{n}\right)$, where $A_{(k)}^{\prime}$ is the $k$-th smallest value of the set $\Lambda^{\prime}$.

(4) Boundedness: $A^{-} \leq \operatorname{IT} 2 F A C\left(A_{1}, A_{2}, \ldots, A_{n}\right) \leq A^{+}$, where

$$
\begin{aligned}
A^{-}= & \left(\left(\min _{k}\left(a_{k 1}^{u}\right), \min _{k}\left(a_{k 2}^{u}\right), \min _{k}\left(a_{k 3}^{u}\right), \min _{k}\left(a_{k 4}^{u}\right) ; \min _{k}\left(h_{1}\left(A_{k}^{u}\right)\right), \min _{k}\left(h_{2}\left(A_{k}^{u}\right)\right)\right),\right. \\
& \left.\left(\min _{k}\left(a_{k 1}^{l}\right), \min _{k}\left(a_{k 2}^{l}\right), \min _{k}\left(a_{k 3}^{l}\right), \min _{k}\left(a_{k 4}^{l}\right) ; \min _{k}\left(h_{1}\left(A_{k}^{l}\right)\right), \min _{k}\left(h_{2}\left(A_{k}^{l}\right)\right)\right)\right)
\end{aligned}
$$

and

$$
\begin{aligned}
& A^{+}=\left(\left(\max _{k}\left(a_{k 1}^{u}\right), \max _{k}\left(a_{k 2}^{u}\right), \max _{k}\left(a_{k 3}^{u}\right), \max _{k}\left(a_{k 4}^{u}\right) ; \max _{k}\left(h_{1}\left(A_{k}^{u}\right)\right), \max _{k}\left(h_{2}\left(A_{k}^{u}\right)\right)\right),\right. \\
&\left.\left(\max _{k}\left(a_{k 1}^{l}\right), \max _{k}\left(a_{k 2}^{l}\right), \max _{k}\left(a_{k 3}^{l}\right), \max _{k}\left(a_{k 4}^{l}\right) ; \max _{k}\left(h_{1}\left(A_{k}^{l}\right)\right), \max _{k}\left(h_{2}\left(A_{k}^{l}\right)\right)\right)\right) . \\
& \text { (5) } \operatorname{IT} 2 F A C\left(A_{1}^{\xi} \otimes B, A_{2}^{\xi} \otimes B, \ldots, A_{n}^{\xi} \otimes B\right)=\left(\operatorname{IT} 2 F A C\left(A_{1}, A_{2}, \ldots, A_{n}\right)\right)^{\xi} \otimes B . \\
& \text { (6) IT } 2 F A C\left(A_{1} \otimes A_{1}^{\prime}, A_{2} \otimes A_{2}^{\prime}, \ldots, A_{n} \otimes A_{n}^{\prime}\right)=\operatorname{IT} 2 F A C\left(A_{1}, A_{2}, \ldots, A_{n}\right) \otimes I T 2 F A C\left(A_{1}^{\prime}, A_{2}^{\prime}, \ldots, A_{n}^{\prime}\right) .
\end{aligned}
$$

The proof of Theorem 2 is provided in the supplementary material Appendix 2.

In what follows, we derive the special cases of the proposed IT2FAC operator when using the different additive generator functions of Table 1 .

(1) When we utilize the algebraic operations, the IT2FAC operator becomes the IT2F Choquet (IT2FC) operator, i.e.: 


$$
\begin{gathered}
\operatorname{IT2FC}\left(A_{1}, A_{2}, \ldots, A_{n}\right) \\
=\left(\left(\prod_{k=1}^{n}\left(a_{(k) 1}^{u}\right)^{\mu\left(Z_{(k)}\right)-\mu\left(Z_{(k+1)}\right)}, \prod_{k=1}^{n}\left(a_{(k) 2}^{u}\right)^{\mu\left(Z_{(k)}\right)-\mu\left(Z_{(k+1)}\right)}, \prod_{k=1}^{n}\left(a_{(k) 3}^{u}\right)^{\mu\left(Z_{(k)}\right)-\mu\left(Z_{(k+1)}\right)}, \prod_{k=1}^{n}\left(a_{(k) 4}^{u}\right)^{\mu\left(Z_{(k)}\right)-\mu\left(Z_{(k+1)}\right)} ;\right.\right. \\
\left.\prod_{k=1}^{n}\left(h_{1}\left(A_{(k)}^{u}\right)\right)^{\mu\left(Z_{(k)}\right)-\mu\left(Z_{(k+1)}\right)}, \prod_{k=1}^{n}\left(h_{2}\left(A_{(k)}^{u}\right)\right)^{\mu\left(Z_{(k)}\right)-\mu\left(Z_{(k+1)}\right)}\right), \\
\left(\prod_{k=1}^{n}\left(a_{(k) 1}^{l}\right)^{\mu\left(Z_{(k)}\right)-\mu\left(Z_{(k+1)}\right)}, \prod_{k=1}^{n}\left(a_{(k) 2}^{l}\right)^{\mu\left(Z_{(k)}\right)-\mu\left(Z_{(k+1)}\right)}, \prod_{k=1}^{n}\left(a_{(k) 3}^{l}\right)^{\mu\left(Z_{(k)}\right)-\mu\left(Z_{(k+1)}\right)}, \prod_{k=1}^{n}\left(a_{(k) 4}^{l}\right)^{\mu\left(Z_{(k)}\right)-\mu\left(Z_{(k+1)}\right)} ;\right. \\
\left.\left.\prod_{k=1}^{n}\left(h_{1}\left(A_{(k)}^{l}\right)\right)^{\mu\left(Z_{(k)}\right)-\mu\left(Z_{(k+1)}\right)}, \prod_{k=1}^{n}\left(h_{2}\left(A_{(k)}^{l}\right)\right)^{\mu\left(Z_{(k)}\right)-\mu\left(Z_{(k+1)}\right)}\right)\right) .
\end{gathered}
$$

(2) When we utilize the Einstein operations, the IT2FAC operator becomes the Einstein IT2FC (EIT2FC) operator, i.e.:

$$
\begin{aligned}
& \operatorname{EIT} 2 F C\left(A_{1}, A_{2}, \ldots, A_{n}\right)= \\
& \int\left(\int_{k=1}^{n} \frac{2 \prod_{k=1}^{n}\left(a_{(k) 1}^{u}\right)^{\mu\left(Z_{(k)}\right)-\mu\left(Z_{(k+1)}\right)}}{\prod_{k}^{n}\left(2-a_{(k) 1}^{u}\right)^{\mu\left(Z_{(k)}\right)-\mu\left(Z_{(k+1)}\right)}+\prod_{k=1}^{n}\left(a_{(k) 1}^{u}\right)^{\mu\left(Z_{(k)}\right)-\mu\left(Z_{(k+1)}\right)}}, \frac{2 \prod_{k=1}^{n}\left(a_{(k) 2}^{u}\right)^{\mu\left(Z_{(k)}\right)-\mu\left(Z_{(k+1)}\right)}}{\prod_{k=1}^{n}\left(2-a_{(k) 2}^{u}\right)^{\mu\left(Z_{(k)}\right)-\mu\left(Z_{(k+1)}\right)}+\prod_{k=1}^{n}\left(a_{(k) 2}^{u}\right)^{\mu\left(Z_{(k)}\right)-\mu\left(Z_{(k+1)}\right)}},\right. \\
& \frac{2 \prod_{k=1}^{n}\left(a_{(k) 3}^{u}\right)^{\mu\left(Z_{(k)}\right)-\mu\left(Z_{(k+1)}\right)}}{\prod_{k=1}^{n}\left(2-a_{(k) 3}^{u}\right)^{\mu\left(Z_{(k)}\right)-\mu\left(Z_{(k+1)}\right)}+\prod_{k=1}^{n}\left(a_{(k) 3}^{u}\right)^{\mu\left(Z_{(k)}\right)-\mu\left(Z_{(k+1)}\right)}}, \frac{2 \prod_{k=1}^{n}\left(a_{(k) 4}^{u}\right)^{\mu\left(Z_{(k)}\right)-\mu\left(Z_{(k+1)}\right)}}{\prod_{k=1}^{n}\left(2-a_{(k) 4}^{u}\right)^{\mu\left(Z_{(k)}\right)-\mu\left(Z_{(k+1)}\right)}+\prod_{k=1}^{n}\left(a_{(k) 4}^{u}\right)^{\mu\left(Z_{(k)}\right)-\mu\left(Z_{(k+1)}\right)}} ; \\
& \left.\frac{2 \prod_{k=1}^{n}\left(h_{1}\left(A_{(k)}^{u}\right)\right)^{\mu\left(Z_{(k)}\right)-\mu\left(Z_{(k+1)}\right)}}{\prod_{k=1}^{n}\left(2-h_{1}\left(A_{(k)}^{u}\right)\right)^{\mu\left(Z_{(k)}\right)-\mu\left(Z_{(k+1)}\right)}+\prod_{k=1}^{n}\left(h_{1}\left(A_{(k)}^{u}\right)\right)^{\mu\left(Z_{(k)}\right)-\mu\left(Z_{(k+1)}\right)}}, \frac{2 \prod_{k=1}^{n}\left(h_{2}\left(A_{(k)}^{u}\right)\right)^{\mu\left(Z_{(k)}\right)-\mu\left(Z_{(k+1)}\right)}}{\left.\prod_{k=1}^{n}\left(2-h_{2}\left(A_{(k)}^{u}\right)\right)^{\mu\left(Z_{(k)}\right)-\mu\left(Z_{(k+1)}\right)}+\prod_{k=1}^{n}\left(h_{2}\left(A_{(k)}^{u}\right)\right)^{\mu\left(Z_{(k)}\right)-\mu\left(Z_{(k+1)}\right)}\right)}\right), \\
& \int \frac{2 \prod_{k=1}^{n}\left(a_{(k) 1}^{l}\right)^{\mu\left(Z_{(k)}\right)-\mu\left(Z_{(k+1)}\right)}}{\prod_{k=1}^{n}\left(2-a_{(k) 1}^{l}\right)^{\mu\left(Z_{(k)}\right)-\mu\left(Z_{(k+1)}\right)}+\prod_{k=1}^{n}\left(a_{(k) 1}^{l}\right)^{\mu\left(Z_{(k)}\right)-\mu\left(Z_{(k+1)}\right)}}, \frac{2 \prod_{k=1}^{n}\left(a_{(k) 2}^{l}\right)^{\mu\left(Z_{(k)}\right)-\mu\left(Z_{(k+1)}\right)}}{\prod_{k=1}^{n}\left(2-a_{(k) 2}^{l}\right)^{\mu\left(Z_{(k)}\right)-\mu\left(Z_{(k+1)}\right)}+\prod_{k=1}^{n}\left(a_{(k) 2}^{l}\right)^{\mu\left(Z_{(k)}\right)-\mu\left(Z_{(k+1)}\right)}}, \\
& \frac{2 \prod_{k=1}^{n}\left(a_{(k) 3}^{l}\right)^{\mu\left(Z_{(k)}\right)-\mu\left(Z_{(k+1)}\right)}}{\prod_{k=1}^{n}\left(2-a_{(k) 3}^{l}\right)^{\mu\left(Z_{(k)}\right)-\mu\left(Z_{(k+1)}\right)}+\prod_{k=1}^{n}\left(a_{(k) 3}^{l}\right)^{\mu\left(Z_{(k)}\right)-\mu\left(Z_{(k+1)}\right)}}, \frac{2 \prod_{k=1}^{n}\left(a_{(k) 4}^{l}\right)^{\mu\left(Z_{(k)}\right)-\mu\left(Z_{(k+1)}\right)}}{\prod_{k=1}^{n}\left(2-a_{(k) 4}^{l}\right)^{\mu\left(Z_{(k)}\right)-\mu\left(Z_{(k+1)}\right)}+\prod_{k=1}^{n}\left(a_{(k) 4}^{l}\right)^{\mu\left(Z_{(k)}\right)-\mu\left(Z_{(k+1)}\right)}} ; \\
& \left.\frac{2 \prod_{k=1}^{n}\left(h_{1}\left(A_{(k)}^{l}\right)\right)^{\mu\left(Z_{(k)}\right)-\mu\left(Z_{(k+1)}\right)}}{\prod_{k=1}^{n}\left(2-h_{1}\left(A_{(k)}^{l}\right)\right)^{\mu\left(Z_{(k)}\right)-\mu\left(Z_{(k+1)}\right)}+\prod_{k=1}^{n}\left(h_{1}\left(A_{(k)}^{l}\right)\right)^{\mu\left(Z_{(k)}\right)-\mu\left(Z_{(k+1)}\right)}}, \frac{2 \prod_{k=1}^{n}\left(h_{2}\left(A_{(k)}^{l}\right)\right)^{\mu\left(Z_{(k)}\right)-\mu\left(Z_{(k+1)}\right)}}{\left.\prod_{k=1}^{n}\left(2-h_{2}\left(A_{(k)}^{l}\right)\right)^{\mu\left(Z_{(k)}\right)-\mu\left(Z_{(k+1)}\right)}+\prod_{k=1}^{n}\left(h_{2}\left(A_{(k)}^{l}\right)\right)^{\mu\left(Z_{(k)}\right)-\mu\left(Z_{(k+1)}\right)}\right)}\right)
\end{aligned}
$$

(3) When we utilize the Frank operations, the IT2FAC operator becomes the Frank IT2FC (FIT2FC) operator, i.e.: 


$$
\begin{aligned}
& F I T 2 F C\left(A_{1}, A_{2}, \ldots, A_{n}\right) \\
&=\left(\left(\log _{\gamma}\left(1+\prod_{k=1}^{n}\left(\gamma^{a_{(k) 1}^{u}}-1\right)^{\mu\left(Z_{(k)}\right)-\mu\left(Z_{(k+1)}\right)}\right), \log _{\gamma}\left(1+\prod_{k=1}^{n}\left(\gamma^{a_{(k) 2}^{u}}-1\right)^{\mu\left(Z_{(k)}\right)-\mu\left(Z_{(k+1)}\right)}\right),\right.\right. \\
& \log _{\gamma}\left(1+\prod_{k=1}^{n}\left(\gamma^{a_{(k) 3}^{u}}-1\right)^{\mu\left(Z_{(k)}\right)-\mu\left(Z_{(k+1)}\right)}\right), \log _{\gamma}\left(1+\prod_{k=1}^{n}\left(\gamma^{a_{(k) 4}^{u}}-1\right)^{\mu\left(Z_{(k)}\right)-\mu\left(Z_{(k+1)}\right)}\right) \\
&\left.\log _{\gamma}\left(1+\prod_{k=1}^{n}\left(\gamma^{h_{1}\left(A_{(k)}^{u}\right)}-1\right)^{\mu\left(Z_{(k)}\right)-\mu\left(Z_{(k+1)}\right)}\right), \log _{\gamma}\left(1+\prod_{k=1}^{n}\left(\gamma^{h_{2}\left(A_{(k)}^{u}\right)}-1\right)^{\mu\left(Z_{(k)}\right)-\mu\left(Z_{(k+1)}\right)}\right)\right), \\
&\left(\log _{\gamma}\left(1+\prod_{k=1}^{n}\left(\gamma^{a_{(k) 1}^{l}}-1\right)^{\mu\left(Z_{(k)}\right)-\mu\left(Z_{(k+1)}\right)}\right), \log _{\gamma}\left(1+\prod_{k=1}^{n}\left(\gamma^{a_{(k) 2}^{l}}-1\right)^{\mu\left(Z_{(k)}\right)-\mu\left(Z_{(k+1)}\right)}\right),\right. \\
& \log _{\gamma}\left(1+\prod_{k=1}^{n}\left(\gamma^{a_{(k) 3}^{l}}-1\right)^{\mu\left(Z_{(k)}\right)-\mu\left(Z_{(k+1)}\right)}\right), \log _{\gamma}\left(1+\prod_{k=1}^{n}\left(\gamma^{a_{(k) 4}^{l}}-1\right)^{\mu\left(Z_{(k)}\right)-\mu\left(Z_{(k+1)}\right)}\right) \\
&\left.\left.\log _{\gamma}\left(1+\prod_{k=1}^{n}\left(\gamma^{h_{1}\left(A_{(k)}^{l}\right)}-1\right)^{\mu\left(Z_{(k)}\right)-\mu\left(Z_{(k+1)}\right)}\right), \log _{\gamma}\left(1+\prod_{k=1}^{n}\left(\gamma^{h_{2}\left(A_{(k)}^{l}\right)}-1\right)^{\mu\left(Z_{(k)}\right)-\mu\left(Z_{(k+1)}\right)}\right)\right)\right)
\end{aligned}
$$

(4) When we utilize the Aczel-Alsina operations, the IT2FAC operator becomes the Aczel-Alsina IT2FC (AAIT2FC) operator, i.e.:

$$
\begin{aligned}
& \operatorname{AAIT} 2 F C\left(A_{1}, A_{2}, \ldots, A_{n}\right) \\
& =\left(\left(e^{-\left(\sum_{k=1}^{n}\left(\mu\left(Z_{(k)}\right)-\mu\left(Z_{(k+1)}\right)\right)\left(-\ln a_{(k) 1}^{u}\right)^{\gamma}\right)^{\frac{1}{\gamma}}}, e^{-\left(\sum_{k=1}^{n}\left(\mu\left(Z_{(k)}\right)-\mu\left(Z_{(k+1)}\right)\right)\left(-\ln a_{(k) 2}^{u}\right)^{\gamma}\right)^{\frac{1}{\gamma}}}, e^{-\left(\sum_{k=1}^{n}\left(\mu\left(Z_{(k)}\right)-\mu\left(Z_{(k+1)}\right)\right)\left(-\ln a_{(k) 3}^{u}\right)^{\gamma}\right)^{\frac{1}{\gamma}}},\right.\right. \\
& \left.e^{-\left(\sum_{k=1}^{n}\left(\mu\left(Z_{(k)}\right)-\mu\left(Z_{(k+1)}\right)\right)\left(-\ln a_{(k) 4}^{u}\right)^{\gamma}\right)^{\frac{1}{\gamma}}} ; e^{-\left(\sum_{k=1}^{n}\left(\mu\left(Z_{(k)}\right)-\mu\left(Z_{(k+1)}\right)\right)\left(-\ln h_{1}\left(A_{(k)}^{u}\right)\right)^{\gamma}\right)^{\frac{1}{\gamma}}}, e^{-\left(\sum_{k=1}^{n}\left(\mu\left(Z_{(k)}\right)-\mu\left(Z_{(k+1)}\right)\right)\left(-\ln h_{2}\left(A_{(k)}^{u}\right)\right)^{\gamma}\right)^{\frac{1}{\gamma}}}\right), \\
& \left(e^{-\left(\sum_{k=1}^{n}\left(\mu\left(Z_{(k)}\right)-\mu\left(Z_{(k+1)}\right)\right)\left(-\ln a_{(k) 1}^{l}\right)^{\gamma}\right)^{\frac{1}{\gamma}}}, e^{-\left(\sum_{k=1}^{n}\left(\mu\left(Z_{(k)}\right)-\mu\left(Z_{(k+1)}\right)\right)\left(-\ln a_{(k) 2}^{l}\right)^{\gamma}\right)^{\frac{1}{\gamma}}}, e^{-\left(\sum_{k=1}^{n}\left(\mu\left(Z_{(k)}\right)-\mu\left(Z_{(k+1)}\right)\right)\left(-\ln a_{(k) 3}^{l}\right)^{\gamma}\right)^{\frac{1}{\gamma}}},\right. \\
& \left.\left.e^{-\left(\sum_{k=1}^{n}\left(\mu\left(Z_{(k)}\right)-\mu\left(Z_{(k+1)}\right)\right)\left(-\ln a_{(k) 4}^{l}\right)^{\gamma}\right)^{\frac{1}{\gamma}}} ; e^{-\left(\sum_{k=1}^{n}\left(\mu\left(Z_{(k)}\right)-\mu\left(Z_{(k+1)}\right)\right)\left(-\ln h_{1}\left(A_{(k)}^{l}\right)\right)^{\gamma}\right)^{\frac{1}{\gamma}}}, e^{-\left(\sum_{k=1}^{n}\left(\mu\left(Z_{(k)}\right)-\mu\left(Z_{(k+1)}\right)\right)\left(-\ln h_{2}\left(A_{(k)}^{l}\right)\right)^{\gamma}\right)^{\frac{1}{\gamma}}}\right)\right) .
\end{aligned}
$$

To illustrate how to use the developed operators, the following case is given.

Example 1. It is assumed that an expert assesses the alternative $p_{1}$ concerning the criteria $z_{1}, z_{2}$ and $z_{3}$. The FMs of the criteria set $\left\{z_{1}, z_{2}, z_{3}\right\}$ are: $\mu(\phi)=0, \quad \mu\left(\left\{z_{1}\right\}\right)=0.4, \quad \mu\left(\left\{z_{2}\right\}\right)=0.2, \quad \mu\left(\left\{z_{3}\right\}\right)=0.3$, $\mu\left(\left\{z_{1}, z_{2}\right\}\right)=0.5, \quad \mu\left(\left\{z_{1}, z_{3}\right\}\right)=0.7, \quad \mu\left(\left\{z_{2}, z_{3}\right\}\right)=0.6$ and $\mu\left(\left\{z_{1}, z_{2}, z_{3}\right\}\right)=1$. The criteria values are: $A_{1}=((0,0,0,0.1 ; 1,1),(0,0,0,0.05 ; 0.9,0.9)), \quad A_{2}=((0.15,0.3,0.35,0.5 ; 1,1),(0.2,0.25,0.3,0.4 ; 0.9,0.9))$, and $A_{3}=((0,0.1,0.15,0.3 ; 1,1),(0.05,0.1,0.1,0.2 ; 0.9,0.9))$. If The IT2FC operator is applied, we can get a collective value $A=\left(\left(a_{1}^{u}, a_{2}^{u}, a_{3}^{u}, a_{4}^{u} ; h_{1}\left(A^{u}\right), h_{2}\left(A^{u}\right)\right),\left(a_{1}^{l}, a_{2}^{l}, a_{3}^{l}, a_{4}^{l} ; h_{1}\left(A^{l}\right), h_{2}\left(A^{l}\right)\right)\right)$. The detailed steps are depicted as follows:

By Definition 4, we get $A_{1}<A_{3}<A_{2}$. Then, we get $\mu\left(Z_{(1)}\right)-\mu\left(Z_{(2)}\right)=\mu\left(\left\{z_{1}, z_{2}, z_{3}\right\}\right)-\mu\left(\left\{z_{2}, z_{3}\right\}\right)=0.4$, $\mu\left(Z_{(2)}\right)-\mu\left(Z_{(3)}\right)=\mu\left(\left\{z_{2}, z_{3}\right\}\right)-\mu\left(\left\{z_{2}\right\}\right)=0.4$ and $\mu\left(Z_{(3)}\right)-\mu(\phi)=\mu\left(\left\{z_{2}\right\}\right)-\mu(\phi)=0.2$. Besides, by 
the operations of IT2FNs, we get $a_{1}^{u}=\left(a_{11}^{u}\right)^{\mu\left(Z_{(1)}\right)-\mu\left(Z_{(2)}\right)} \times\left(a_{13}^{u}\right)^{\mu\left(Z_{(2)}\right)-\mu\left(Z_{(3)}\right)} \times\left(a_{12}^{u}\right)^{\mu\left(Z_{(3)}\right)-\mu(\phi)}=0$.

Similarly, it is obtained: $\quad a_{2}^{u}=0, \quad a_{3}^{u}=0, \quad a_{4}^{u}=0.2141, \quad h_{1}\left(A^{u}\right)=1, \quad h_{2}\left(A^{u}\right)=1, \quad a_{1}^{l}=0$, $a_{2}^{l}=0, \quad a_{3}^{l}=0, \quad a_{4}^{l}=0.132, \quad h_{1}\left(A^{l}\right)=0.9$ and $h_{2}\left(A^{l}\right)=0.9$.

Therefore, we have:

$$
\begin{aligned}
& A=I T 2 F C\left(A_{1}, A_{2}, A_{3}\right) \\
& =\left(\left(\prod_{k=1}^{3}\left(a_{(k) 1}^{u}\right)^{\mu\left(\mathbf{Z}_{(k)}\right)-\mu\left(\mathbf{Z}_{(k+1)}\right)}, \prod_{k=1}^{3}\left(a_{(k) 2}^{u}\right)^{\mu\left(\mathbf{Z}_{(k)}\right)-\mu\left(\mathbf{Z}_{(k+1)}\right)}, \prod_{k=1}^{3}\left(a_{(k) 3}^{u}\right)^{\mu\left(\mathbf{Z}_{(k)}\right)-\mu\left(\mathbf{Z}_{(k+1)}\right)}, \prod_{k=1}^{3}\left(a_{(k) 4}^{u}\right)^{\mu\left(\mathbf{Z}_{(k)}\right)-\mu\left(\mathbf{Z}_{(k+1)}\right)} ;\right.\right. \\
& \left.\prod_{k=1}^{3}\left(h_{1}\left(A_{(k)}^{u}\right)\right)^{\mu\left(Z_{(k)}\right)-\mu\left(Z_{(k+1)}\right)}, \prod_{k=1}^{3}\left(h_{2}\left(A_{(k)}^{u}\right)\right)^{\mu\left(Z_{(k)}\right)-\mu\left(Z_{(k+1)}\right)}\right), \\
& \left(\prod_{k=1}^{3}\left(a_{(k) 1}^{l}\right)^{\mu\left(Z_{(k)}\right)-\mu\left(Z_{(k+1)}\right)}, \prod_{k=1}^{3}\left(a_{(k) 2}^{l}\right)^{\mu\left(Z_{(k)}\right)-\mu\left(Z_{(k+1)}\right)}, \prod_{k=1}^{3}\left(a_{(k) 3}^{l}\right)^{\mu\left(Z_{(k k)}\right)-\mu\left(Z_{(k+1)}\right)}, \prod_{k=1}^{3}\left(a_{(k) 4}^{l}\right)^{\mu\left(Z_{(k)}\right)-\mu\left(Z_{(k+1)}\right)} ;\right. \\
& \left.\left.\prod_{k=1}^{3}\left(h_{1}\left(A_{(k)}^{l}\right)\right)^{\mu\left(Z_{(k)}\right)-\mu\left(Z_{(k+1)}\right)}, \prod_{k=1}^{3}\left(h_{2}\left(A_{(k)}^{l}\right)\right)^{\mu\left(Z_{(k)}\right)-\mu\left(Z_{(k+1)}\right)}\right)\right) \\
& =((0,0,0,0.2141 ; 1,1),(0,0,0,0.132 ; 0.9,0.9)) \text {. }
\end{aligned}
$$

\subsection{The BIT2FAC operator}

Note that the proposed IT2FAC operator only captures the interaction between adjacent coalitions of attributes, $Z_{(k)}$ and $Z_{(k+1)}(k=1,2, \ldots, n)$. For instance, suppose that $Z=\left\{z_{1}, z_{2}, z_{3}\right\}$ and $A_{1}<A_{2}<A_{3}$; then, it is:

$$
\begin{aligned}
& \operatorname{IT} 2 F A C\left(A_{1}, A_{2}, A_{3}\right) \\
& =\left(\left(g^{-1}\left(\left(\mu\left(\left\{z_{1}, z_{2}, z_{3}\right\}\right)-\mu\left(\left\{z_{2}, z_{3}\right\}\right)\right) g\left(a_{11}^{u}\right)+\left(\mu\left(\left\{z_{2}, z_{3}\right\}\right)-\mu\left(\left\{z_{3}\right\}\right)\right) g\left(a_{21}^{u}\right)+\mu\left(\left\{z_{3}\right\}\right) g\left(a_{31}^{u}\right)\right)\right.\right. \text {, } \\
& g^{-1}\left(\left(\mu\left(\left\{z_{1}, z_{2}, z_{3}\right\}\right)-\mu\left(\left\{z_{2}, z_{3}\right\}\right)\right) g\left(a_{12}^{u}\right)+\left(\mu\left(\left\{z_{2}, z_{3}\right\}\right)-\mu\left(\left\{z_{3}\right\}\right)\right) g\left(a_{22}^{u}\right)+\mu\left(\left\{z_{3}\right\}\right) g\left(a_{32}^{u}\right)\right), \\
& g^{-1}\left(\left(\mu\left(\left\{z_{1}, z_{2}, z_{3}\right\}\right)-\mu\left(\left\{z_{2}, z_{3}\right\}\right)\right) g\left(a_{13}^{u}\right)+\left(\mu\left(\left\{z_{2}, z_{3}\right\}\right)-\mu\left(\left\{z_{3}\right\}\right)\right) g\left(a_{23}^{u}\right)+\mu\left(\left\{z_{3}\right\}\right) g\left(a_{33}^{u}\right)\right), \\
& g^{-1}\left(\left(\mu\left(\left\{z_{1}, z_{2}, z_{3}\right\}\right)-\mu\left(\left\{z_{2}, z_{3}\right\}\right)\right) g\left(a_{14}^{u}\right)+\left(\mu\left(\left\{z_{2}, z_{3}\right\}\right)-\mu\left(\left\{z_{3}\right\}\right)\right) g\left(a_{24}^{u}\right)+\mu\left(\left\{z_{3}\right\}\right) g\left(a_{34}^{u}\right)\right) ; \\
& g^{-1}\left(\left(\mu\left(\left\{z_{1}, z_{2}, z_{3}\right\}\right)-\mu\left(\left\{z_{2}, z_{3}\right\}\right)\right) g\left(h_{1}\left(A_{1}^{u}\right)\right)+\left(\mu\left(\left\{z_{2}, z_{3}\right\}\right)-\mu\left(\left\{z_{3}\right\}\right)\right) g\left(h_{1}\left(A_{2}^{u}\right)\right)+\mu\left(\left\{z_{3}\right\}\right) g\left(h_{1}\left(A_{3}^{u}\right)\right)\right), \\
& \left.g^{-1}\left(\left(\mu\left(\left\{z_{1}, z_{2}, z_{3}\right\}\right)-\mu\left(\left\{z_{2}, z_{3}\right\}\right)\right) g\left(h_{2}\left(A_{1}^{u}\right)\right)+\left(\mu\left(\left\{z_{2}, z_{3}\right\}\right)-\mu\left(\left\{z_{3}\right\}\right)\right) g\left(h_{2}\left(A_{2}^{u}\right)\right)+\mu\left(\left\{z_{3}\right\}\right) g\left(h_{2}\left(A_{3}^{u}\right)\right)\right)\right), \\
& \left(g^{-1}\left(\left(\mu\left(\left\{z_{1}, z_{2}, z_{3}\right\}\right)-\mu\left(\left\{z_{2}, z_{3}\right\}\right)\right) g\left(a_{11}^{l}\right)+\left(\mu\left(\left\{z_{2}, z_{3}\right\}\right)-\mu\left(\left\{z_{3}\right\}\right)\right) g\left(a_{21}^{l}\right)+\mu\left(\left\{z_{3}\right\}\right) g\left(a_{31}^{l}\right)\right),\right. \\
& g^{-1}\left(\left(\mu\left(\left\{z_{1}, z_{2}, z_{3}\right\}\right)-\mu\left(\left\{z_{2}, z_{3}\right\}\right)\right) g\left(a_{12}^{l}\right)+\left(\mu\left(\left\{z_{2}, z_{3}\right\}\right)-\mu\left(\left\{z_{3}\right\}\right)\right) g\left(a_{22}^{l}\right)+\mu\left(\left\{z_{3}\right\}\right) g\left(a_{32}^{l}\right)\right), \\
& g^{-1}\left(\left(\mu\left(\left\{z_{1}, z_{2}, z_{3}\right\}\right)-\mu\left(\left\{z_{2}, z_{3}\right\}\right)\right) g\left(a_{13}^{l}\right)+\left(\mu\left(\left\{z_{2}, z_{3}\right\}\right)-\mu\left(\left\{z_{3}\right\}\right)\right) g\left(a_{23}^{l}\right)+\mu\left(\left\{z_{3}\right\}\right) g\left(a_{33}^{l}\right)\right), \\
& g^{-1}\left(\left(\mu\left(\left\{z_{1}, z_{2}, z_{3}\right\}\right)-\mu\left(\left\{z_{2}, z_{3}\right\}\right)\right) g\left(a_{14}^{l}\right)+\left(\mu\left(\left\{z_{2}, z_{3}\right\}\right)-\mu\left(\left\{z_{3}\right\}\right)\right) g\left(a_{24}^{l}\right)+\mu\left(\left\{z_{3}\right\}\right) g\left(a_{34}^{l}\right)\right) ; \\
& g^{-1}\left(\left(\mu\left(\left\{z_{1}, z_{2}, z_{3}\right\}\right)-\mu\left(\left\{z_{2}, z_{3}\right\}\right)\right) g\left(h_{1}\left(A_{1}^{l}\right)\right)+\left(\mu\left(\left\{z_{2}, z_{3}\right)-\mu\left(\left\{z_{3}\right\}\right)\right) g\left(h_{1}\left(A_{2}^{l}\right)\right)+\mu\left(\left\{z_{3}\right\}\right) g\left(h_{1}\left(A_{3}^{l}\right)\right)\right),\right. \\
& \left.g^{-1}\left(\left(\mu\left(\left\{z_{1}, z_{2}, z_{3}\right\}\right)-\mu\left(\left\{z_{2}, z_{3}\right\}\right)\right) g\left(h_{2}\left(A_{1}^{l}\right)\right)+\left(\mu\left(\left\{z_{2}, z_{3}\right)-\mu\left(\left\{z_{3}\right\}\right)\right) g\left(h_{2}\left(A_{2}^{l}\right)\right)+\mu\left(\left\{z_{3}\right\}\right) g\left(h_{2}\left(A_{3}^{l}\right)\right)\right)\right)\right) \text {. }
\end{aligned}
$$

Obviously, the proposed IT2FAC operator only captures the coalitions of attributes $Z_{(1)}=\left\{z_{1}, z_{2}, z_{3}\right\}$, $Z_{(2)}=\left\{z_{2}, z_{3}\right\}$ and $Z_{(3)}=\left\{z_{3}\right\}$. Actually, we also need to consider the coalitions of attributes $\left\{z_{1}\right\}$, $\left\{z_{2}\right\},\left\{z_{1}, z_{2}\right\}$ and $\left\{z_{1}, z_{3}\right\}$. Thus, the proposed IT2FAC operator may not always be appropriate 
because some coalitions of attributes of interest might not be considered. In what follows, to globally capture the various coalitions of attributes, we extend the generalized Banzhaf index to IT2FAC operator, and then propose the BIT2FAC operator.

The generalized Banzhaf index below is an interaction index presented by Marichal [27]:

$$
\psi(B)=\sum_{D \subset Z \backslash B} \frac{1}{2^{n-|B|}}(\mu(B \cup D)-\mu(D)),
$$

where $\mu$ denotes an FM on the set $Z, B$ denotes any subset of $Z, \quad Z \backslash B$ denotes the difference set between $Z$ and $B,|B|$ and $n$ are the cardinalities of the set $B$ and $Z$, respectively, and $D$ denotes any subset of $Z \backslash B$. Through Eq. (22), we find that when there is only one element $z_{i}$ in the set $B$, i.e., $B=\left\{z_{i}\right\}$, Eq. (22) becomes the Banzhaf function [27]:

$$
\psi\left(\left\{z_{i}\right\}\right)=\sum_{\left.D \subset Z \backslash z_{i}\right\}} \frac{1}{2^{n-1}}\left(\mu\left(\left\{z_{i}\right\} \cup D\right)-\mu(D)\right) .
$$

Eq. (22) represents an expected value of the overall interaction between the coalition $B$ and any coalitions in $Z \backslash B$, which in a MADM would be considered as the weight (importance) of the attribute set $B$. Additionally, Meng et al. [31] pointed out that the generalized Banzhaf index is also a special type of FM. Thus, we extend the Choquet integral with generalized Banzhaf index to handle IT2FNs and propose the BIT2FAC operator to globally capture the interactions of the attributes.

Definition 8. It is assumed that $Z=\left\{z_{1}, z_{2}, \ldots, z_{n}\right\}$ is a collection of attributes and $\Lambda=\left\{A_{1}, A_{2}, \ldots, A_{n}\right\}$ is a set of trapezoidal IT2FNs on $Z$. The BIT2FAC operator is:

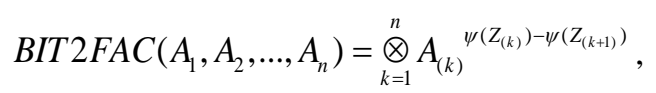

where $A_{(k)}=\left(A_{(k)}^{u}, A_{(k)}^{l}\right)$ denotes the $k$-th smallest element in the set $\Lambda=\left\{A_{1}, A_{2}, \ldots, A_{n}\right\}, z_{(k)}$ is the attribute corresponding to $A_{(k)}, Z_{(k)}=\left\{z_{(k)}, z_{(k+1)}, \ldots, z_{(n)}\right\}$ with $Z_{(n+1)}=\phi$, and $\psi\left(Z_{(k)}\right)(k=1,2, \ldots, n+1)$ represents the generalized Banzhaf index on the criteria set $Z_{(k)}$.

Based on the operations of IT2FNs listed in Eqs. (6)-(7), from Eq. (24), we derive the following result.

Theorem 3. The aggregation output of the set $\Lambda=\left\{A_{1}, A_{2}, \ldots, A_{n}\right\}$ of trapezoidal IT2FNs by the BIT2FAC operator is the following trapezoidal IT2FN: 


$$
\begin{aligned}
& \operatorname{BIT} 2 \text { FAC }\left(A_{1}, A_{2}, \ldots, A_{n}\right) \\
&=\left(\left(g^{-1}\left(\sum_{k=1}^{n}\left(\psi\left(Z_{(k)}\right)-\psi\left(Z_{(k+1)}\right)\right) g\left(a_{(k) 1}^{u}\right)\right), g^{-1}\left(\sum_{k=1}^{n}\left(\psi\left(Z_{(k)}\right)-\psi\left(Z_{(k+1)}\right)\right) g\left(a_{(k) 2}^{u}\right)\right),\right.\right. g^{-1}\left(\sum_{k=1}^{n}\left(\psi\left(Z_{(k)}\right)-\psi\left(Z_{(k+1)}\right)\right) g\left(a_{(k) 3}^{u}\right)\right), g^{-1}\left(\sum_{k=1}^{n}\left(\psi\left(Z_{(k)}\right)-\psi\left(Z_{(k+1)}\right)\right) g\left(a_{(k) 4}^{u}\right)\right) ; \\
&\left.g^{-1}\left(\sum_{k=1}^{n}\left(\psi\left(Z_{(k)}\right)-\psi\left(Z_{(k+1)}\right)\right) g\left(h_{1}\left(A_{(k)}^{u}\right)\right)\right), g^{-1}\left(\sum_{k=1}^{n}\left(\psi\left(Z_{(k)}\right)-\psi\left(Z_{(k+1)}\right)\right) g\left(h_{2}\left(A_{(k)}^{u}\right)\right)\right)\right) \\
& \quad\left(g^{-1}\left(\sum_{k=1}^{n}\left(\psi\left(Z_{(k)}\right)-\psi\left(Z_{(k+1)}\right)\right) g\left(a_{(k) 1}^{l}\right)\right), g^{-1}\left(\sum_{k=1}^{n}\left(\psi\left(Z_{(k)}\right)-\psi\left(Z_{(k+1)}\right)\right) g\left(a_{(k) 2}^{l}\right)\right),\right. \\
& g^{-1}\left(\sum_{k=1}^{n}\left(\psi\left(Z_{(k)}\right)-\psi\left(Z_{(k+1)}\right)\right) g\left(a_{(k) 3}^{l}\right)\right), g^{-1}\left(\sum_{k=1}^{n}\left(\psi\left(Z_{(k)}\right)-\psi\left(Z_{(k+1)}\right)\right) g\left(a_{(k) 4}^{l}\right)\right) ; \\
&\left.\left.g^{-1}\left(\sum_{k=1}^{n}\left(\psi\left(Z_{(k)}\right)-\psi\left(Z_{(k+1)}\right)\right) g\left(h_{1}\left(A_{(k)}^{l}\right)\right)\right), g^{-1}\left(\sum_{k=1}^{n}\left(\psi\left(Z_{(k)}\right)-\psi\left(Z_{(k+1)}\right)\right) g\left(h_{2}\left(A_{(k)}^{l}\right)\right)\right)\right)\right) .
\end{aligned}
$$

The proof of Theorem 3 is similar to the proof of Theorem 1; thus, it is omitted here.

Corollary 3. When all elements $z_{k}(k=1,2, \ldots, n)$ in the set $Z$ are independent, the generalized Banzhaf index $\psi(D)$ is an additive measure, i.e., $\psi(D)=\sum_{z_{k} \in D} \mu\left(\left\{z_{k}\right\}\right)$ for any $D \subset Z$, and the BIT2FAC operator becomes the IT2FAWA operator.

Corollary 4. If $\psi(D)=\sum_{k=1}^{|D|} w_{k}$ for any $D \subset Z$, where $|D|$ represents the cardinality of $D$, then the BIT2FAC operator becomes the IT2FAOWA operator.

Similar properties to those proved for the IT2FAC operator, can also be proved for the BIT2FAC operator. Their proofs are therefore omitted here. In addition, for the additive generators of Table 1, the following BIT2FAC operators are derived: the Banzhaf IT2FC operator (algebraic); the Banzhaf EIT2FC operator (Einstein); the Banzhaf FIT2FC (Frank); and the Banzhaf AAIT2FC operator (Aczel-Alsina). The expressions of these four operators can be derived by replacing $\mu\left(Z_{(k)}\right)-\mu\left(Z_{(k+1)}\right)$ in Eqs. (18)-(21) with $\psi\left(Z_{(k)}\right)-\psi\left(Z_{(k+1)}\right)$, and therefore they are omitted here.

\subsection{The 2ABIT2FAC operator}

FMs have an effect on the computational complexity of problems where it becomes exponential because of the involvement of the cardinality of the power set of a set. As a result, when the cardinality of the set $Z$ is high, it is not easy to derive the FMs of all its coalitions. The proposed BIT2FAC operator is based on FMs, and therefore its computational complexity is also exponential. To improve the practicability of the proposed BIT2FAC operator, we shall propose the 2ABIT2FAC operator using 2-additive fuzzy measures (2AFMs) [16].

For 2AFM, given any $S \subset Z$ with $|S| \geq 2$ :

$$
\begin{aligned}
u(S) & =\sum_{j=1}^{n} e_{\left\{z_{j}\right\}} \zeta_{j}+\sum_{\left\{z_{j}, z_{i}\right\} \subset Z} e_{\left\{z_{j}, z_{i}\right\}} \zeta_{j} \zeta_{i}=\sum_{z_{j} \in S} e_{\left\{z_{j}\right\}}+\sum_{\left\{z_{j}, z_{i}\right\} \subset S} e_{\left\{z_{j}, z_{i}\right\}} \\
& =\sum_{\left\{z_{j}, z_{i}\right\} \subset S} \mu\left(\left\{z_{j}, z_{i}\right\}\right)-(|S|-2) \sum_{z_{i} \in S} \mu\left(\left\{z_{i}\right\}\right),
\end{aligned}
$$

where $e_{\left\{z_{j}\right\}}$ and $e_{\left\{z_{j}, z_{i}\right\}}$ indicate the Möbius transform coefficients [16], $\mu\left(\left\{z_{j}\right\}\right)=e_{\left\{z_{j}\right\}}$, 
$\mu\left(\left\{z_{j}, z_{i}\right\}\right)=e_{\left(\left\{z_{j}\right\}\right)}+e_{\left(\left\{z_{i}\right\}\right)}+e_{\left(\left\{z_{j}, z_{i}\right\}\right)}, \zeta_{j}$ represents a Boolean variable $\left(\zeta_{j}=1 \Leftrightarrow \zeta_{j} \in S\right)$, and $|S|$ is the cardinality of the set $S$. Obviously, to derive 2AFM, only $n(n+1) / 2$ coefficients $\mu\left(\left\{z_{j}\right\}\right)$ and $\mu\left(\left\{z_{j}, z_{i}\right\}\right)$ are needed.

The following theorem was proved by Grabisch [16] to derive a 2AFM.

Theorem 4 [16]. Given the set $Z, \mu$ is a 2 AFM on $Z$ if for all $z_{j}, z_{i} \in Z$, the following conditions are verified:

(1) $\mu\left(\left\{z_{j}\right\}\right) \geq 0, \forall z_{j} \in Z$;

(2) $\sum_{\left\{z_{j}, z_{i}\right\} \subset Z} \mu\left(\left\{z_{j}, z_{i}\right\}\right)-(n-2) \sum_{\left\{z_{j}\right\} \subset Z} \mu\left(\left\{z_{j}\right\}\right)=1$;

(3) $\sum_{\left\{z_{j}\right\} \subset S \backslash z_{i}}\left(\mu\left(\left\{z_{j}, z_{i}\right\}\right)-\mu\left(\left\{z_{j}\right\}\right)\right) \geq(|S|-2) \mu\left(\left\{z_{i}\right\}\right), \quad \forall S \subset Z$ with $z_{i} \in S$ and $|S| \geq 2$.

Menget al. [31] presented the explicit expression of the generalized Banzhaf index with 2AFM below:

Theorem 5 [31]. It is assumed that $\mu$ denotes a 2AFM on the set $Z$. The generalized Banzhaf index with 2- additive measure $\mu$ is:

$$
\psi(B)=\sum_{\left\{z_{j}, z_{i}\right\} \subset B} \mu\left(\left\{z_{j}, z_{i}\right\}\right)+\sum_{z_{j} \in B, z_{i} \in Z \backslash B} \frac{1}{2}\left(\mu\left(\left\{z_{j}, z_{i}\right\}\right)-|B| \mu\left(\left\{z_{i}\right\}\right)\right)-\frac{n+|B|-4}{2} \sum_{z_{j} \in B} \mu\left(\left\{z_{j}\right\}\right),
$$

where $B$ denotes any subset of $Z, \quad Z \backslash B$ denotes the difference set between $Z$ and $B$, and $|B|$ is the cardinality of the coalitions $B$. When there is only one element $z_{i}$ in the set $B$, i.e., $B=\left\{z_{i}\right\}$, then, Eq. (27) becomes the Banzhaf function with 2AFM:

$$
\psi\left(\left\{z_{j}\right\}\right)=\frac{3-n}{2} \mu\left(\left\{z_{j}\right\}\right)+\sum_{z_{i} \in Z \backslash z_{j}} \frac{1}{2}\left(\mu\left(\left\{z_{j}, z_{i}\right\}\right)-\mu\left(\left\{z_{i}\right\}\right)\right) .
$$

Theorem 6 [31]. It is assumed that $\mu$ denotes a 2AFM on the set $Z$, and $\psi$ is the generalized Banzhaf index with $2 \mathrm{AFM} \mu$. Then,

$$
\psi\left(S \cup\left\{z_{j}\right\}\right)-\psi\left(\left\{z_{j}\right\}\right)=\psi(S),
$$

for any $z_{j} \in Z$ and any $S \subset Z$ with $z_{j} \notin S$.

By Theorem 6, the 2ABIT2FAC operator can be defined as below:

Definition 9. It is assumed that $Z=\left\{z_{1}, z_{2}, \ldots, z_{n}\right\}$ is a collection of attributes and $\Lambda=\left\{A_{1}, A_{2}, \ldots, A_{n}\right\}$ is a set of trapezoidal IT2FNs on $Z$. The 2ABIT2FAC operator expression is:

$$
2 A B I T 2 F A C\left(A_{1}, A_{2}, \ldots, A_{n}\right)=\bigotimes_{k=1}^{n} A_{k}^{\psi\left(\left\{z_{k}\right\}\right)},
$$

where $z_{k}$ is the attribute corresponding to $A_{k}$, and $\psi\left(\left\{z_{k}\right\}\right)(k=1,2, \ldots, n)$ represents the Banzhaf function with 2AFM on the criterion $z_{k}$.

Based on the operations of IT2FNs listed in Eqs. (6)-(7), from Eq. (30), we derive the following result.

Theorem 7. The aggregation output of the set $\Lambda=\left\{A_{1}, A_{2}, \ldots, A_{n}\right\}$ of trapezoidal IT2FNs by the 2ABIT2FAC operator is the trapezoidal IT2FN: 


$$
\begin{aligned}
& 2 \text { ABIT } 2 \text { FAC }\left(A_{1}, A_{2}, \ldots, A_{n}\right) \\
&=\left(\left(g^{-1}\left(\sum_{k=1}^{n} \psi\left(\left\{z_{k}\right\}\right) g\left(a_{k 1}^{u}\right)\right), g^{-1}\left(\sum_{k=1}^{n} \psi\left(\left\{z_{k}\right\}\right) g\left(a_{k 2}^{u}\right)\right), g^{-1}\left(\sum_{k=1}^{n} \psi\left(\left\{z_{k}\right\}\right) g\left(a_{k 3}^{u}\right)\right),\right.\right. \\
&\left.g^{-1}\left(\sum_{k=1}^{n} \psi\left(\left\{z_{k}\right\}\right) g\left(a_{k 4}^{u}\right)\right) ; g^{-1}\left(\sum_{k=1}^{n} \psi\left(\left\{z_{k}\right\}\right) g\left(h_{1}\left(A_{k}^{u}\right)\right)\right), g^{-1}\left(\sum_{k=1}^{n} \psi\left(\left\{z_{k}\right\}\right) g\left(h_{2}\left(A_{k}^{u}\right)\right)\right)\right), \\
& \quad\left(g^{-1}\left(\sum_{k=1}^{n} \psi\left(\left\{z_{k}\right\}\right) g\left(a_{k 1}^{l}\right)\right), g^{-1}\left(\sum_{k=1}^{n} \psi\left(\left\{z_{k}\right\}\right) g\left(a_{k 2}^{l}\right)\right), g^{-1}\left(\sum_{k=1}^{n} \psi\left(\left\{z_{k}\right\}\right)\left(a_{k 3}^{l}\right)\right),\right. \\
&\left.\left.g^{-1}\left(\sum_{k=1}^{n} \psi\left(\left\{z_{k}\right\}\right) g\left(a_{k 4}^{l}\right)\right) ; g^{-1}\left(\sum_{k=1}^{n} \psi\left(\left\{z_{k}\right\}\right) g\left(h_{1}\left(A_{k}^{l}\right)\right)\right), g^{-1}\left(\sum_{k=1}^{n} \psi\left(\left\{z_{k}\right\}\right) g\left(h_{2}\left(A_{k}^{l}\right)\right)\right)\right)\right) .
\end{aligned}
$$

The proof of Theorem 7 is similar to the proof of Theorem 1; thus, it is omitted here.

Similar properties to those proved for the IT2FAC operator, can also be proved for the 2ABIT2FAC operator and therefore their proofs are also omitted here. In addition, for the additive generators of Table 1, the following BIT2FAC operators are derived: the 2-additive Banzhaf IT2FC operator (algebraic); the 2-additive Banzhaf EIT2FC operator (Einstein); the 2-additive Banzhaf FIT2FC operator (Frank); and the 2-additive Banzhaf AAIT2FC operator (Aczel-Alsina). The expressions of these four operators can be derived by replacing $\mu\left(Z_{(k)}\right)-\mu\left(Z_{(k+1)}\right)$ in Eqs. (18)-(21) with $\psi\left(\left\{z_{(k)}\right\}\right)$, and therefore they are omitted here.

\section{Bi-directional projection-based models for the optimal fuzzy measures}

The projection method is a powerful tool for obtaining attributes' weights [19]. In what follows, we shall utilize the bi-directional projection measure within the context of IT2FNs for determining the optimal FMs on the attributes set.

\subsection{A novel bi-directional projection measure of IT2FNs}

To develop the bi-directional projection measure of IT2FNs, based on the cosine measure and projection measure of picture fuzzy sets defined by Wang et al. [44], we first develop the cosine measure and general projection measure of IT2FNs.

Definition 10. It is assumed that $A=\left(A^{u}, A^{l}\right)$ is a trapezoidal IT2FN. The module of $A$ is:

$$
|A|=\sqrt{\left(\sum_{k=1}^{4}\left(a_{k}^{u}\right)^{2}+\sum_{k=1}^{4}\left(a_{k}^{l}\right)^{2}\right) \cdot\left(\sum_{k=1}^{2}\left(h_{k}\left(A^{u}\right)\right)^{2}+\sum_{k=1}^{2}\left(h_{k}\left(A^{l}\right)\right)^{2}\right)} .
$$

Definition 11. It is assumed that $A_{1}=\left(A_{1}^{u}, A_{1}^{l}\right)$ and $A_{2}=\left(A_{2}^{u}, A_{2}^{l}\right)$ are two trapezoidal IT2FNs. The inner product of $A_{1}$ and $A_{2}$ is:

$$
A_{1} \cdot A_{2}=\left(\sum_{k=1}^{4} a_{1 k}^{u} a_{2 k}^{u}+\sum_{k=1}^{4} a_{1 k}^{l} a_{2 k}^{l}\right) \cdot\left(\sum_{k=1}^{2} h_{k}\left(A_{1}^{u}\right) h_{k}\left(A_{2}^{u}\right)+\sum_{k=1}^{2} h_{k}\left(A_{1}^{l}\right) h_{k}\left(A_{2}^{l}\right)\right) .
$$

Definition 12. It is assumed that $A_{1}=\left(A_{1}^{u}, A_{1}^{l}\right)$ and $A_{2}=\left(A_{2}^{u}, A_{2}^{l}\right)$ are two trapezoidal IT2FNs. The cosine of the angle between $A_{1}$ and $A_{2}$ is: 


$$
\operatorname{Cos}\left(A_{1}, A_{2}\right)=\frac{A_{1} \cdot A_{2}}{\left|A_{1}\right|\left|A_{2}\right|}=\frac{\left(\sum_{k=1}^{4} a_{1 k}^{u} a_{2 k}^{u}+\sum_{k=1}^{4} a_{1 k}^{l} a_{2 k}^{l}\right) \cdot\left(\sum_{k=1}^{2} h_{k}\left(A_{1}^{u}\right) h_{k}\left(A_{2}^{u}\right)+\sum_{k=1}^{2} h_{k}\left(A_{1}^{l}\right) h_{k}\left(A_{2}^{l}\right)\right)}{\prod_{t=1}^{2} \sqrt{\left(\sum_{k=1}^{4}\left(a_{t k}^{u}\right)^{2}+\sum_{k=1}^{4}\left(a_{t k}^{l}\right)^{2}\right) \cdot\left(\sum_{k=1}^{2}\left(h_{k}\left(A_{t}^{u}\right)\right)^{2}+\sum_{k=1}^{2}\left(h_{k}\left(A_{t}^{l}\right)\right)^{2}\right)}} .
$$

Definition 13. It is assumed that $A_{1}=\left(A_{1}^{u}, A_{1}^{l}\right)$ and $A_{2}=\left(A_{2}^{u}, A_{2}^{l}\right)$ are two trapezoidal IT2FNs. The projection of $A_{1}$ on $A_{2}$ is:

$$
\operatorname{Poj}_{A_{2}}\left(A_{1}\right)=\left|A_{1}\right| \operatorname{Cos}\left(A_{1}, A_{2}\right)=\frac{\left(\sum_{k=1}^{4} a_{1 k}^{u} a_{2 k}^{u}+\sum_{k=1}^{4} a_{1 k}^{l} a_{2 k}^{l}\right) \cdot\left(\sum_{k=1}^{2} h_{k}\left(A_{1}^{u}\right) h_{k}\left(A_{2}^{u}\right)+\sum_{k=1}^{2} h_{k}\left(A_{1}^{l}\right) h_{k}\left(A_{2}^{l}\right)\right)}{\sqrt{\left(\sum_{k=1}^{4}\left(a_{2 k}^{u}\right)^{2}+\sum_{k=1}^{4}\left(a_{2 k}^{l}\right)^{2}\right) \cdot\left(\sum_{k=1}^{2}\left(h_{k}\left(A_{2}^{u}\right)\right)^{2}+\sum_{k=1}^{2}\left(h_{k}\left(A_{2}^{l}\right)\right)^{2}\right)}} .
$$

The projection measure $\operatorname{Poj}_{A_{2}}\left(A_{1}\right)$ reflects both the distance and the angle between $A_{1}$ and $A_{2}$. Generally, the larger the value of $\operatorname{Poj}_{A_{2}}\left(A_{1}\right)$ is, the closer $A_{1}$ and $A_{2}$ are.

On the basis of the developed projection measure between IT2FNs, a bi-directional projection measure of IT2FNs is defined below:

Definition 14. It is assumed that $A_{1}=\left(A_{1}^{u}, A_{1}^{l}\right)$ and $A_{2}=\left(A_{2}^{u}, A_{2}^{l}\right)$ are two trapezoidal IT2FNs. The bi-directional projection between $A_{1}$ and $A_{2}$ is:

$\operatorname{Bpoj}\left(A_{1}, A_{2}\right)=\frac{2 A_{1} \cdot A_{2}}{\left|A_{1}\right|^{2}+\left|A_{2}\right|^{2}}=\frac{2 \times\left(\sum_{k=1}^{4} a_{1 k}^{u} a_{2 k}^{u}+\sum_{k=1}^{4} a_{1 k}^{l} a_{2 k}^{l}\right) \cdot\left(\sum_{k=1}^{2} h_{k}\left(A_{1}^{u}\right) h_{k}\left(A_{2}^{u}\right)+\sum_{k=1}^{2} h_{k}\left(A_{1}^{l}\right) h_{k}\left(A_{2}^{l}\right)\right)}{\sum_{t=1}^{2}\left(\left(\sum_{k=1}^{4}\left(a_{t k}^{u}\right)^{2}+\sum_{k=1}^{4}\left(a_{t k}^{l}\right)^{2}\right) \cdot\left(\sum_{k=1}^{2}\left(h_{k}\left(A_{t}^{u}\right)\right)^{2}+\sum_{k=1}^{2}\left(h_{k}\left(A_{t}^{l}\right)\right)^{2}\right)\right)}$,

The bi-directional projection measure of IT2FNs reflects not only the distance and the angle between $A_{1}$ and $A_{2}$ but also the bi-directional projection magnitudes between $A_{1}$ and $A_{2}$. Clearly, the greater the value of $\operatorname{Bpoj}\left(A_{1}, A_{2}\right)$ is, the closer $A_{1}$ and $A_{2}$ are. The bi-directional projection measure is a normalized measure, i.e., $0 \leq \operatorname{Bpoj}\left(A_{1}, A_{2}\right) \leq 1$.

To explain the difference between the developed projection measure of IT2FNs and the developed bi-directional projection measure of IT2FNs, we provide the following example.

Example 2. Consider the following IT2FNs: $A_{1}=((0.4,0.5,0.5,0.8 ; 0.9,0.9),(0.5,0.7,0.7,0.8 ; 1,1))$ and $A_{2}=A_{3}=((0.3,0.5,0.5,0.7 ; 0.9,0.9),(0.4,0.6,0.6,0.7 ; 1,1))$.

By Eq. (35), we obtain that $\operatorname{Poj}_{A_{3}}\left(A_{1}\right)=3.3792$ and $\operatorname{Poj}_{A_{3}}\left(A_{2}\right)=2.9781$. In this situation, because $\operatorname{Poj}_{A_{3}}\left(A_{1}\right)>\operatorname{Poj}_{A_{3}}\left(A_{2}\right)$, we conclude that $A_{1}$ is closer to $A_{3}$ than $A_{2}$, which contradicts that $A_{1} \neq A_{2}=A_{3}$. From Eq. (36), we obtain that $\operatorname{Bpoj}\left(A_{1}, A_{3}\right)=0.9893$ and $\operatorname{Bpoj}\left(A_{2}, A_{3}\right)=1$, agreeing with $A_{1} \neq A_{2}=A_{3}$. This example illustrates that the projection measure of IT2FNs is not always viable in some situations, whereas the bi-directional projection measure of IT2FNs is viable and useful. Thus, the developed bi-directional projection measure of IT2FNs is better than the projection measure of IT2FNs to handle decision-making problems. 


\subsection{The models for deriving the optimal fuzzy measures}

Without loss of generality, in a MADM problem, it can be assumed that $P=\left\{p_{1}, p_{2}, \ldots, p_{m}\right\}$ is a collection of $m$ alternatives, $Z=\left\{z_{1}, z_{2}, \ldots, z_{n}\right\}$ is a collection of $n$ attributes, and $\Lambda=\left[A_{k j}\right]_{m \times n}$ is the decision matrix, where $A_{k j}=\left(\left(a_{k j}^{u}, a_{k j 2}^{u}, a_{k j}^{u}, a_{k j}^{u} ; h_{1}\left(A_{k j}^{u}\right), h_{2}\left(A_{k j}^{u}\right)\right),\left(a_{k j}^{l}, a_{k j 2}^{l}, a_{k j}^{l}, a_{k j}^{l} ; h_{1}\left(A_{k j}^{l}\right), h_{2}\left(A_{k j}^{l}\right)\right)\right)$ $(k=1,2, \ldots, m ; j=1,2, \ldots, n)$ is an IT2FN that represents how attribute $z_{j}$ is verified by alternative $p_{k}$. To handle the cases where the attributes' weights are partly known, this section constructs two models for deriving the optimal FMs and 2AFMs on the attributes set based on the developed bi-directional projection measure of IT2FNs, respectively.

It is assumed that $\Lambda^{+}=\left(A_{1}^{+}, A_{2}^{+}, \ldots, A_{n}^{+}\right)$and $\Lambda^{-}=\left(A_{1}^{-}, A_{2}^{-}, \ldots, A_{n}^{-}\right)$represent the positive and negative ideal alternatives, respectively, i.e.:

$$
\begin{aligned}
A_{j}^{+}= & \left(\left(a_{j 1}^{u+}, a_{j 2}^{u+}, a_{j 3}^{u+}, a_{j 4}^{u+} ; h_{1}\left(A_{j}^{u+}\right), h_{2}\left(A_{j}^{u+}\right)\right),\left(a_{j 1}^{l+}, a_{j 2}^{l+}, a_{j 3}^{l+}, a_{j 4}^{l+} ; h_{1}\left(A_{j}^{l+}\right), h_{2}\left(A_{j}^{l+}\right)\right)\right) \\
= & \left(\max _{k}\left(a_{k j}^{u}\right), \max _{k}\left(a_{k j 2}^{u}\right), \max _{k}\left(a_{k j 3}^{u}\right), \max _{k}\left(a_{k j 4}^{u}\right) ; \max _{k}\left(h_{1}\left(A_{k j}^{u}\right)\right), \max _{k}\left(h_{2}\left(A_{k j}^{u}\right)\right)\right), \\
& \left.\left(\max _{k}\left(a_{k j 1}^{l}\right), \max _{k}\left(a_{k j 2}^{l}\right), \max _{k}\left(a_{k j 3}^{l}\right), \max _{k}\left(a_{k j}^{l}\right) ; \max _{k}\left(h_{1}\left(A_{k j}^{l}\right)\right), \max _{k}\left(h_{2}\left(A_{k j}^{l}\right)\right)\right)\right)(j=1,2, \ldots, n), \\
A_{j}^{-}= & \left(\left(a_{j 1}^{u-}, a_{j 2}^{u-}, a_{j 3}^{u-}, a_{j 4}^{u-} ; h_{1}\left(A_{j}^{u-}\right), h_{2}\left(A_{j}^{u-}\right)\right),\left(a_{j 1}^{l-}, a_{j 2}^{l-}, a_{j 3}^{l-}, a_{j 4}^{l-} ; h_{1}\left(A_{j}^{l-}\right), h_{2}\left(A_{j}^{l-}\right)\right)\right) \\
= & \left(\left(\min _{k}\left(a_{k j 1}^{u}\right), \min _{k}\left(a_{k j 2}^{u}\right), \min _{k}\left(a_{k j 3}^{u}\right), \min _{k}\left(a_{k j 4}^{u}\right) ; \min _{k}\left(h_{1}\left(A_{k j}^{u}\right)\right), \min _{k}\left(h_{2}\left(A_{k j}^{u}\right)\right)\right),\right. \\
& \left.\left(\min _{k}\left(a_{k j 1}^{l}\right), \min _{k}\left(a_{k j 2}^{l}\right), \min _{k}\left(a_{k j 3}^{l}\right), \min _{k}\left(a_{k j 4}^{l}\right) ; \min _{k}\left(h_{1}\left(A_{k j}^{l}\right)\right), \min _{k}\left(h_{2}\left(A_{k j}^{l}\right)\right)\right)\right)(j=1,2, \ldots, n) .
\end{aligned}
$$

Then, according to the subtraction operation of trapezoidal IT2FNs [5], we compute the difference between $A_{j}^{+}$and $A_{j}^{-}$, the difference between $A_{j}^{+}$and $A_{k j}$, and the difference between $A_{k j}$ and $A_{j}^{-}:$

$$
\begin{aligned}
A_{j}^{+} \Theta A_{j}^{-}= & \left(\left(a_{j 1}^{u+}-a_{j 4}^{u-}, a_{j 2}^{u+}-a_{j 3}^{u-}, a_{j 3}^{u+}-a_{j 2}^{u-}, a_{j 4}^{u+}-a_{j 1}^{u-} ; \min \left(h_{1}\left(A_{j}^{u+}\right), h_{1}\left(A_{j}^{u-}\right)\right), \min \left(h_{2}\left(A_{j}^{u+}\right), h_{2}\left(A_{j}^{u-}\right)\right)\right),\right. \\
& \left.\left(a_{j 1}^{l+}-a_{j 4}^{l-}, a_{j 2}^{l+}-a_{j 3}^{l-}, a_{j 3}^{l+}-a_{j 2}^{l-}, a_{j 4}^{l+}-a_{j 1}^{l-} ; \min \left(h_{1}\left(A_{j}^{l+}\right), h_{1}\left(A_{j}^{l-}\right)\right), \min \left(h_{2}\left(A_{j}^{l-}\right), h_{2}\left(A_{j}^{l-}\right)\right)\right)\right), \\
A_{j}^{+} \Theta A_{k j}= & \left(\left(a_{j 1}^{u+}-a_{k j 4}^{u}, a_{j 2}^{u+}-a_{k j 3}^{u}, a_{j 3}^{u+}-a_{k j 2}^{u}, a_{j 4}^{u+}-a_{k j 1}^{u} ; \min \left(h_{1}\left(A_{j}^{u+}\right), h_{1}\left(A_{k j}^{u}\right)\right), \min \left(h_{2}\left(A_{j}^{u+}\right), h_{2}\left(A_{k j}^{u}\right)\right)\right),\right. \\
& \left.\left(a_{j 1}^{l+}-a_{k j 4}^{l}, a_{j 2}^{l+}-a_{k j 3}^{l}, a_{j 3}^{l+}-a_{k j 2}^{l}, a_{j 4}^{l+}-a_{k j 1}^{l} ; \min \left(h_{1}\left(A_{j}^{l+}\right), h_{1}\left(A_{k j}^{l}\right)\right), \min \left(h_{2}\left(A_{j}^{l+}\right), h_{2}\left(A_{k j}^{l}\right)\right)\right)\right), \\
A_{k j} \Theta A_{j}^{-}= & \left(a_{k j 1}^{u}-a_{j 4}^{u-}, a_{k j 2}^{u}-a_{j 3}^{u-}, a_{k j 3}^{u}-a_{j 2}^{u-}, a_{k j 4}^{u}-a_{j 1}^{u-} ; \min \left(h_{1}\left(A_{k j}^{u}\right), h_{1}\left(A_{j}^{u-}\right)\right), \min \left(h_{2}\left(A_{k j}^{u}\right), h_{2}\left(A_{j}^{u-}\right)\right)\right), \\
& \left.\left(a_{k j 1}^{l}-a_{j 4}^{l-}, a_{k j 2}^{l}-a_{j 3}^{l-}, a_{k j 3}^{l}-a_{j 2}^{l-}, a_{k j 4}^{l}-a_{j 1}^{l-} ; \min \left(h_{1}\left(A_{k j}^{l}\right), h_{1}\left(A_{j}^{l-}\right)\right), \min \left(h_{2}\left(A_{k j}^{l}\right), h_{2}\left(A_{j}^{l-}\right)\right)\right)\right) .
\end{aligned}
$$

In addition, by Eq. (36), we obtain the bi-directional projection between $A_{j}^{+} \Theta A_{j}^{-}$and $A_{i j} \Theta A_{j}^{-}$, and the bi-directional projection between $A_{j}^{+} \Theta A_{j}^{-}$and $A_{j}^{+} \Theta A_{i j}$ :

$$
\begin{gathered}
\operatorname{Bpoj}\left(A_{j}^{+} \Theta A_{j}^{-}, A_{k j} \Theta A_{j}^{-}\right)=\frac{2\left(A_{j}^{+} \Theta A_{j}^{-}\right) \cdot\left(A_{k j} \Theta A_{j}^{-}\right)}{\left|A_{j}^{+} \Theta A_{j}^{-}\right|^{2}+\left|A_{k j} \Theta A_{j}^{-}\right|^{2}}, \\
\operatorname{Bpoj}\left(A_{j}^{+} \Theta A_{j}^{-}, A_{j}^{+} \Theta A_{k j}\right)=\frac{2\left(A_{j}^{+} \Theta A_{j}^{-}\right) \cdot\left(A_{j}^{+} \Theta A_{k j}\right)}{\left|A_{j}^{+} \Theta A_{j}^{-}\right|^{2}+\left|A_{j}^{+} \Theta A_{k j}\right|^{2}} .
\end{gathered}
$$

Next, based on the TOPSIS methodology [17], the closeness degree between 
$\operatorname{Bpoj}\left(A_{j}^{+} \Theta A_{j}^{-}, A_{k j} \Theta A_{j}^{-}\right)$and $\operatorname{Bpoj}\left(A_{j}^{+} \Theta A_{j}^{-}, A_{j}^{+} \Theta A_{k j}\right)$ is obtained:

$$
\frac{\operatorname{Bpoj}\left(A_{j}^{+} \Theta A_{j}^{-}, A_{k j} \Theta A_{j}^{-}\right)}{\operatorname{Bpoj}\left(A_{j}^{+} \Theta A_{j}^{-}, A_{k j} \Theta A_{j}^{-}\right)+\operatorname{Bpoj}\left(A_{j}^{+} \Theta A_{j}^{-}, A_{j}^{+} \Theta A_{k j}\right)} .
$$

To achieve the optimal FMs on the attributes set $Z$, the following model is solved:

$$
\begin{aligned}
\max \sum_{k=1}^{m} \sum_{j=1}^{n} \frac{\operatorname{Bpoj}\left(A_{j}^{+} \Theta A_{j}^{-}, A_{k j} \Theta A_{j}^{-}\right)}{\operatorname{Bpoj}\left(A_{j}^{+} \Theta A_{j}^{-}, A_{k j} \Theta A_{j}^{-}\right)+B p o j\left(A_{j}^{+} \Theta A_{j}^{-}, A_{j}^{+} \Theta A_{k j}\right)} \psi\left(\left\{z_{j}\right\}\right) \\
\text { s.t.. }\left\{\begin{array}{l}
\mu\left(\left\{z_{j}\right\}\right) \in H_{z_{j}}, j=1,2, \ldots, n ; \\
\mu(\phi)=0, \mu(Z)=1 ; \\
\mu(B) \leq \mu(T), \forall S, T \subset Z, B \subset T .
\end{array}\right.
\end{aligned}
$$

where $H_{z_{j}}$ is the range of known weights information of attribute $z_{j}(j=1,2, \ldots, n)$. Once model (40) is solved, using Eq. (22), the generalized Banzhaf values of the attributes are derived and regarded as the attributes' weights.

Using Eq. (28) and Theorem 4, model (40) can be equivalently converted into:

$$
\begin{aligned}
& \max \frac{3-n}{2} \sum_{k=1}^{m} \sum_{j=1}^{n} \frac{\operatorname{Bpoj}\left(A_{j}^{+} \Theta A_{j}^{-}, A_{k j} \Theta A_{j}^{-}\right)}{\operatorname{Bpoj}\left(A_{j}^{+} \Theta A_{j}^{-}, A_{k j} \Theta A_{j}^{-}\right)+\operatorname{Bpoj}\left(A_{j}^{+} \Theta A_{j}^{-}, A_{j}^{+} \Theta A_{k j}\right)} \psi\left(\left\{z_{j}\right\}\right)+ \\
& \sum_{k=1}^{m} \sum_{j=1}^{n} \frac{\operatorname{Bpoj}\left(A_{j}^{+} \Theta A_{j}^{-}, A_{k j} \Theta A_{j}^{-}\right)}{\operatorname{Bpoj}\left(A_{j}^{+} \Theta A_{j}^{-}, A_{k j} \Theta A_{j}^{-}\right)+B \operatorname{Bpoj}\left(A_{j}^{+} \Theta A_{j}^{-}, A_{j}^{+} \Theta A_{k j}\right)}\left(\sum_{z_{t} \in Z \backslash z_{j}}\left(\psi\left(\left\{z_{t}, z_{j}\right\}\right)-\psi\left(\left\{z_{t}\right\}\right)\right)\right) \\
& \qquad . t . .\left\{\begin{array}{l}
\mu\left(\left\{z_{j}\right\}\right) \in H_{z_{j}}, j=1,2, \ldots, n ; \\
\mu\left(\left\{z_{j}\right\}\right) \geq 0, j=1,2, \ldots, n ; \\
\sum_{\left\{z_{t}, z_{j}\right\} \subset Z} \mu\left(\left\{z_{t}, z_{j}\right\}\right)-(|Z|-2) \sum_{z_{j} \in Z} \mu\left(\left\{z_{j}\right\}\right)=1 ; \\
\sum_{\left.\left\{z_{t}\right\} \in B \mid z_{j}\right\}}\left(\mu\left(\left\{z_{t}, z_{j}\right\}\right)-\mu\left(\left\{z_{t}\right\}\right)\right) \geq(|B|-2) \mu\left(\left\{z_{j}\right\}\right), \forall B \subset Z, \forall z_{j} \in B,|B| \geq 2 .
\end{array}\right.
\end{aligned}
$$

Once model (41) is solved, using Eq. (28), the Banzhaf values with 2AFMs of the attributes are derived and regarded as the attributes' weights.

\section{The created approaches to interval type-2 fuzzy MADM problems}

In what follows, based on the proposed operators and constructed linear models, two novel MADM approaches for handling IT2F decision-making problems with interactive attributes and incomplete weights information are presented.

\section{1) The first MADM approach based on the BIT2FAC operator}

Step 1: Evaluate the alternatives $p_{k}(k=1,2, \cdots, m)$ on the attributes $z_{j}(j=1,2, \cdots, n)$, and build the IT2F decision matrix $\Lambda=\left[A_{k j}\right]_{m \times n}$.

Step 2: If the criteria weighting vector is partly unknown, use model (40) to derive the optimal FMs $\mu(B)$ on the attributes set $B \subset Z$; otherwise, go to step 3 .

Step 3: Compute the generalized Banzhaf value $\psi(B)$ with FM $\mu(B)$ on the attributes set $B$ using Eq. (22).

Step 4: Calculate the comprehensive attribute value $A_{k}$ of alternative $p_{k}(k=1,2, \ldots, m)$ using the 
proposed BIT2FAC operator with Eq. (25).

Step 5: Compute the combined ranking values $R\left(A_{k}\right)$ of the comprehensive attribute values $A_{k}(k=1,2, \ldots, m)$ using Eq. (5).

Step 6: Apply Definition 4 to derive the ranking of the alternatives $p_{1}, p_{2}, \ldots, p_{m}$ and select the optimal alternative. The larger the ranking value $R\left(A_{k}\right)$, the better the alternative $p_{k}(k=1,2, \ldots, m)$.

2) The second MADM approach based on the 2ABIT2FAC operator

Step 1: Evaluate the alternatives $p_{k}(k=1,2, \cdots, m)$ on the attributes $z_{j}(j=1,2, \cdots, n)$, and build the IT2F decision matrix $\Lambda=\left[A_{k j}\right]_{m \times n}$.

Step 2: If the criteria weighting vector is partly unknown, use model (41) to derive the optimal 2AFMs $\mu(B)$ on the attributes set $B \subset Z$; otherwise, go to step 3 .

Step 3: Compute the Banzhaf value $\psi\left(\left\{z_{j}\right\}\right)$ with $2 \mathrm{AFM} \mu\left(\left\{z_{j}\right\}\right)$ on the attribute $z_{j}$ using Eq. (28).

Step 4: Calculate the comprehensive attributes value $A_{k}$ of alternative $p_{k}(k=1,2, \ldots, m)$ using the proposed 2ABIT2FAC operator with Eq. (31).

Step 5: Compute the combined ranking values $R\left(A_{k}\right)$ of the comprehensive attribute values $A_{k}(k=1,2, \ldots, m)$ using Eq. (5).

Step 6: Apply Definition 4 to derive the ranking of the alternatives $p_{1}, p_{2}, \ldots, p_{m}$ and select the optimal alternative.

Although the two created MADM approaches can handle IT2F decision-making problems, some differences exist between them.

1) Regarding computational complexity, the first MADM approach is based on FM, which requires $2^{n}$ coefficients; however, the second MADM approach is based on 2AFM, which only needs $n \times(n+1) / 2$ coefficients. Therefore, the computational complexity of the second MADM approach is lower, and it is more practical than the first one.

2) To reflect the interactions of the attributes, the first MADM approach is based on a generalized Banzhaf index with Eq. (22), which globally considers the interactions of the attributes, whereas the second MADM approach is based on the Banzhaf function with 2AFM with Eq. (28), which only reflects the interrelations between any two attributes. Consequently, the first MADM approach can reflect the interactions of the attributes more comprehensively than the second one.

In real MADM environments, when decision-makers (DMs) can endure a long computational time period and expect to globally reflect the interactions of the attributes, the first MADM approach is the best choice. Otherwise, the second MADM approach is the optimal choice.

\section{Application examples}

In this section, an illustrative case is offered to present the application of the created approaches to the service quality evaluation of commercial bank problems. Additionally, a comparative study with 
existing methods [13] [14] [18] is carried out to illustrate the superiority of the created approaches.

\subsection{Application of the created MADM approaches}

Example 3. To better understand the service quality of commercial banks in China, four famous commercial banks are considered for assessment, anonymously denoted as $p_{1}, p_{2}, p_{3}$ and $p_{4}$. Eight attributes of service quality are identified by reviewing the relevant literature [36] [46] [48], and they are:

$z_{1}$ : Technology level, which assesses the hardware equipment for banking business, the application level of science and technology, the networking level of the bank and the speed of technological renewal;

$z_{2}$ : Service attitude, which assesses the warm greetings of customers by staff and the courtesy of staff to customers;

$z_{3}$ : Consultation service, which assesses the number of staff in the lobby for consulting service, the level of business proficiency, the status of the job, the scope of the consulting business and the implementation of consulting services;

$z_{4}$ : Waiting area environment, which assesses the waiting area, the decoration status, the lighting, the temperature control, the number of seats and their comfort, the wireless network service, the electronic information display and the voice prompts;

$z_{5}$ : Window opening situation, which assesses the number of service opening windows and waiting time of customers;

$z_{6}:$ Operational capacity, which assesses the skills of business people, the proficiency in business, the efficiency of work, the error rate and the customer satisfaction;

$z_{7}$ : Security, which assesses the soundness of security monitoring equipment, the provision of security personnel, the responsibility of security personnel to perform their duties, the anti-information leakage, the anti-copy and the anti-theft brush technology;

$z_{8}$ : Financial product, which assesses the richness and the management of financial management varieties, the interest rate of property products and the safety of financial products.

The seven IT2FNs linguistic terms below are utilized to assess the service quality of the four commercial banks on the above eight attributes (Table 2). The decision matrix is shown in Table 3.

Table 2. Linguistic ratings and their corresponding trapezoid IT2FNs

\begin{tabular}{lc}
\hline Linguistic Terms & Trapezoid IT2FNs \\
\hline Extremely Poor (EP) & $((0,0,0,0.1 ; 1,1),(0,0,0,0.05 ; 0.9,0.9))$ \\
Poor (P) & $((0,0.1,0.15,0.3 ; 1,1),(0.05,0.1,0.1,0.2 ; 0.9,0.9))$ \\
Slightly Poor (SP) & $((0.15,0.3,0.35,0.5 ; 1,1),(0.2,0.25,0.3,0.4 ; 0.9,0.9))$ \\
Fair (F) & $((0.3,0.5,0.55,0.7 ; 1,1),(0.4,0.45,0.5,0.6 ; 0.9,0.9))$ \\
Slightly Good (SG) & $((0.5,0.7,0.75,0.9 ; 1,1),(0.6,0.65,0.7,0.85 ; 0.9,0.9))$ \\
Good (G) & $((0.7,0.9,0.95,1 ; 1,1),(0.8,0.85,0.9,0.95 ; 0.9,0.9))$ \\
Extremely Good (EG) & $((0.9,1,1,1 ; 1,1),(0.95,1,1,1 ; 0.9,0.9))$ \\
\hline
\end{tabular}


Table 3. The decision matrix

\begin{tabular}{ccccccccc}
\hline & $z_{1}$ & $z_{2}$ & $z_{3}$ & $z_{4}$ & $z_{5}$ & $z_{6}$ & $z_{7}$ \\
\hline$p_{1}$ & SG & F & F & G & F & SP & SP & SG \\
$p_{2}$ & SP & EG & SP & F & EG & EG & SP & F \\
$p_{3}$ & F & G & SP & SG & G & G & SP & F \\
$p_{4}$ & G & EG & MG & F & SP & EG & G \\
\hline
\end{tabular}

Using the first MADM approach, based on the BIT2FAC operator, $2^{8}$ coefficients are to be determined, while with the second MADM approach, based on the 2 ABIT2FAC operator, $(8 \times 9) / 2$ coefficients are needed. Therefore, we select the second MADM approach to solve Example 3 and its calculation procedure are given below:

[Step 1] It is assumed that the attributes are independent, and the following importance values of attributes $z_{j}(j=1,2, \ldots, 8)$ are provided by experts:

$$
\mu\left(\left\{z_{1}\right\}\right)=\mu\left(\left\{z_{2}\right\}\right)=\mu\left(\left\{z_{3}\right\}\right)=\mu\left(\left\{z_{4}\right\}\right)=\mu\left(\left\{z_{8}\right\}\right)=0.1, \mu\left(\left\{z_{5}\right\}\right)=\mu\left(\left\{z_{7}\right\}\right)=0.15, \mu\left(\left\{z_{6}\right\}\right)=0.2 .
$$

Because all the attributes are independent, according to the property of additive measure, we obtain $\mu\left(\left\{z_{1}, z_{2}\right\}\right)=\mu\left(\left\{z_{1}\right\}\right)+\mu\left(\left\{z_{2}\right\}\right)=0.2$. Similarly, the other 2AFMs $\mu(B)$ on the attribute set $B \subset Z$ are obtained:

$$
\begin{aligned}
& \mu\left(\left\{z_{1}, z_{3}\right\}\right)=\mu\left(\left\{z_{1}, z_{4}\right\}\right)=\mu\left(\left\{z_{1}, z_{8}\right\}\right)=\mu\left(\left\{z_{2}, z_{3}\right\}\right)=\mu\left(\left\{z_{2}, z_{4}\right\}\right)=\mu\left(\left\{z_{2}, z_{8}\right\}\right)=\mu\left(\left\{z_{3}, z_{4}\right\}\right)=0.2, \\
& \left.\left\{z_{3}, z_{8}\right\}\right)=\mu\left(\left\{z_{4}, z_{5}\right\}=\mu\left(\left\{z_{4}, z_{8}\right\}\right)=0.2, \mu\left(\left\{z_{1}, z_{5}\right\}\right)=\mu\left(\left\{z_{1}, z_{7}\right\}\right)=\mu\left(\left\{z_{2}, z_{5}\right\}\right)=\mu\left(\left\{z_{2}, z_{7}\right\}\right)=0.25,\right. \\
& \mu\left(\left\{z_{3}, z_{5}\right\}\right)=\mu\left(\left\{z_{3}, z_{7}\right\}\right)=\mu\left(\left\{z_{4}, z_{7}\right\}\right)=\mu\left(\left\{z_{5}, z_{8}\right\}\right)=\mu\left(\left\{z_{7}, z_{8}\right\}\right)=0.25, \mu\left(\left\{z_{1}, z_{6}\right\}\right)=0.3, \\
& \mu\left(\left\{z_{2}, z_{6}\right\}\right)=\mu\left(\left\{z_{3}, z_{6}\right\}\right)=\mu\left(\left\{z_{4}, z_{6}\right\}\right)=\mu\left(\left\{z_{5}, z_{7}\right\}\right) \mu\left(\left\{z_{6}, z_{8}\right\}\right)=0.3, \mu\left(\left\{z_{5}, z_{6}\right\}\right)=\mu\left(\left\{z_{6}, z_{7}\right\}\right)=0.35 .
\end{aligned}
$$

[Step 2] Eq. (28) is used to compute the Banzhaf value $\psi\left(\left\{z_{j}\right\}\right)$ with $2 \mathrm{AFM} \mu\left(\left\{z_{j}\right\}\right)$ on the attribute $z_{j}(j=1,2, \ldots, 8)$. An example is illustrated below:

$$
\begin{aligned}
& \psi\left(\left\{z_{1}\right\}\right)=-\frac{5}{2} \mu\left(\left\{z_{1}\right\}\right)+\sum_{z_{i} \in Z \backslash z_{1}} \frac{1}{2}\left(\mu\left(\left\{z_{1}, z_{i}\right\}\right)-\mu\left(\left\{z_{i}\right\}\right)\right) \\
= & -\frac{5}{2} \times 0.1+\frac{1}{2} \times(0.2-0.1+0.2-0.1+0.2-0.1+0.25-0.15+0.3-0.2+0.25-0.15+0.2-0.1)=0.1 .
\end{aligned}
$$

Similarly, it is obtained:

$$
\psi\left(\left\{z_{2}\right\}\right)=\psi\left(\left\{z_{3}\right\}\right)=\psi\left(\left\{z_{4}\right\}\right)=\psi\left(\left\{z_{8}\right\}\right)=0.1, \psi\left(\left\{z_{5}\right\}\right)=\psi\left(\left\{z_{7}\right\}\right)=0.15, \psi\left(\left\{z_{6}\right\}\right)=0.2 .
$$

[Step 3] The proposed 2ABIT2FAC operator (algebraic) with Eq. (31) is used to derive the comprehensive attribute values $A_{k}$ of the alternatives $p_{k}(k=1,2,3,4)$. The detailed computation of $A_{1}$ is shown below:

$$
\begin{aligned}
& A_{1}=2 \text { ABIT } 2 \text { FAC }\left(A_{11}, A_{12}, \ldots, A_{18}\right)=\bigotimes_{j=1}^{8} A_{1 j} \psi\left(z_{j}\right) \\
= & \left(\left(\prod_{j=1}^{8}\left(a_{1 j 1}^{u}\right)^{\psi\left(z_{j}\right)}, \prod_{j=1}^{8}\left(a_{1 j 2}^{u}\right)^{\psi\left(z_{j}\right)}, \prod_{j=1}^{8}\left(a_{1 j 3}^{u}\right)^{\psi\left(z_{j}\right)}, \prod_{j=1}^{8}\left(a_{1 j 4}^{u}\right)^{\psi\left(z_{j}\right)} ; \prod_{j=1}^{8}\left(h_{1}\left(A_{1 j}^{u}\right)\right)^{\psi\left(z_{j}\right)}, \prod_{j=1}^{8}\left(h_{2}\left(A_{1 j}^{u}\right)\right)^{\psi\left(z_{j}\right)}\right),\right. \\
& \left.\left(\prod_{j=1}^{8}\left(a_{1 j 1}^{l}\right)^{\psi\left(z_{j}\right)}, \prod_{j=1}^{8}\left(a_{1 j 2}^{l}\right)^{\psi\left(z_{j}\right)}, \prod_{j=1}^{8}\left(a_{1 j 3}^{l}\right)^{\psi\left(z_{j}\right)}, \prod_{j=1}^{8}\left(a_{1 j 4}^{l}\right)^{\psi\left(z_{j}\right)} ; \prod_{j=1}^{8}\left(h_{1}\left(A_{1 j}^{l}\right)\right)^{\psi\left(z_{j}\right)}, \prod_{j=1}^{8}\left(h_{2}\left(A_{1 j}^{l}\right)\right)^{\psi\left(z_{j}\right)}\right)\right) \\
= & ((0.2837,0.4743,0.5276,0.6781 ; 1,1),(0.3648,0.4202,0.4743,0.5844,0.9,0.9)) .
\end{aligned}
$$

Similarly: it is obtained: 


$$
\begin{aligned}
& A_{2}=((0.3859,0.5712,0.6145,0.7306 ; 1,1),(0.4632,0.5247,0.5712,0.6552 ; 0.9,0.9)), \\
& A_{3}=((0.3887,0.5929,0.6480,0.7748 ; 1,1),(0.4785,0.5366,0.5929,0.6903 ; 0.9,0.9)), \\
& A_{4}=((0.4562,0.6490,0.6950,0.8029 ; 1,1),(0.5400,0.6004,0.6490,0.7340 ; 0.9,0.9)) .
\end{aligned}
$$

[Step 4] Eq. (5) is used to compute the combined ranking value $R\left(A_{k}\right)$ of the comprehensive attribute value $A_{k}(k=1,2,3,4)$ :

$$
R\left(A_{1}\right)=0.6578, R\left(A_{2}\right)=0.8342, R\left(A_{3}\right)=0.8792, R\left(A_{4}\right)=0.9952 .
$$

[Step 5] By Definition 4, we find that the final ranking of commercial banks is $p_{4}>p_{3}>p_{2}>p_{1}$, and $p_{4}$ is the best commercial bank.

\subsection{Discussion about some special cases}

Below we analyse the effects of four special cases of ATs on the final ranking values and on the corresponding ranking alternatives. As we can see from Table 4 ( $\gamma=2$ in the Frank and Aczel-Alsina operations), the ranking values by different operations are different; however, the preferred ordering of the commercial banks are identical in the four cases.

Table 4. Ranking results in light of four special cases of AT

\begin{tabular}{ccc}
\hline Operations & Ranking values of $p_{k}(k=1,2,3,4)$ & Preferred order \\
\hline Algebraic operations & $R\left(A_{1}\right)=0.6578, R\left(A_{2}\right)=0.8342, R\left(A_{3}\right)=0.8792, R\left(A_{4}\right)=0.9952$. & $p_{4}>p_{3}>p_{2}>p_{1}$ \\
Einstein operations & $R\left(A_{1}\right)=0.6786, R\left(A_{2}\right)=0.9051, R\left(A_{3}\right)=0.9256, R\left(A_{4}\right)=1.0653$. & $p_{4}>p_{3}>p_{2}>p_{1}$ \\
Frank operations & $R\left(A_{1}\right)=0.6676, R\left(A_{2}\right)=0.8649, R\left(A_{3}\right)=0.9002, R\left(A_{4}\right)=1.0261$. & $p_{4}>p_{3}>p_{2}>p_{1}$ \\
Aczel-Alsina operations & $R\left(A_{1}\right)=0.5825, R\left(A_{2}\right)=0.6316, R\left(A_{3}\right)=0.7057, R\left(A_{4}\right)=0.7441$. & $p_{4}>p_{3}>p_{2}>p_{1}$ \\
\hline
\end{tabular}

Further, we discuss the effects of the parameter $\gamma$ on the preferred ordering of this example. We use the Frank and Aczel-Alsina operations to illustrate the effects. The ranking results for different values of $\gamma$ from Frank and Aczel-Alsina operations are depicted in Figs. 2 and 3. The following conclusions are drawn:

1) The ranking values derived by the created approach based on Frank operations are not lower than those derived by the created approach based on Aczel-Alsina operations for the same value of the parameter $\gamma$, and their deviations become larger as the value of parameter $\gamma$ increases.

2) The ranking values derived by the created approach based on Frank operations increase as parameter $\gamma$ increases (for the same alternative). However, the ranking values derived by the created approach based on Aczel-Alsina operations decrease as parameter $\gamma$ increases (for the same alternative).

3) For the Frank and Aczel-Alsina operations, the same preferred ordering of alternatives is obtained for different values of parameter $\gamma$. This denotes that the created approach verifies the isotonicity property.

4) We could consider the parameter $\gamma$ as the "DMs' attitude". For the Frank operations, the larger the value of parameter $\gamma$ becomes, the more optimistic the DMs are; the smaller the value of $\gamma$ becomes, the more pessimistic the DMs are. However, for the Aczel-Alsina 
operations, the inverse interpretation applies.

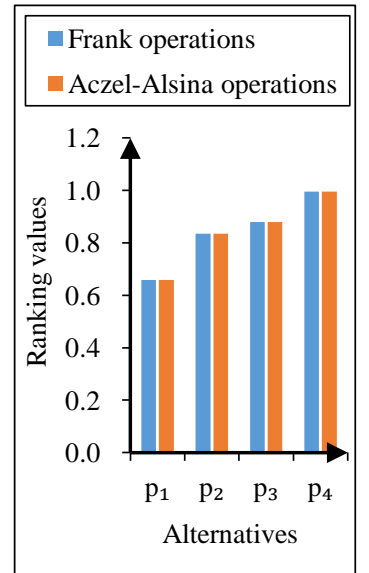

(1) $\gamma=1$

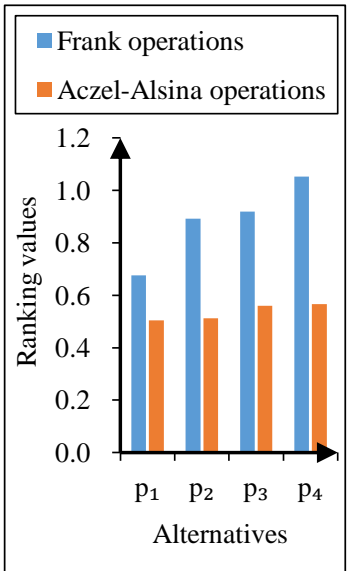

(5) $\gamma=5$

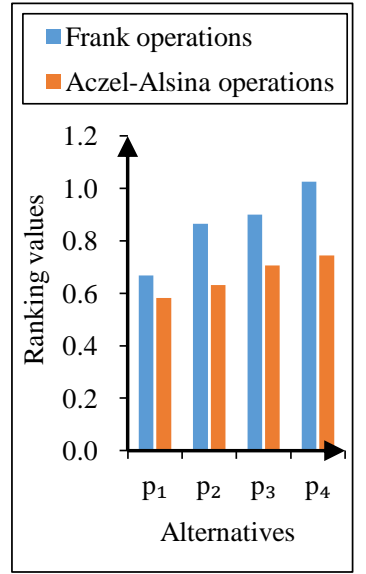

(2) $\gamma=2$

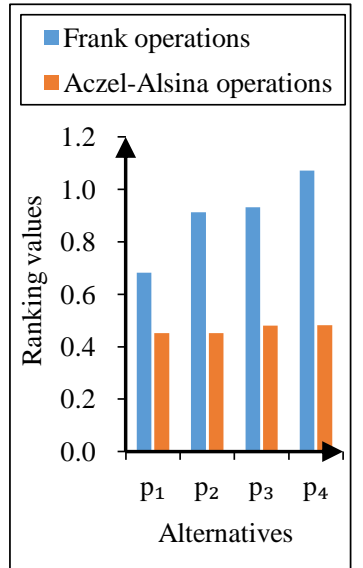

(6) $\gamma=7$

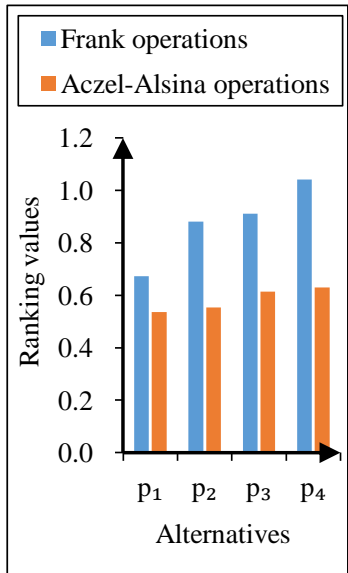

(3) $\gamma=3$

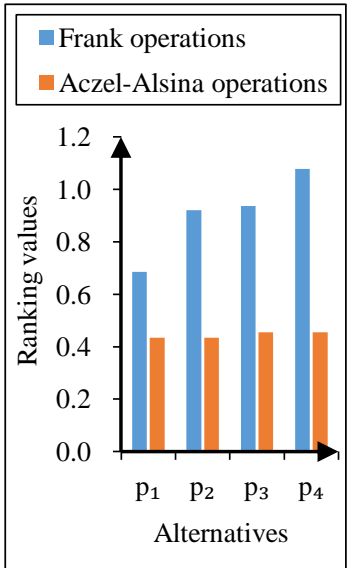

(7) $\gamma=9$

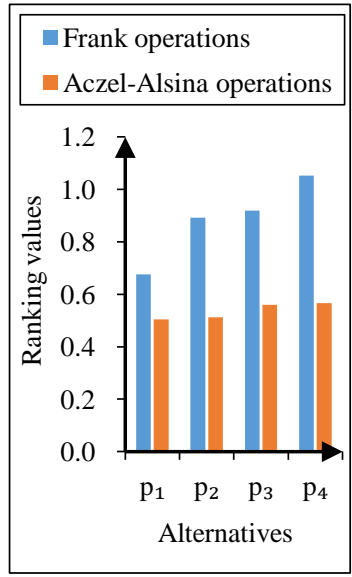

(4) $\gamma=4$

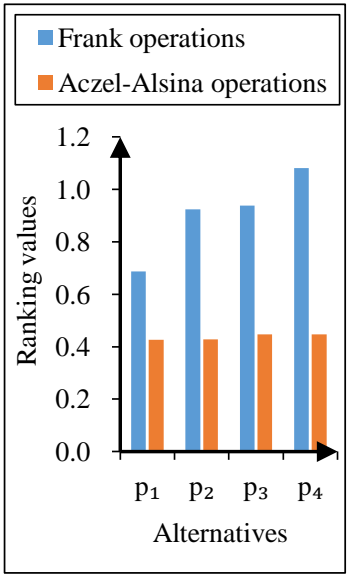

(8) $\gamma=10$

Fig. 2. Ranking results of the alternatives with different values of $\gamma$

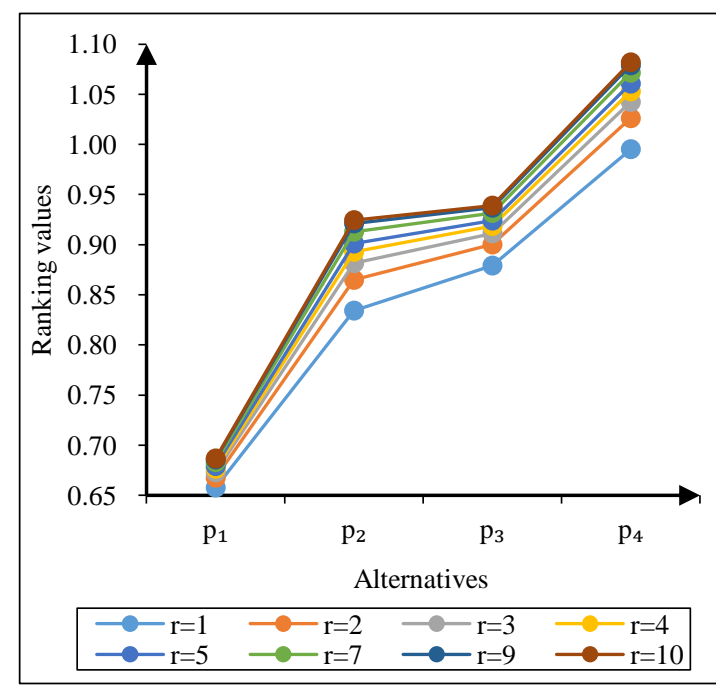

(1) Frank operations

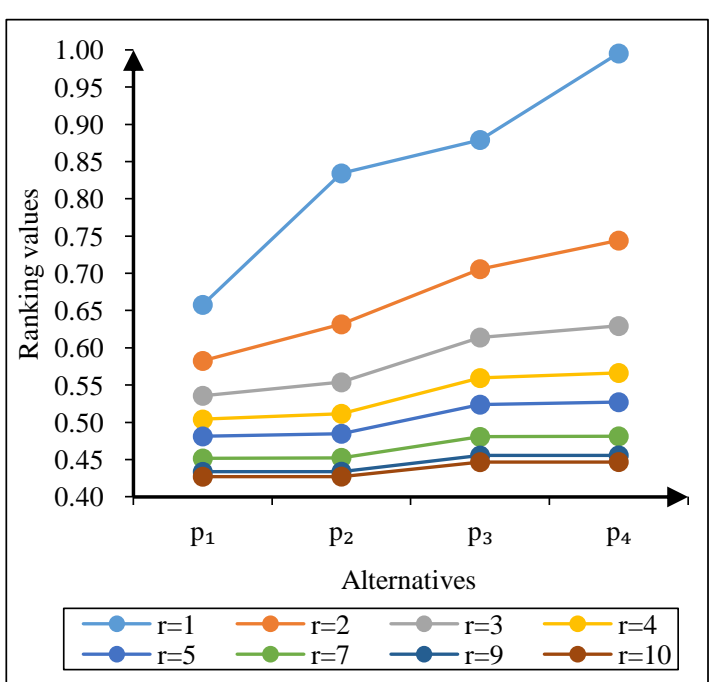

(2) Aczel-Alsina operations

Fig. 3. Ranking results of the alternatives with different operations

\subsection{Validity test of the created approach}

The different MADM approaches might provide different ranking results for the same 
decision-making problem. Therefore, the following test criteria provided by Wang and Triantaphyllou [45] is applied to analyse the reliability and validity of the created MADM approach.

Test criterion 1. An effective MADM approach should not alter the optimal alternative when substituting a non-optimal alternative for another non-optimal alternative without changing the attributes' weights.

Using test criterion 1 , the worse alternative $p^{1}=\{\mathrm{F}, \mathrm{SP}, \mathrm{F}, \mathrm{G}, \mathrm{F}, \mathrm{SP}, \mathrm{SP}, \mathrm{F}\}$ is substituted for the non-optimal alternative $p_{1}=\{\mathrm{SG}, \mathrm{F}, \mathrm{F}, \mathrm{G}, \mathrm{F}, \mathrm{SP}, \mathrm{SP}, \mathrm{SG}\}$ in the initial decision matrix. Using the BIT2FAC operator with Frank operations and $\gamma=2$, in Step 3 to this adjusted data, the following comprehensive values of each commercial bank $p_{k}(k=1,2,3,4)$ are obtained:

$$
\begin{aligned}
& A_{1}=((0.2880,0.4799,0.5333,0.6828 ; 1,1),(0.3703,0.4258,0.4799,0.903 ; 0.9,0.9)), \\
& A_{2}=((0.4033,0.5870,0.6281,0.7386 ; 1,1),(0.4810,0.5427,0.5870,0.6668 ; 0.9,0.9)), \\
& A_{3}=((0.3983,0.6039,0.6589,0.7822 ; 1,1),(0.4898,0.5478,0.6039,0.6996 ; 0.9,0.9)), \\
& A_{4}=((0.4724,0.6638,0.7083,0.8110 ; 1,1),(0.5568,0.6169,0.6638,0.7453 ; 0.9,0.9)) .
\end{aligned}
$$

The combined ranking values of all commercial banks are:

$$
R\left(A_{1}\right)=0.5680, R\left(A_{2}\right)=0.8649, R\left(A_{3}\right)=0.9002, R\left(A_{4}\right)=1.0261 .
$$

Thus, the ranking result is $p_{4}>p_{3}>p_{2}>p_{1}$, i.e., the best commercial bank is again $p_{4}$. Therefore, the created MADM approach is shown to verify test criterion 1.

Test criterion 2. An effective MADM approach needs to verify transitive property.

Test criterion 3. When a MADM problem is decomposed into several sub-problems, and the same approach is used to handle these sub-problems to provide the ranking of the alternatives, the integrated ranking of the alternatives needs to be the same as the original MADM problem.

Using test criteria 2 and 3, we decompose the initial MADM problem into two smaller MADM problems $\left\{p_{1}, p_{2}, p_{4}\right\}$ and $\left\{p_{2}, p_{3}, p_{4}\right\}$. According to the procedure of the created MADM approach, we derive preferred orders $p_{4}>p_{2}>p_{1}$ and $p_{4}>p_{3}>p_{2}$ for the two sub-problems, respectively. If the orderings of the sub-problems are integrated together, we derive the integrated preferred order as $p_{4}>p_{3}>p_{2}>p_{1}$, which is the same as the ranking in the initial MADM problem and consistent with the transitive property. Therefore, the created MADM approach is shown to verify test criteria 2 and 3.

\subsection{Comparison with existing methods}

To further verify the effectiveness of the two created approaches, experimental results are compared with results from existing MADM approaches: Hu et al.'s approach [18] based on the weighted averaging operator of trapezoidal IT2FNs (TIT2-WAA) and maximizing deviation method; Gong et al.'s approach [13] based on the Einstein weighted geometric operator of trapezoidal IT2FNs (TIT2FEWG); and Gong et al.'s approach [14] based on the weighted geometric Bonferroni mean of trapezoidal IT2FNs (TIT2FWGB). It is assumed that $p=q=1$ for Gong et al.'s approach [14], and it is assumed that the operational laws are Frank operations and $\gamma=2$ for the created approach. The preferred orderings of the commercial banks in Example 3 for the different approaches are listed in Table 5. 
Table 5. Ranking results from different approaches for Example 3

\begin{tabular}{clc}
\hline Approaches & \multicolumn{1}{c}{ Ranking values (R) } & Preferred order \\
\hline Hu et al's approach [18] & $R\left(A_{1}\right)=-0.0071, R\left(A_{2}\right)=0.1449$, & $p_{4}>p_{2}>p_{3}>p_{1}$ \\
(Based on the TIT2-WAA operator) & $R\left(A_{3}\right)=0.1369, R\left(A_{4}\right)=0.2253$. & $p_{4}>p_{3}>p_{2}>p_{1}$ \\
Gong et al's approach [13] & $R\left(A_{1}\right)=0.1333, R\left(A_{2}\right)=0.2570$, & $p_{4}>p_{3}>p_{2}>p_{1}$ \\
(Based on the TIT2FEWG operator) & $R\left(A_{3}\right)=0.2707, R\left(A_{4}\right)=0.3390$. & $R\left(A_{1}\right)=0.1447, R\left(A_{2}\right)=0.2464$, \\
Gong et al's approach [14] & $R\left(A_{3}\right)=0.2724, R\left(A_{4}\right)=0.3365$. & $p_{4}>p_{3}>p_{2}>p_{1}$ \\
(Based on the TIT2FWGBM operator) & $R\left(A_{1}\right)=0.5460, R\left(A_{2}\right)=1.5969$, & $R\left(A_{3}\right)=1.3971, R\left(A_{4}\right)=0.9023$. \\
(Based on the Frank operations) & &
\end{tabular}

From Table 5, we find that the created MADM approach, Gong et al's approach [13], and Gong et al's approach [14] derive the same preferred ordering of commercial banks, i.e., $p_{4}>p_{3}>p_{2}>p_{1}$, , which is slightly different to the preferred ordering derived using $\mathrm{Hu}$ et al's approach [18], i.e., $p_{4}>p_{2}>p_{3}>p_{1}$, although the optimal commercial bank is the same for all four approaches. One reason for these methods to derive different preferred orderings of commercial banks is that the created MADM approach, and the existing MADM approaches [13] [14] are all based on the geometric operator, while Hu et al's approach [18] is based on the averaging operator. Moreover, using the geometric operator instead of the averaging operator in Hu et al's approach [18] results in the same preferred ordering of the commercial banks, i.e., $p_{4}>p_{3}>p_{2}>p_{1}$, and all four approaches would derive the same outcome.

In the above section, we have demonstrated the effectiveness of the created approach. However, as the existing approaches [13] [14] and the created approach derive the same preferred ordering of commercial banks, it is hard to illustrate the merits of the created approach well. Thus, in what follows, an application case is provided to conduct a further comparative analysis with the existing approaches [13] [14] [18] that shows the superiority of the created approach.

Example 4. In the context of real MADM problems, the interactions of the attributes are common, ranging from complementariness to redundancy. In addition, because of the reasons of time pressure, lack of knowledge and experts' limited expertise about decision-making problems, the information about attributes may not always be completely known. Considering such situations, Example 3 is modified to presume that the attributes are dependent on the following incomplete attributes' weights information:

$$
\begin{aligned}
& 0.1 \leq \mu\left(\left\{z_{1}\right\}\right) \leq 0.2,0.05 \leq \mu\left(\left\{z_{2}\right\}\right) \leq 0.1,0.1 \leq \mu\left(\left\{z_{3}\right\}\right) \leq 0.2,0.2 \leq \mu\left(\left\{z_{4}\right\}\right) \leq 0.3,0.05 \leq \mu\left(\left\{z_{5}\right\}\right) \leq 0.1, \\
& 0.1 \leq \mu\left(\left\{z_{6}\right\}\right) \leq 0.15,0.05 \leq \mu\left(\left\{z_{7}\right\}\right) \leq 0.1,0.25 \leq \mu\left(\left\{z_{8}\right\}\right) \leq 0.4, \mu\left(\left\{z_{2}, z_{6}\right\}\right) \leq 0.35, \mu\left(\left\{z_{3}, z_{7}\right\}\right) \geq 0.3 .
\end{aligned}
$$

The assessment values remain identical to the Table 3 decision matrix. To illustrate the superiority of the created approach, we use Hu et al's approach [18], Gong et al's approach [13], and Gong et al's approach [14] to handle Example 4. It is noteworthy that both Hu et al's approach [18], and Gong et al's approach [13] assume that the attributes' weights are known, so they cannot directly handle MADM problems with incomplete weights. To apply these two approaches to handle Example 4, we first determine the attributes' weights using the maximum deviation approach [14], which results in the 
same values provided in Table 5. The experimental results from the existing approaches [13] [14] [18] and our created approach are listed in Table 6, where we assume $\gamma=2$ for the created approach.

Table 6. Ranking results from different approaches for Example 4

\begin{tabular}{|c|c|c|}
\hline Approaches & Ranking values (R) & Preferred order \\
\hline $\begin{array}{l}\text { Hu et al's approach [18] } \\
\text { (Based on the TIT2-WAA operator) }\end{array}$ & $\begin{array}{l}R\left(A_{1}\right)=-0.0071, R\left(A_{2}\right)=0.1449 \\
R\left(A_{3}\right)=0.1369, R\left(A_{4}\right)=0.2253\end{array}$ & $p_{4}>p_{2}>p_{3}>p_{1}$ \\
\hline $\begin{array}{l}\text { Gong et al's approach [13] } \\
\text { (Based on the TIT2FEWG operator) }\end{array}$ & $\begin{array}{l}R\left(A_{1}\right)=0.1333, R\left(A_{2}\right)=0.2570 \\
R\left(A_{3}\right)=0.2707, R\left(A_{4}\right)=0.3390\end{array}$ & $p_{4}>p_{3}>p_{2}>p_{1}$ \\
\hline $\begin{array}{l}\text { Gong et al's approach [14] } \\
\text { (Based on the TIT2FWGBM operator) }\end{array}$ & $\begin{array}{l}R\left(A_{1}\right)=0.1447, R\left(A_{2}\right)=0.2464 \\
R\left(A_{3}\right)=0.2724, R\left(A_{4}\right)=0.3365\end{array}$ & $p_{4}>p_{3}>p_{2}>p_{1}$ \\
\hline $\begin{array}{l}\text { The created approach } \\
\text { (Based on the Algebraic operations) }\end{array}$ & $\begin{array}{l}R\left(A_{1}\right)=0.5414, R\left(A_{2}\right)=1.5733 \\
R\left(A_{3}\right)=1.3844, R\left(A_{4}\right)=0.8655\end{array}$ & $p_{4}>p_{3}>p_{2}>p_{1}$ \\
\hline $\begin{array}{l}\text { The created approach } \\
\text { (Based on the Einstein operations) }\end{array}$ & $\begin{array}{l}R\left(A_{1}\right)=0.5507, R\left(A_{2}\right)=1.6279 \\
R\left(A_{3}\right)=1.4126, R\left(A_{4}\right)=0.9503\end{array}$ & $p_{4}>p_{3}>p_{2}>p_{1}$ \\
\hline $\begin{array}{l}\text { The created approach } \\
\text { (Based on the Frank operations) }\end{array}$ & $\begin{array}{l}R\left(A_{1}\right)=0.5460, R\left(A_{2}\right)=1.5969 \\
R\left(A_{3}\right)=1.3971, R\left(A_{4}\right)=0.9023\end{array}$ & $p_{4}>p_{3}>p_{2}>p_{1}$ \\
\hline $\begin{array}{l}\text { The created approach } \\
\text { (Based on the Aczel-Alsina operations) }\end{array}$ & $\begin{array}{l}R\left(A_{1}\right)=0.5063, R\left(A_{2}\right)=1.1830 \\
R\left(A_{3}\right)=1.1600, R\left(A_{4}\right)=0.6240\end{array}$ & $p_{4}>p_{3}>p_{2}>p_{1}$ \\
\hline
\end{tabular}

From Table 6, we observe that when the MADM problem with independent attributes are changed to dependent attributes, Hu et al's approach [18], and Gong et al's approach [13] still result in the same rankings; thus, they cannot handle dependent attributes in Example 4. Here, consultation service and service attitude are negatively related. In general, a commercial bank with a good consultation service also has a satisfied service attitude; thus, the comprehensive weight of the two attributes considered together should be smaller than the sum of the weight of the two attributes when considered alone. Therefore, the ranking results derived by the approaches [13] [18] are not fit for this purpose because they ignore the interactions of the attributes. In addition, it is noteworthy that, in the MADM problem with independent attributes, when they are changed to dependent attributes, Gong et al's approach [14] also keeps the same preferred ordering. Although Gong et al's approach [14] can reflect the homogeneous relationship by aggregated elements, it cannot model the related relationship by the attributes' weights; i.e., it is based on the following equation: $\mu(B)=\sum_{z_{j} \in B} \mu\left(\left\{z_{j}\right\}\right)(\forall B \subset Z)$, which is invalid in Example 4. Therefore, the ranking result derived by Gong et al's approach [14] is also not fit for this purpose. Furthermore, when the MADM problem with independent attributes are changed to dependent attributes, the preferred ordering derived by the created approach changes from $p_{4}>p_{3}>p_{2}>p_{1}$ to $p_{2}>p_{3}>p_{4}>p_{1}$, so the optimal commercial bank changes from $p_{4}$ to $p_{2}$. Obviously, the ranking result of the banks derived by the created approach is reasonable because it is based on the following inequalities:

$$
\begin{aligned}
& \mu\left(\left\{z_{2}, z_{3}\right\}\right)=0.2667<\mu\left(\left\{z_{2}\right\}\right)+\mu\left(\left\{z_{3}\right\}\right)=0.3 ; \mu\left(\left\{z_{2}, z_{6}\right\}\right)=0.35>\mu\left(\left\{z_{2}\right\}\right)+\mu\left(\left\{z_{6}\right\}\right)=0.25 ; \\
& u\left(\left\{z_{3}, z_{7}\right\}\right)=u\left(\left\{z_{3}\right\}\right)+u\left(\left\{z_{7}\right\}\right)=0.3 .
\end{aligned}
$$

Thus, the created approach can capture the negative relationship of attributes $z_{2}$ and $z_{3}$, the positive relationship of the attributes $z_{2}$ and $z_{6}$, and the independent relationship between the attributes $z_{3}$ and $z_{7}$. 
Therefore, the created approach derives a realistic ranking result, which is not the case with the existing approaches [13] [14] [18].

\section{Conclusions}

This article focused on MADM in an IT2FNs context for service quality evaluation of commercial banks based on the ATs, the Choquet integral and the generalized Banzhaf index. First, novel operations of IT2FNs based on the ATs were defined and shown to be more versatile and flexible when fusing fuzzy information rather than the existing operations of IT2FNs. Then, the T2FAC operator was proposed, and it not only weights the aggregated arguments or their ordered positions but also considers the related characteristics of the aggregated arguments or their ordered positions. However, the T2FAC operator results in information loss because not all coalitions of attributes are considered. For this reason, the BT2FAC operator was proposed to globally capture the interactions of the attributes. Because the BT2FAC operator is based on the FM, and the FM is defined on the power set, it makes the problem complexity exponential. To enhance the practicality of the BT2FAC operator, the 2ABT2FAC operator was proposed. Besides, when the weighting information is partly known, two linear programming models for the optimal FMs were constructed based on the developed bi-directional projection measure of IT2FNs and the Banzhaf function. As a series of development, two methods were created for handling IT2F MADM problems with incomplete weight information and independent attributes. Moreover, we provided a case concerning the service quality evaluation of commercial banks to illustrate the specific application and the superiority of the created approach. From the experimental results shown in Example 3 and Example 4, we could see that the proposed method could overcome the drawbacks of Hu et al.'s approach [18], Gong et al.'s approach [13] and Gong et al.'s approach [14] for MADM under IT2F environments. The proposed approaches offer us a helpful way for IT2F MADM. The major contributions of this article are: 1) the defined new operations of IT2FNs can better aggregate input arguments and ensure the accuracy of final results; 2) the proposed IT2F Choquet operators can globally reflect the heterogeneous relationship of the criteria; 3 ) the constructed models can objectively determine attributes' weights and avoid subjective randomness; 4) the created MADM approaches can effectively handle service quality evaluation problems. As future work, we want to explore the created MADM methods in the field of different areas, such as mobile phone evaluation [30], group recommender systems [3] and investment management [29]. Besides, we shall propose some MADM methods based on intelligent optimization algorithms [49] and granular computing techniques [11] [12].

\section{Acknowledgements}

This article was funded by the National Natural Science Foundation of China (Nos. 71771140, 71471172), 文化名家暨 “四个一批” 人才项目(Project of cultural masters and “the four kinds of a batch" talents), the Special Funds of Taishan Scholars Project of Shandong Province (No. ts201511045).

\section{References}

[1] F. Akiyoshi, Effects of separating commercial and investment banking: Evidence from the 
dissolution of a joint venture investment bank, Journal of Financial Economics, 2019, https://doi.org/10.1016/j.jfineco.2019.05.004.

[2] M. Bottero, V. Ferretti, J.R. Figueira, S. Greco, B. Roy, On the Choquet multiple criteria preference aggregation model: Theoretical and practical insights from a real-world application, European Journal of Operational Research 271 (1) (2018) 120-140.

[3] N. Capuano, F. Chiclana, E. Herrera-Viedma, H. Fujita, V. Loia, Fuzzy Group Decision Making for influence-aware recommendations, Computers in Human Behavior 101 (2019) 371-379.

[4] S.M. Chen, J.A. Hong, Fuzzy Multiple Attributes Group Decision-Making Based on Ranking Interval Type-2 Fuzzy Sets and the TOPSIS Method, IEEE Transactions on Systems, Man, and Cybernetics: Systems 44 (12) (2014) 1665-1673.

[5] S.M. Chen, L.W. Lee, Fuzzy multiple attributes group decision-making based on the ranking values and the arithmetic operations of interval type-2 fuzzy sets, Expert Systems with Applications 37 (1) (2010) 824-833.

[6] S. Das, D. Guha, R. Mesiar, Extended Bonferroni Mean Under Intuitionistic Fuzzy Environment Based on a Strict t-Conorm, IEEE Transactions on Systems, Man, and Cybernetics: Systems 47 (8) (2017) 2083-2099.

[7] Y.Z. Dong, M. Firth, W.X. Hou, W.W. Yang, Evaluating the performance of Chinese commercial banks: A comparative analysis of different types of banks, European Journal of Operational Research 252 (1) (2016) 280-295.

[8] E. Duncan, G. Elliot, Customer Service Quality and Financial Performance among Australian Retail Financial Institutions, Journal of Financial Services Marketing 7 (2002) 25-41.

[9] B. Dutta, D. Guha, R. Mesiar, A Model Based on Linguistic 2-Tuples for Dealing with Heterogeneous Relationship among Attributes in Multi-expert Decision Making, IEEE Transactions on Fuzzy Systems 23(5) (2015) 1817-1831.

[10] H. Fujita, A. Gaeta, V. Loia, F. Orciuoli, Hypotheses Analysis and Assessment in counter-terrorism activities: a method based on OWA and Fuzzy Probabilistic Rough Sets, IEEE Transactions on Fuzzy Systems (2019) 1-15, doi: 10.1109/TFUZZ.2019.2955047.

[11] H. Fujita, A. Gaeta, V. Loia, F. Orciuoli, Improving awareness in early stages of security analysis: A zone partition method based on GrC, Applied Intelligence 49 (3) (2019) 1063-1077.

[12] H. Fujita, A. Gaeta, V. Loia, F. Orciuoli, Resilience Analysis of Critical Infrastructures: A Cognitive Approach Based on Granular Computing, IEEE Transactions on Cybernetics 49 (5) (2019) 1835-1848.

[13] Y.B. Gong, L.L. Dai, N. Hu, Interval type-2 fuzzy information aggregation based on Einstein operations and its application to decision making, International Journal of Innovative Computing, Information and Control 12 (6) (2016) 2011-2026.

[14] Y.B. Gong, N. Hu, J.G. Zhang, G.F. Liu, J.G. Deng, Multi-attribute group decision making method based on geometric Bonferroni mean operator of trapezoidal interval type-2 fuzzy numbers, Computers \& Industrial Engineering 81 (2015) 167-176.

[15] M. Grabisch, Fuzzy integral in multicriteria decision making, Fuzzy sets and Systems 69 (3) (1995) 279-298. 
[16] M. Grabisch, K-order additive discrete fuzzy measures and their representation, Fuzzy Sets and Systems 92 (2) (1997) 167-189.

[17] P. Hajek, W. Froelich, Integrating TOPSIS with interval-valued intuitionistic fuzzy cognitive maps for effective group decision making, Information Sciences 485 (2019) 394-412.

[18] J.H. Hu, Y. Zhang, X.H. Chen, Y.M. Liu, Multi-criteria decision making method based on possibility degree of interval type-2 fuzzy number, Knowledge-Based Systems 43 (2013) 21-29.

[19] Y.B. Ju, A.H. Wang, Projection method for multiple criteria group decision making with incomplete weight information in linguistic setting, Applied Mathematical Modelling 37 (20-21) (2013) 9031-9040.

[20] O.M. Karatepe, U. Yavas, E. Babakus, Measuring service quality of banks: Scale development and validation, Journal of Retailing \& Consumer Services 12 (2005) 373-383.

[21] C. Liu, G.L. Tang, P.D. Liu, C.Q. Liu, Hesitant Fuzzy Linguistic Archimedean Aggregation Operators in Decision Making with the Dempster-Shafer Belief Structure, International Journal of Fuzzy Systems 21(5) (2019) 1330-1348.

[22] P.D. Liu, S.M. Chen, G.L. Tang, Multicriteria Decision Making With Incomplete Weights Based on 2-D Uncertain Linguistic Choquet Integral Operators, IEEE Transactions on Cybernetics (2019) 1-15, doi: 10.1109/TCYB.2019. 2913639.

[23] P.D. Liu, S.M. Chen, P. Wang, Multiple-attribute group decision-making based on q-rung orthopair fuzzy power maclaurin symmetric mean operators, IEEE Transactions on Systems, Man and Cybernetics: Systems (2018) 1-16, doi: 10.1109/TSMC. 2018.2852948.

[24] P.D. Liu, G.L. Tang, Multi-criteria group decision-making based on interval neutrosophic uncertain linguistic variables and Choquet integral, Cognitive Computation 8 (6) (2016) 1036-1056

[25] P.D. Liu, G.L. Tang, Some power generalized aggregation operators based on the interval neutrosophic sets and their application to decision making, Journal of Intelligent \& Fuzzy Systems 30 (5) (2016) 2517-2528.

[26] X.Y. Ma, P. Wu, L.G. Zhou, H.Y. Chen, T. Zheng, J.Q. Ge, Approaches Based on Interval Type-2 Fuzzy Aggregation Operators for Multiple Attribute Group Decision Making, International Journal of Fuzzy Systems 18 (4) (2016) 697-715.

[27] J.L. Marichal, The influence of variables on pseudo-Boolean functions with applications to game theory and multicriteria decision making, Discrete Applied Mathematics 107 (1-3) (2000) 139-164.

[28] J.M. Mendel, R.I.B. John, Type-2 fuzzy sets made simple, IEEE Transactions on Fuzzy Systems 10 (2) (2002) 117-127.

[29] F.Y. Meng, J. Tang, H. Fujita, Consistency-Based Algorithms for Decision-Making With Interval Fuzzy Preference Relations, IEEE Transactions on Fuzzy Systems 27 (10) (2019) 2052 - 2066.

[30] F.Y. Meng, J. Tang, H. Fujita, Linguistic intuitionistic fuzzy preference relations and their application to multi-criteria decision making, Information Fusion 46 (2019) 77 - 90.

[31] F.Y. Meng, Q. Zhang, J.Q. Zhan, The interval-valued intuitionistic fuzzy geometric Choquet aggregation operator based on the generalized Banzhaf index and 2-additive measure, 
Technological \& Economic Development 21 (2) (2015) 86-215.

[32] J.D. Qin, Interval type-2 fuzzy Hamy mean operators and their application in multiple criteria decision making, Granular Computing 2 (4) (2017) 249-269.

[33] J.D. Qin, X.W. Liu, Frank Aggregation Operators for Triangular Interval Type-2 Fuzzy Set and Its Application in Multiple Attribute Group Decision Making, Journal of Applied Mathematics 2014 (2014) 1-24.

[34] J.Q. Qin, X.W. Liu, Multi-attribute group decision making using combined ranking value under interval type-2 fuzzy environment, Information Sciences 297 (2015) 293-315.

[35] J.D. Qin, X.W. Liu, W. Pedrycz, An extended TODIM multi-criteria group decision making method for green supplier selection in interval type-2 fuzzy environment, European Journal of Operational Research 258 (2) (2017) 626-638.

[36] F.H. Shu, Evaluation of Service Quality of Commercial Banks Based on Prospect Theory, Journal of Shanghai Lixin University of Accounting and Finance 2 (2018) 76-85.

[37] M. Sugeno, Theory of fuzzy integral and its application, Ph.D. Dissertation, Tokyo Institute of Technology, Tokyo, Japan, 1974.

[38] L. Sun, H. Dong, A.X. Liu, Aggregation Functions Considering Criteria Interrelationships in Fuzzy Multi-Criteria Decision Making: State-of-the-Art, IEEE Access 6 (2018) 68104-68136.

[39] C. Tian, J.J. Peng, S. Zhang, W.Y. Zhang, J.Q. Wang, Weighted picture fuzzy aggregation operators and their applications to multi-criteria decision-making problems, Computers \& Industrial Engineering 137 (2019), https://doi.org/10.1016/j.cie.2019.106037.

[40] Z.P. Tian, R.X. Nie, J.Q. Wang, Social network analysis-based consensus-supporting framework for large-scale group decision-making with incomplete interval type-2 fuzzy information, Information Sciences 502 (2019) 446-471.

[41] R. Ureña, G. Kou, J. Wu, F. Chiclana, E. Herrera-Viedma, Dealing with Incomplete Information in Linguistic Group Decision Making by Means of Interval Type 2 Fuzzy Sets, International Journal of Intelligent Systems 34 (6) (2019) 1261-1280.

[42] H.H. Wang, P.D. Liu, Z.M. Liu, Trapezoidal interval type-2 fuzzy Maclaurin symmetric mean operators and their applications to multiple attribute group decision making, International Journal for Uncertainty Quantification 8 (4) (2018) 343-360.

[43] H.H. Wang, Y.B. Ju, P.D. Liu, D.W. Ju, Z.M. Liu, Some trapezoidal interval type-2 fuzzy Heronian mean operators and their application in multiple attribute group decision making, Journal of Intelligent and Fuzzy Systems 35 (2018) 2323-2337.

[44] L. Wang, H.Y. Zhang, J.Q. Wang, L. Li, Picture fuzzy normalized projection-based VIKOR method for the risk evaluation of construction project, Applied Soft Computing 64 (2018) 216-226.

[45] X.T. Wang, E. Triantaphyllou, Ranking irregularities when evaluating alternatives by using some ELECTRE methods, Omega 36 (2008) 45-63.

[46] V. Yilmaz, E. Ari, H. Gurbuz, Investigating the relationship between service quality dimensions, customer satisfaction and loyalty in Turkish banking sector, International Journal of bank marketing 36 (3) (2018) 423-440.

[47] L.A. Zadeh, The concept of a linguistic variable and its application to approximate reasoning-I, Information sciences 8 (3) (1975) 199-249. 
[48] P. Zhang, Q.S. Gao, Analysis of Affecting Factors for Commercial Banks' Service Quality Based on QFD, Journal of Qingdao University 26 (1) (2013) 80-83.

[49] X.J. Zhou, C.H. Yang, W.H. Gui, A Statistical Study on Parameter Selection of Operators in Continuous State Transition Algorithm, IEEE Transactions on Cybernetics 49 (10) (2019) 37223730 .

[50] N. Zhu, J.L. Hougaard, Z.Q. Yu, B. Wang, Ranking Chinese commercial banks based on their expected impact on structural efficiency, Omega, 2019, https://doi.org/10.1016/j.omega.2019. $\underline{03.007 .}$ 


\section{APPENDIX 1.}

Theorem 1. The aggregation outputs of the set $\Lambda=\left\{A_{1}, A_{2}, \ldots, A_{n}\right\}$ of trapezoidal IT2FNs by the IT2FAC operator is the trapezoidal IT2FN:

$$
\begin{aligned}
& \operatorname{IT} 2 F A C\left(A_{1}, A_{2}, \ldots, A_{n}\right) \\
&=\left(\left(g^{-1}\left(\sum_{k=1}^{n}\left(\mu\left(Z_{(k)}\right)-\mu\left(Z_{(k+1)}\right)\right) g\left(a_{(k) 1}^{u}\right)\right), g^{-1}\left(\sum_{k=1}^{n}\left(\mu\left(Z_{(k)}\right)-\mu\left(Z_{(k+1)}\right)\right) g\left(a_{(k) 2}^{u}\right)\right),\right.\right. \\
& g^{-1}\left(\sum_{k=1}^{n}\left(\mu\left(Z_{(k)}\right)-\mu\left(Z_{(k+1)}\right)\right) g\left(a_{(k) 3}^{u}\right)\right), g^{-1}\left(\sum_{k=1}^{n}\left(\mu\left(Z_{(k)}\right)-\mu\left(Z_{(k+1)}\right)\right) g\left(a_{(k) 4}^{u}\right)\right) ; \\
&\left.g^{-1}\left(\sum_{k=1}^{n}\left(\mu\left(Z_{(k)}\right)-\mu\left(Z_{(k+1)}\right)\right) g\left(h_{1}\left(A_{(k)}^{u}\right)\right)\right), g^{-1}\left(\sum_{k=1}^{n}\left(\mu\left(Z_{(k)}\right)-\mu\left(Z_{(k+1)}\right)\right) g\left(h_{2}\left(A_{(k)}^{u}\right)\right)\right)\right), \\
& \quad\left(g^{-1}\left(\sum_{k=1}^{n}\left(\mu\left(Z_{(k)}\right)-\mu\left(Z_{(k+1)}\right)\right) g\left(a_{(k) 1}^{l}\right)\right), g^{-1}\left(\sum_{k=1}^{n}\left(\mu\left(Z_{(k)}\right)-\mu\left(Z_{(k+1)}\right)\right) g\left(a_{(k) 2}^{l}\right)\right),\right. \\
& g^{-1}\left(\sum_{k=1}^{n}\left(\mu\left(Z_{(k)}\right)-\mu\left(Z_{(k+1)}\right)\right) g\left(a_{(k) 3}^{l}\right)\right), g^{-1}\left(\sum_{k=1}^{n}\left(\mu\left(Z_{(k)}\right)-\mu\left(Z_{(k+1)}\right)\right) g\left(a_{(k) 4}^{l}\right)\right) ; \\
&\left.\left.\left.\left(\mu\left(Z_{(k)}\right)-\mu\left(Z_{(k+1)}\right)\right) g\left(h_{1}\left(A_{(k)}^{l}\right)\right)\right), g^{-1}\left(\sum_{k=1}^{n}\left(\mu\left(Z_{(k)}\right)-\mu\left(Z_{(k+1)}\right)\right) g\left(h_{2}\left(A_{(k)}^{l}\right)\right)\right)\right)\right) .
\end{aligned}
$$

Proof. We prove that Eq. (17) holds and the result of Eq. (17) is a trapezoidal IT2FN.

(1) We first prove that Eq. (17) holds by mathematical induction on $n$.

a) For $n=2$, since $0 \leq \mu\left(Z_{(k)}\right)-\mu\left(Z_{(k+1)}\right) \leq 1$ for any permutation (.) of the elements in the set $Z$, by the operations of trapezoidal IT2FNs, the following is derived:

$$
\begin{gathered}
I T 2 F A C\left(A_{1}, A_{2}\right)=\otimes_{k=1}^{2} A_{(k)}{ }^{\mu\left(Z_{(k)}\right)-\mu\left(Z_{(k+1)}\right)}=A_{(1)}{ }^{\mu\left(Z_{(1)}\right)-\mu\left(Z_{(2)}\right)} \otimes A_{(2)}{ }^{\mu\left(Z_{(2)}\right)-\mu\left(Z_{(3)}\right)} \\
=\left(\left(g^{-1}\left(\left(\mu\left(Z_{(1)}\right)-\mu\left(Z_{(2)}\right)\right) g\left(a_{(1) 1}^{u}\right)\right), g^{-1}\left(\left(\mu\left(Z_{(1)}\right)-\mu\left(Z_{(2)}\right)\right) g\left(a_{(1) 2}^{u}\right)\right),\right.\right. \\
g^{-1}\left(\left(\mu\left(Z_{(1)}\right)-\mu\left(Z_{(2)}\right)\right) g\left(a_{(1) 3}^{u}\right)\right), g^{-1}\left(\left(\mu\left(Z_{(1)}\right)-\mu\left(Z_{(2)}\right)\right) g\left(a_{(1) 4}^{u}\right)\right) ; \\
\left.g^{-1}\left(\left(\mu\left(Z_{(1)}\right)-\mu\left(Z_{(2)}\right)\right) g\left(h_{1}\left(A_{(1)}^{u}\right)\right)\right), g^{-1}\left(\left(\mu\left(Z_{(1)}\right)-\mu\left(Z_{(2)}\right)\right) g\left(h_{2}\left(A_{(1)}^{u}\right)\right)\right)\right), \\
\left(g^{-1}\left(\left(\mu\left(Z_{(1)}\right)-\mu\left(Z_{(2)}\right)\right) g\left(a_{(1) 1}^{l}\right)\right), g^{-1}\left(\left(\mu\left(Z_{(1)}\right)-\mu\left(Z_{(2)}\right)\right) g\left(a_{(1) 2}^{l}\right)\right),\right. \\
g^{-1}\left(\left(\mu\left(Z_{(1)}\right)-\mu\left(Z_{(2)}\right)\right) g\left(a_{(1) 3}^{l}\right)\right), g^{-1}\left(\left(\mu\left(Z_{(1)}\right)-\mu\left(Z_{(2)}\right)\right) g\left(a_{(1) 4}^{l}\right)\right) ; \\
\left.\left.g^{-1}\left(\left(\mu\left(Z_{(1)}\right)-\mu\left(Z_{(2)}\right)\right) g\left(h_{1}\left(A_{(1)}^{l}\right)\right)\right), g^{-1}\left(\left(\mu\left(Z_{(1)}\right)-\mu\left(Z_{(2)}\right)\right) g\left(h_{2}\left(A_{(1)}^{l}\right)\right)\right)\right)\right) \\
\otimes\left(\left(g^{-1}\left(\left(\mu\left(Z_{(2)}\right)-\mu\left(Z_{(3)}\right)\right) g\left(a_{(2) 1}^{u}\right)\right), g^{-1}\left(\left(\mu\left(Z_{(2)}\right)-\mu\left(Z_{(3)}\right)\right) g\left(a_{(2) 2}^{u}\right)\right),\right.\right. \\
g^{-1}\left(\left(\mu\left(Z_{(2)}\right)-\mu\left(Z_{(3)}\right)\right) g\left(a_{(2) 3}^{u}\right)\right), g^{-1}\left(\left(\mu\left(Z_{(2)}\right)-\mu\left(Z_{(3)}\right)\right) g\left(a_{(2) 4}^{u}\right)\right) ; \\
g^{-1}\left(\left(\mu\left(Z_{(2)}\right)-\mu\left(Z_{(3)}\right)\right) g\left(h_{1}\left(A_{(2)}^{u}\right)\right)\right), g^{-1}\left(\left(\mu\left(Z_{(2)}\right)-\mu\left(Z_{(3)}\right)\right) g\left(h_{2}\left(A_{(2)}^{u}\right)\right)\right), \\
\left(g^{-1}\left(\left(\mu\left(Z_{(2)}\right)-\mu\left(Z_{(3)}\right)\right) g\left(a_{(2) 1}^{l}\right)\right), g^{-1}\left(\left(\mu\left(Z_{(2)}\right)-\mu\left(Z_{(3)}\right)\right) g\left(a_{(2) 2}^{l}\right)\right),\right. \\
g^{-1}\left(\left(\mu\left(Z_{(2)}\right)-\mu\left(Z_{(3)}\right)\right) g\left(a_{(2) 3}^{l}\right)\right), g^{-1}\left(\left(\mu\left(Z_{(2)}\right)-\mu\left(Z_{(3)}\right)\right) g\left(a_{(2) 4}^{l}\right)\right) ; \\
\left.\left.g^{-1}\left(\left(\mu\left(Z_{(2)}\right)-\mu\left(Z_{(3)}\right)\right) g\left(h_{1}\left(A_{(2)}^{l}\right)\right)\right), g^{-1}\left(\left(\mu\left(Z_{(2)}\right)-\mu\left(Z_{(3)}\right)\right) g\left(h_{2}\left(A_{(2)}^{l}\right)\right)\right)\right)\right)
\end{gathered}
$$




$$
\begin{array}{r}
=\left(\left(g^{-1}\left(\sum_{k=1}^{2}\left(\mu\left(Z_{(k)}\right)-\mu\left(Z_{(k+1)}\right)\right) g\left(a_{(k) 1}^{u}\right)\right), g^{-1}\left(\sum_{k=1}^{2}\left(\mu\left(Z_{(k)}\right)-\mu\left(Z_{(k+1)}\right)\right) g\left(a_{(k) 2}^{u}\right)\right),\right.\right. \\
g^{-1}\left(\sum_{k=1}^{2}\left(\mu\left(Z_{(k)}\right)-\mu\left(Z_{(k+1)}\right)\right) g\left(a_{(k) 3}^{u}\right)\right), g^{-1}\left(\sum_{k=1}^{2}\left(\mu\left(Z_{(k)}\right)-\mu\left(Z_{(k+1)}\right)\right) g\left(a_{(k) 4}^{u}\right)\right) ; \\
\left.g^{-1}\left(\sum_{k=1}^{2}\left(\mu\left(Z_{(k)}\right)-\mu\left(Z_{(k+1)}\right)\right) g\left(h_{1}\left(A_{(k)}^{u}\right)\right)\right), g^{-1}\left(\sum_{k=1}^{2}\left(\mu\left(Z_{(k)}\right)-\mu\left(Z_{(k+1)}\right)\right) g\left(h_{2}\left(A_{(k)}^{u}\right)\right)\right)\right), \\
\quad\left(g^{-1}\left(\sum_{k=1}^{2}\left(\mu\left(Z_{(k)}\right)-\mu\left(Z_{(k+1)}\right)\right) g\left(a_{(k) 1}^{l}\right)\right), g^{-1}\left(\sum_{k=1}^{2}\left(\mu\left(Z_{(k)}\right)-\mu\left(Z_{(k+1)}\right)\right) g\left(a_{(k) 2}^{l}\right)\right),\right. \\
g^{-1}\left(\sum_{k=1}^{2}\left(\mu\left(Z_{(k)}\right)-\mu\left(Z_{(k+1)}\right)\right) g\left(a_{(k) 3}^{l}\right)\right), g^{-1}\left(\sum_{k=1}^{2}\left(\mu\left(Z_{(k)}\right)-\mu\left(Z_{(k+1)}\right)\right) g\left(a_{(k) 4}^{l}\right)\right) ; \\
\left.\left.g^{-1}\left(\sum_{k=1}^{2}\left(\mu\left(Z_{(k)}\right)-\mu\left(Z_{(k+1)}\right)\right) g\left(h_{1}\left(A_{(k)}^{l}\right)\right)\right), g^{-1}\left(\sum_{k=1}^{2}\left(\mu\left(Z_{(k)}\right)-\mu\left(Z_{(k+1)}\right)\right) g\left(h_{2}\left(A_{(k)}^{l}\right)\right)\right)\right)\right) .
\end{array}
$$

b) It is assumed that Eq. (17) holds for $n=m$, i.e.:

$$
\operatorname{IT} 2 F A C\left(A_{1}, A_{2}, \ldots, A_{m}\right)
$$

$$
\begin{array}{r}
=\left(\left(g^{-1}\left(\sum_{k=1}^{m}\left(\mu\left(Z_{(k)}\right)-\mu\left(Z_{(k+1)}\right)\right) g\left(a_{(k) 1}^{u}\right)\right), g^{-1}\left(\sum_{k=1}^{m}\left(\mu\left(Z_{(k)}\right)-\mu\left(Z_{(k+1)}\right)\right) g\left(a_{(k) 2}^{u}\right)\right),\right.\right. \\
g^{-1}\left(\sum_{k=1}^{m}\left(\mu\left(Z_{(k)}\right)-\mu\left(Z_{(k+1)}\right)\right) g\left(a_{(k) 3}^{u}\right)\right), g^{-1}\left(\sum_{k=1}^{m}\left(\mu\left(Z_{(k)}\right)-\mu\left(Z_{(k+1)}\right)\right) g\left(a_{(k) 4}^{u}\right)\right) \\
\left.g^{-1}\left(\sum_{k=1}^{m}\left(\mu\left(Z_{(k)}\right)-\mu\left(Z_{(k+1)}\right)\right) g\left(h_{1}\left(A_{(k)}^{u}\right)\right)\right), g^{-1}\left(\sum_{k=1}^{m}\left(\mu\left(Z_{(k)}\right)-\mu\left(Z_{(k+1)}\right)\right) g\left(h_{2}\left(A_{(k)}^{u}\right)\right)\right)\right) \\
\left(g^{-1}\left(\sum_{k=1}^{m}\left(\mu\left(Z_{(k)}\right)-\mu\left(Z_{(k+1)}\right)\right) g\left(a_{(k) 1}^{l}\right)\right), g^{-1}\left(\sum_{k=1}^{m}\left(\mu\left(Z_{(k)}\right)-\mu\left(Z_{(k+1)}\right)\right) g\left(a_{(k) 2}^{l}\right)\right),\right. \\
g^{-1}\left(\sum_{k=1}^{m}\left(\mu\left(Z_{(k)}\right)-\mu\left(Z_{(k+1)}\right)\right) g\left(a_{(k) 3}^{l}\right)\right), g^{-1}\left(\sum_{k=1}^{m}\left(\mu\left(Z_{(k)}\right)-\mu\left(Z_{(k+1)}\right)\right) g\left(a_{(k) 4}^{l}\right)\right) \\
\left.\left.g^{-1}\left(\sum_{k=1}^{m}\left(\mu\left(Z_{(k)}\right)-\mu\left(Z_{(k+1)}\right)\right) g\left(h_{1}\left(A_{(k)}^{l}\right)\right)\right), g^{-1}\left(\sum_{k=1}^{m}\left(\mu\left(Z_{(k)}\right)-\mu\left(Z_{(k+1)}\right)\right) g\left(h_{2}\left(A_{(k)}^{l}\right)\right)\right)\right)\right) .
\end{array}
$$

c) For $n=m+1$, the following is derived:

$$
\begin{aligned}
& \operatorname{IT2FAC}\left(A_{1}, A_{2}, \ldots, A_{m+1}\right)=\bigotimes_{k=1}^{m+1} A_{(k)}{ }^{\mu\left(Z_{(k)}\right)-\mu\left(Z_{(k+1)}\right)}=\bigotimes_{k=1}^{m} A_{(k)}{ }^{\mu\left(Z_{(k)}\right)-\mu\left(Z_{(k+1)}\right)} \otimes A_{(m+1)} \mu\left(Z_{(m+1)}\right)-\mu\left(Z_{(m+2)}\right) \\
& =\left(\left(g^{-1}\left(\sum_{k=1}^{m}\left(\mu\left(Z_{(k)}\right)-\mu\left(Z_{(k+1)}\right)\right) g\left(a_{(k) 1}^{u}\right)\right), g^{-1}\left(\sum_{k=1}^{m}\left(\mu\left(Z_{(k)}\right)-\mu\left(Z_{(k+1)}\right)\right) g\left(a_{(k) 2}^{u}\right)\right)\right.\right. \text {, } \\
& g^{-1}\left(\sum_{k=1}^{m}\left(\mu\left(Z_{(k)}\right)-\mu\left(Z_{(k+1)}\right)\right) g\left(a_{(k) 3}^{u}\right)\right), g^{-1}\left(\sum_{k=1}^{m}\left(\mu\left(Z_{(k)}\right)-\mu\left(Z_{(k+1)}\right)\right) g\left(a_{(k) 4}^{u}\right)\right) ; \\
& \left.g^{-1}\left(\sum_{k=1}^{m}\left(\mu\left(Z_{(k)}\right)-\mu\left(Z_{(k+1)}\right)\right) g\left(h_{1}\left(A_{(k)}^{u}\right)\right)\right), g^{-1}\left(\sum_{k=1}^{m}\left(\mu\left(Z_{(k)}\right)-\mu\left(Z_{(k+1)}\right)\right) g\left(h_{2}\left(A_{(k)}^{u}\right)\right)\right)\right), \\
& \left(g^{-1}\left(\sum_{k=1}^{m}\left(\mu\left(Z_{(k)}\right)-\mu\left(Z_{(k+1)}\right)\right) g\left(a_{(k) 1}^{l}\right)\right), g^{-1}\left(\sum_{k=1}^{m}\left(\mu\left(Z_{(k)}\right)-\mu\left(Z_{(k+1)}\right)\right) g\left(a_{(k) 2}^{l}\right)\right),\right. \\
& g^{-1}\left(\sum_{k=1}^{m}\left(\mu\left(Z_{(k)}\right)-\mu\left(Z_{(k+1)}\right)\right) g\left(a_{(k) 3}^{l}\right)\right), g^{-1}\left(\sum_{k=1}^{m}\left(\mu\left(Z_{(k)}\right)-\mu\left(Z_{(k+1)}\right)\right) g\left(a_{(k) 4}^{l}\right)\right) ; \\
& \left.\left.g^{-1}\left(\sum_{k=1}^{m}\left(\mu\left(Z_{(k)}\right)-\mu\left(Z_{(k+1)}\right)\right) g\left(h_{1}\left(A_{(k)}^{l}\right)\right)\right), g^{-1}\left(\sum_{k=1}^{m}\left(\mu\left(Z_{(k)}\right)-\mu\left(Z_{(k+1)}\right)\right) g\left(h_{2}\left(A_{(k)}^{l}\right)\right)\right)\right)\right)
\end{aligned}
$$




$$
\begin{array}{r}
\otimes\left(\left(g^{-1}\left(\left(\mu\left(Z_{(m+1)}\right)-\mu\left(Z_{(m+2)}\right)\right) g\left(a_{(m+1) 1}^{u}\right)\right), g^{-1}\left(\left(\mu\left(Z_{(m+1)}\right)-\mu\left(Z_{(m+2)}\right)\right) g\left(a_{(m+1) 2}^{u}\right)\right),\right.\right. \\
g^{-1}\left(\left(\mu\left(Z_{(m+1)}\right)-\mu\left(Z_{(m+2)}\right)\right) g\left(a_{(m+1) 3}^{u}\right)\right), g^{-1}\left(\left(\mu\left(Z_{(m+1)}\right)-\mu\left(Z_{(m+2)}\right)\right) g\left(a_{(m+1) 4}^{u}\right)\right) ; \\
g^{-1}\left(\left(\mu\left(Z_{(m+1)}\right)-\mu\left(Z_{(m+2)}\right)\right) g\left(h_{1}\left(A_{(m+1)}^{u}\right)\right)\right), g^{-1}\left(\left(\mu\left(Z_{(m+1)}\right)-\mu\left(Z_{(m+2)}\right)\right) g\left(h_{2}\left(A_{(m+1)}^{u}\right)\right)\right), \\
\left(g^{-1}\left(\left(\mu\left(Z_{(m+1)}\right)-\mu\left(Z_{(m+2)}\right)\right) g\left(a_{(m+1) 1}^{l}\right)\right), g^{-1}\left(\left(\mu\left(Z_{(m+1)}\right)-\mu\left(Z_{(m+2)}\right)\right) g\left(a_{(m+1) 2}^{l}\right)\right),\right. \\
g^{-1}\left(\left(\mu\left(Z_{(m+1)}\right)-\mu\left(Z_{(m+2)}\right)\right) g\left(a_{(m+1) 3}^{l}\right)\right), g^{-1}\left(\left(\mu\left(Z_{(m+1)}\right)-\mu\left(Z_{(m+2)}\right)\right) g\left(a_{(m+1) 4}^{l}\right)\right) ; \\
\left.g^{-1}\left(\left(\mu\left(Z_{(m+1)}\right)-\mu\left(Z_{(m+2)}\right)\right) g\left(h_{1}\left(A_{(m+1)}^{l}\right)\right)\right), g^{-1}\left(\left(\mu\left(Z_{(m+1)}\right)-\mu\left(Z_{(m+2)}\right)\right) g\left(h_{2}\left(A_{(m+1)}^{l}\right)\right)\right)\right) \\
=\left(\left(g^{-1}\left(\sum_{k=1}^{m+1}\left(\mu\left(Z_{(k)}\right)-\mu\left(Z_{(k+1)}\right)\right) g\left(a_{(k) 1}^{u}\right)\right), g^{-1}\left(\sum_{k=1}^{m+1}\left(\mu\left(Z_{(k)}\right)-\mu\left(Z_{(k+1)}\right)\right) g\left(a_{(k) 2}^{u}\right)\right),\right.\right. \\
g^{-1}\left(\sum_{k=1}^{m+1}\left(\mu\left(Z_{(k)}\right)-\mu\left(Z_{(k+1)}\right)\right) g\left(a_{(k) 3}^{u}\right)\right), g^{-1}\left(\sum_{k=1}^{m+1}\left(\mu\left(Z_{(k)}\right)-\mu\left(Z_{(k+1)}\right)\right) g\left(a_{(k) 4}^{u}\right)\right) ; \\
\left.g^{-1}\left(\sum_{k=1}^{m+1}\left(\mu\left(Z_{(k)}\right)-\mu\left(Z_{(k+1)}\right)\right) g\left(h_{1}\left(A_{(k)}^{u}\right)\right)\right), g^{-1}\left(\sum_{k=1}^{m+1}\left(\mu\left(Z_{(k)}\right)-\mu\left(Z_{(k+1)}\right)\right) g\left(h_{2}\left(A_{(k)}^{u}\right)\right)\right)\right), \\
\left(g^{-1}\left(\sum_{k=1}^{m+1}\left(\mu\left(Z_{(k)}\right)-\mu\left(Z_{(k+1)}\right)\right) g\left(a_{(k) 1}^{l}\right)\right), g^{-1}\left(\sum_{k=1}^{m+1}\left(\mu\left(Z_{(k)}\right)-\mu\left(Z_{(k+1)}\right)\right) g\left(a_{(k) 2}^{l}\right)\right),\right. \\
g^{-1}\left(\sum_{k=1}^{m+1}\left(\mu\left(Z_{(k)}\right)-\mu\left(Z_{(k+1)}\right)\right) g\left(a_{(k) 3}^{l}\right)\right), g^{-1}\left(\sum_{k=1}^{m+1}\left(\mu\left(Z_{(k)}\right)-\mu\left(Z_{(k+1)}\right)\right) g\left(a_{(k) 4}^{l}\right)\right) ; \\
\left.\left.g^{-1}\left(\sum_{k=1}^{m+1}\left(\mu\left(Z_{(k)}\right)-\mu\left(Z_{(k+1)}\right)\right) g\left(h_{1}\left(A_{(k)}^{l}\right)\right)\right), g^{-1}\left(\sum_{k=1}^{m+1}\left(\mu\left(Z_{(k)}\right)-\mu\left(Z_{(k+1)}\right)\right) g\left(h_{2}\left(A_{(k)}^{l}\right)\right)\right)\right)\right) .
\end{array}
$$

Thus, by a), b) and c), it is proved that Eq. (17) holds.

(2) We prove that Eq. (17) is a trapezoidal IT2FN. It is assumed that:

$$
\begin{aligned}
& b_{j}^{t}=g^{-1}\left(\sum_{k=1}^{n}\left(\mu\left(Z_{(k)}\right)-\mu\left(Z_{(k+1)}\right)\right) g\left(a_{(k) j}^{t}\right)\right)(j=1,2,3,4 ; t=u, l), \\
& v_{j}^{t}=g^{-1}\left(\sum_{k=1}^{n}\left(\mu\left(Z_{(k)}\right)-\mu\left(Z_{(k+1)}\right)\right) g\left(h_{j}\left(A_{(k)}^{t}\right)\right)\right)(j=1,2 ; t=u, l) .
\end{aligned}
$$

Then, we prove $b_{j}^{t}$ and $v_{j}^{t}$ satisfy the following conditions:

a) $0 \leq b_{j}^{t} \leq 1(j=1,2,3,4 ; t=u, l)$ and $0 \leq v_{j}^{t} \leq 1(j=1,2 ; t=u, l)$;

b) $b_{1}^{t} \leq b_{2}^{t} \leq b_{3}^{t} \leq b_{4}^{t}(t=u, l)$ and $v_{1}^{t} \leq v_{2}^{t}(t=u, l)$.

(1) We provide the proof of condition a). First, it is proved that $0 \leq b_{1}^{u} \leq 1$. Since $0 \leq a_{(k) 1}^{u} \leq 1$, and $g(t)$ is a monotonic decreasing function, the following is derived:

$$
g(1) \leq g\left(a_{(k) 1}^{u}\right) \leq g(0) .
$$

Then, since $0 \leq \mu\left(Z_{(k)}\right)-\mu\left(Z_{(k+1)}\right) \leq 1$, the following is derived:

$$
\left(\mu\left(Z_{(k)}\right)-\mu\left(Z_{(k+1)}\right)\right) g(1) \leq\left(\mu\left(Z_{(k)}\right)-\mu\left(Z_{(k+1)}\right)\right) g\left(a_{(k) 1}^{\mu}\right) \leq\left(\mu\left(Z_{(k)}\right)-\mu\left(Z_{(k+1)}\right)\right) g(0)
$$

and

$$
\sum_{k=1}^{n}\left(\mu\left(Z_{(k)}\right)-\mu\left(Z_{(k+1)}\right)\right) g(1) \leq \sum_{k=1}^{n}\left(\mu\left(Z_{(k)}\right)-\mu\left(Z_{(k+1)}\right)\right) g\left(a_{(k) 1}^{\mu}\right) \leq \sum_{k=1}^{n}\left(\mu\left(Z_{(k)}\right)-\mu\left(Z_{(k+1)}\right)\right) g(0) .
$$

In addition, since $\mu\left(Z_{(1)}\right)=1$ and $\mu\left(Z_{(n+1)}\right)=0$, the following is derived: 


$$
\sum_{k=1}^{n}\left(\mu\left(Z_{(k)}\right)-\mu\left(Z_{(k+1)}\right)\right)=\left(Z_{(1)}\right)-\mu\left(Z_{(n+1)}\right)=1 .
$$

Further, by Eqs. (ii)-(iii), the following is derived:

$$
g(1) \leq \sum_{k=1}^{n}\left(\mu\left(Z_{(k)}\right)-\mu\left(Z_{(k+1)}\right)\right) g\left(a_{(k) 1}^{\mu}\right) \leq g(0) .
$$

Finally, since $g^{-1}(t)$ is a monotonic decreasing function, by Eq. (iv), the following is derived:

$$
0=g^{-1}(g(0)) \leq g^{-1}\left(\sum_{k=1}^{n}\left(\mu\left(Z_{(k)}\right)-\mu\left(Z_{(k+1)}\right)\right) g\left(a_{(k) 1}^{\mu}\right)\right) \leq g^{-1}(g(1))=1 \text {, i.e., } 0 \leq b_{1}^{u} \leq 1 .
$$

Simultaneously, it can be proved that $0 \leq b_{1}^{l} \leq 1,0 \leq b_{j}^{t} \leq 1(j=2,3,4 ; t=u, l)$ and $0 \leq v_{j}^{t} \leq 1$ $(j=1,2 ; t=u, l)$.

(2) We provide the proof of condition b). First, it is proved that $b_{1}^{u} \leq b_{2}^{u}$. Since $a_{(k) 1}^{u} \leq a_{(k) 2}^{u}$, and $g(t)$ is a monotonic decreasing function, the following is derived:

$$
g\left(a_{(k) 2}^{u}\right) \leq g\left(a_{(k) 1}^{u}\right),
$$

Then, since $0 \leq \mu\left(Z_{(k)}\right)-\mu\left(Z_{(k+1)}\right) \leq 1$, by Eq. (v), the following is derived:

$$
\left(\mu\left(Z_{(k)}\right)-\mu\left(Z_{(k+1)}\right)\right) g\left(a_{(k) 2}^{u}\right) \leq\left(\mu\left(Z_{(k)}\right)-\mu\left(Z_{(k+1)}\right)\right) g\left(a_{(k) 1}^{u}\right)
$$

and

$$
\sum_{k=1}^{n}\left(\mu\left(Z_{(k)}\right)-\mu\left(Z_{(k+1)}\right)\right) g\left(a_{(k) 2}^{u}\right) \leq \sum_{k=1}^{n}\left(\mu\left(Z_{(k)}\right)-\mu\left(Z_{(k+1)}\right)\right) g\left(a_{(k) 1}^{u}\right) .
$$

Further, since $g^{-1}(t)$ is a monotonic decreasing function, by Eq. (vi), the following derived:

$$
g^{-1}\left(\sum_{k=1}^{n}\left(\mu\left(Z_{(k)}\right)-\mu\left(Z_{(k+1)}\right)\right) g\left(a_{(k) 1}^{u}\right)\right) \leq g^{-1}\left(\sum_{k=1}^{n}\left(\mu\left(Z_{(k)}\right)-\mu\left(Z_{(k+1)}\right)\right) g\left(a_{(k) 2}^{u}\right)\right) \text {, i.e., } b_{1}^{u} \leq b_{2}^{u} \text {. }
$$

Simultaneously, it can be proved that $b_{1}^{l} \leq b_{2}^{l}, b_{2}^{t} \leq b_{3}^{t} \leq b_{4}^{t}(t=u, l)$ and $v_{1}^{t} \leq v_{2}^{t}(t=u, l)$. Thus, by (1) and (2), the aggregation result of Eq. (17) is a trapezoidal IT2FN and Theorem 1 is proved true.

\section{APPENDIX 2}

Theorem 2. Let $\Lambda=\left\{A_{1}, A_{2}, \ldots, A_{n}\right\}$ and $\Lambda^{\prime}=\left\{A_{1}^{\prime}, A_{2}^{\prime}, \ldots, A_{n}^{\prime}\right\}$ be two sets of trapezoidal IT2FNs on $Z$, $B$ a trapezoidal IT2FN and $\xi>0$. The IT2FAC operator verifies the following properties:

(1) Idempotency: $\operatorname{IT} 2 F A C(A, A, \ldots, A)=A$, where $A=A_{k}(k=1,2, \ldots, n)$.

(2) Commutativity: IT2FAC $\left(A_{1}^{\prime}, A_{2}^{\prime}, \ldots, A_{n}^{\prime}\right)=\operatorname{IT} 2 F A C\left(A_{1}, A_{2}, \ldots, A_{n}\right)$, where $\left\{A_{1}^{\prime}, A_{2}^{\prime}, \ldots, A_{n}^{\prime}\right\}$ is any permutation of $\left\{A_{1}, A_{2}, \ldots, A_{n}\right\}$.

(3) Monotonicity: When $a_{(k) 1}^{\prime u} \leq a_{(k) 1}^{u}, a_{(k) 2}^{\prime u} \leq a_{(k) 2}^{u}, a_{(k) 3}^{\prime u} \leq a_{(k) 3}^{u}, a_{(k) 4}^{\prime u} \leq a_{(k) 4}^{u}, h_{1}^{u}\left(A_{(k)}^{\prime}\right) \leq h_{1}^{u}\left(A_{(k)}\right)$, $h_{2}^{u}\left(A_{(k)}^{\prime}\right) \leq h_{2}^{u}\left(A_{(k)}\right), \quad a_{(k) 1}^{\prime l} \leq a_{(k) 1}^{l}, \quad a_{(k) 2}^{\prime l} \leq a_{(k) 2}^{l}, \quad a_{(k) 3}^{\prime l} \leq a_{(k) 3}^{l}, \quad a_{(k) 4}^{\prime l} \leq a_{(k) 4}^{l}, \quad h_{1}^{l}\left(A_{(k)}^{\prime}\right) \leq h_{1}^{l}\left(A_{(k)}\right)$, $h_{2}^{l}\left(A_{(k)}^{\prime}\right) \leq h_{2}^{l}\left(A_{(k)}\right)$, it is $\operatorname{IT} 2 F A C\left(A_{1}^{\prime}, A_{2}^{\prime}, \ldots, A_{n}^{\prime}\right) \leq \operatorname{IT} 2 F A C\left(A_{1}, A_{2}, \ldots, A_{n}\right)$, where $A_{(k)}^{\prime}$ is the $k$-th 
smallest value of the set $\Lambda^{\prime}$.

(4) Boundedness: $A^{-} \leq I T 2 F A C\left(A_{1}, A_{2}, \ldots, A_{n}\right) \leq A^{+}$, where

$$
\begin{aligned}
A^{-}= & \left(\left(\min _{k}\left(a_{k 1}^{u}\right), \min _{k}\left(a_{k 2}^{u}\right), \min _{k}\left(a_{k 3}^{u}\right), \min _{k}\left(a_{k 4}^{u}\right) ; \min _{k}\left(h_{1}\left(A_{k}^{u}\right)\right), \min _{k}\left(h_{2}\left(A_{k}^{u}\right)\right)\right),\right. \\
& \left.\left(\min _{k}\left(a_{k 1}^{l}\right), \min _{k}\left(a_{k 2}^{l}\right), \min _{k}\left(a_{k 3}^{l}\right), \min _{k}\left(a_{k 4}^{l}\right) ; \min _{k}\left(h_{1}\left(A_{k}^{l}\right)\right), \min _{k}\left(h_{2}\left(A_{k}^{l}\right)\right)\right)\right)
\end{aligned}
$$

and

$$
\begin{aligned}
& A^{+}=\left(\left(\max _{k}\left(a_{k 1}^{u}\right), \max _{k}\left(a_{k 2}^{u}\right), \max _{k}\left(a_{k 3}^{u}\right), \max _{k}\left(a_{k 4}^{u}\right) ; \max _{k}\left(h_{1}\left(A_{k}^{u}\right)\right), \max _{k}\left(h_{2}\left(A_{k}^{u}\right)\right)\right),\right. \\
&\left.\left(\max _{k}\left(a_{k 1}^{l}\right), \max _{k}\left(a_{k 2}^{l}\right), \max _{k}\left(a_{k 3}^{l}\right), \max _{k}\left(a_{k 4}^{l}\right) ; \max _{k}\left(h_{1}\left(A_{k}^{l}\right)\right), \max _{k}\left(h_{2}\left(A_{k}^{l}\right)\right)\right)\right) . \\
& \text { (5) } \operatorname{IT} 2 F A C\left(A_{1}^{\xi} \otimes B, A_{2}^{\xi} \otimes B, \ldots, A_{n}^{\xi} \otimes B\right)=\left(\operatorname{IT} 2 F A C\left(A_{1}, A_{2}, \ldots, A_{n}\right)\right)^{\xi} \otimes B . \\
& \text { (6) } \operatorname{IT} 2 \text { FAC }\left(A_{1} \otimes A_{1}^{\prime}, A_{2} \otimes A_{2}^{\prime}, \ldots, A_{n} \otimes A_{n}^{\prime}\right)=\operatorname{IT} 2 F A C\left(A_{1}, A_{2}, \ldots, A_{n}\right) \otimes I T 2 F A C\left(A_{1}^{\prime}, A_{2}^{\prime}, \ldots, A_{n}^{\prime}\right) .
\end{aligned}
$$

Proof. (1) Since $A_{k}=A=\left(\left(a_{1}^{u}, a_{2}^{u}, a_{3}^{u}, a_{4}^{u} ; h_{1}\left(A^{u}\right), h_{2}\left(A^{u}\right)\right),\left(a_{1}^{l}, a_{2}^{l}, a_{3}^{l}, a_{4}^{l} ; h_{1}\left(A^{l}\right), h_{2}\left(A^{l}\right)\right)\right)$ for $\quad$ all $k(k=1,2, \ldots, n)$ and $\sum_{k=1}^{n}\left(\mu\left(Z_{(k)}\right)-\mu\left(Z_{(k+1)}\right)\right)=1$, according to Theorem $\mathbf{1}$, the following is derived:

$$
\begin{aligned}
& \operatorname{IT} 2 \operatorname{FAC}\left(A_{1}, A_{2}, \ldots, A_{n}\right) \\
& =\left(\left(g^{-1}\left(\sum_{k=1}^{n}\left(\mu\left(Z_{(k)}\right)-\mu\left(Z_{(k+1)}\right)\right) g\left(a_{(k) 1}^{u}\right)\right), g^{-1}\left(\sum_{k=1}^{n}\left(\mu\left(Z_{(k)}\right)-\mu\left(Z_{(k+1)}\right)\right) g\left(a_{(k) 2}^{u}\right)\right)\right.\right. \text {, } \\
& g^{-1}\left(\sum_{k=1}^{n}\left(\mu\left(Z_{(k)}\right)-\mu\left(Z_{(k+1)}\right)\right) g\left(a_{(k) 3}^{u}\right)\right), g^{-1}\left(\sum_{k=1}^{n}\left(\mu\left(Z_{(k)}\right)-\mu\left(Z_{(k+1)}\right)\right) g\left(a_{(k) 4}^{u}\right)\right) ; \\
& \left.g^{-1}\left(\sum_{k=1}^{n}\left(\mu\left(Z_{(k)}\right)-\mu\left(Z_{(k+1)}\right)\right) g\left(h_{1}\left(A_{(k)}^{u}\right)\right)\right), g^{-1}\left(\sum_{k=1}^{n}\left(\mu\left(Z_{(k)}\right)-\mu\left(Z_{(k+1)}\right)\right) g\left(h_{2}\left(A_{(k)}^{u}\right)\right)\right)\right) \text {, } \\
& \left(g^{-1}\left(\sum_{k=1}^{n}\left(\mu\left(Z_{(k)}\right)-\mu\left(Z_{(k+1)}\right)\right) g\left(a_{(k) 1}^{l}\right)\right), g^{-1}\left(\sum_{k=1}^{n}\left(\mu\left(Z_{(k)}\right)-\mu\left(Z_{(k+1)}\right)\right) g\left(a_{(k) 2}^{l}\right)\right),\right. \\
& g^{-1}\left(\sum_{k=1}^{n}\left(\mu\left(Z_{(k)}\right)-\mu\left(Z_{(k+1)}\right)\right) g\left(a_{(k) 3}^{l}\right)\right), g^{-1}\left(\sum_{k=1}^{n}\left(\mu\left(Z_{(k)}\right)-\mu\left(Z_{(k+1)}\right)\right) g\left(a_{(k) 4}^{l}\right)\right) ; \\
& \left.\left.g^{-1}\left(\sum_{k=1}^{n}\left(\mu\left(Z_{(k)}\right)-\mu\left(Z_{(k+1)}\right)\right) g\left(h_{1}\left(A_{(k)}^{l}\right)\right)\right), g^{-1}\left(\sum_{k=1}^{n}\left(\mu\left(Z_{(k)}\right)-\mu\left(Z_{(k+1)}\right)\right) g\left(h_{2}\left(A_{(k)}^{l}\right)\right)\right)\right)\right) \\
& =\left(\left(g^{-1}\left(g\left(a_{1}^{u}\right) \sum_{k=1}^{n}\left(\mu\left(Z_{(k)}\right)-\mu\left(Z_{(k+1)}\right)\right)\right), g^{-1}\left(g\left(a_{2}^{u}\right) \sum_{k=1}^{n}\left(\mu\left(Z_{(k)}\right)-\mu\left(Z_{(k+1)}\right)\right)\right),\right.\right. \\
& g^{-1}\left(g\left(a_{3}^{u}\right) \sum_{k=1}^{n}\left(\mu\left(Z_{(k)}\right)-\mu\left(Z_{(k+1)}\right)\right)\right), g^{-1}\left(g\left(a_{4}^{u}\right) \sum_{k=1}^{n}\left(\mu\left(Z_{(k)}\right)-\mu\left(Z_{(k+1)}\right)\right)\right) ; \\
& \left.g^{-1}\left(g\left(h_{1}\left(A^{u}\right)\right) \sum_{k=1}^{n}\left(\mu\left(Z_{(k)}\right)-\mu\left(Z_{(k+1)}\right)\right)\right), g^{-1}\left(g\left(h_{2}\left(A^{u}\right)\right) \sum_{k=1}^{n}\left(\mu\left(Z_{(k)}\right)-\mu\left(Z_{(k+1)}\right)\right)\right)\right) \text {, } \\
& \left(g^{-1}\left(g\left(a_{1}^{l}\right) \sum_{k=1}^{n}\left(\mu\left(Z_{(k)}\right)-\mu\left(Z_{(k+1)}\right)\right)\right), g^{-1}\left(g\left(a_{2}^{l}\right) \sum_{k=1}^{n}\left(\mu\left(Z_{(k)}\right)-\mu\left(Z_{(k+1)}\right)\right)\right),\right. \\
& g^{-1}\left(g\left(a_{3}^{l}\right) \sum_{k=1}^{n}\left(\mu\left(Z_{(k)}\right)-\mu\left(Z_{(k+1)}\right)\right)\right), g^{-1}\left(g\left(a_{4}^{l}\right) \sum_{k=1}^{n}\left(\mu\left(Z_{(k)}\right)-\mu\left(Z_{(k+1)}\right)\right)\right) ;
\end{aligned}
$$




$$
\begin{aligned}
& \left.\left.g^{-1}\left(g\left(h_{1}\left(A^{l}\right)\right) \sum_{k=1}^{n}\left(\mu\left(Z_{(k)}\right)-\mu\left(Z_{(k+1)}\right)\right)\right), g^{-1}\left(g\left(h_{2}\left(A^{l}\right)\right) \sum_{k=1}^{n}\left(\mu\left(Z_{(k)}\right)-\mu\left(Z_{(k+1)}\right)\right)\right)\right)\right) \\
& =\left(\left(a_{1}^{u}, a_{2}^{u}, a_{3}^{u}, a_{3}^{u} ; h_{1}\left(A^{u}\right), h_{2}\left(A^{u}\right)\right),\left(a_{1}^{l}, a_{2}^{l}, a_{3}^{l}, a_{4}^{l} ; h_{1}\left(A^{l}\right), h_{2}\left(A^{l}\right)\right)\right)=A .
\end{aligned}
$$

(2) Since $\left\{A_{1}^{\prime}, A_{2}^{\prime}, \ldots, A_{n}^{\prime}\right\}$ is any permutation of $\left\{A_{1}, A_{2}, \ldots, A_{n}\right\}$, by Definition 7, the following is derived: $\operatorname{IT} 2 F A C\left(A_{1}^{\prime}, A_{2}^{\prime}, \ldots, A_{n}^{\prime}\right)=\operatorname{IT} 2 F A C\left(A_{1}, A_{2}, \ldots, A_{n}\right)$.

(3) Let IT2FAC $\left(A_{1}, A_{2}, \ldots, A_{n}\right)=\left(\left(a_{1}^{u}, a_{2}^{u}, a_{3}^{u}, a_{4}^{u} ; h_{1}\left(A_{1}^{u}\right), h_{1}\left(A_{2}^{u}\right)\right),\left(a_{1}^{l}, a_{2}^{l}, a_{3}^{l}, a_{4}^{l} ; h_{1}\left(A_{1}^{l}\right), h_{1}\left(A_{2}^{l}\right)\right)\right)$

and $\operatorname{IT} 2 F A C\left(A_{1}^{\prime}, A_{2}^{\prime}, \ldots, A_{n}^{\prime}\right)=\left(\left(a_{1}^{\prime \prime}, a_{2}^{\prime \prime}, a_{3}^{\prime \prime}, a_{4}^{\prime \prime} ; h_{1}\left(A_{1}^{\prime \prime}\right), h_{1}\left(A_{2}^{\prime \prime}\right)\right),\left(a_{1}^{\prime l}, a_{2}^{\prime l}, a_{3}^{\prime l}, a_{4}^{\prime l} ; h_{1}\left(A_{1}^{\prime l}\right), h_{1}\left(A_{2}^{\prime l}\right)\right)\right)$.

i) First, it is proved that $a_{1}^{\prime \prime} \leq a_{1}^{u}$. Since $g(t)$ is a monotonic decreasing function and $a_{(k) 1}^{\prime \prime} \leq a_{(k) 1}^{u}$, it is $g\left(a_{(k) 1}^{\prime \prime}\right) \geq g\left(a_{(k) 1}^{u}\right)$. Then, since $\mu\left(Z_{(k)}\right)-\mu\left(Z_{(k+1)}\right) \geq 0(k=1,2, \ldots, n)$, the following is derived:

$$
\left(\mu\left(Z_{(k)}\right)-\mu\left(Z_{(k+1)}\right)\right) g\left(a_{(k) 1}^{\prime \prime}\right) \geq\left(\mu\left(Z_{(k)}\right)-\mu\left(Z_{(k+1)}\right)\right) g\left(a_{(k) 1}^{u}\right)
$$

and

$$
\sum_{k=1}^{n}\left(\mu\left(Z_{(k)}\right)-\mu\left(Z_{(k+1)}\right)\right) g\left(a_{(k) 1}^{\prime u}\right) \geq \sum_{k=1}^{n}\left(\mu\left(Z_{(k)}\right)-\mu\left(Z_{(k+1)}\right)\right) g\left(a_{(k) 1}^{u}\right) .
$$

Additionally, since $g^{-1}(t)$ is a monotonic decreasing function, the following is derived:

$$
g^{-1}\left(\sum_{k=1}^{n}\left(\mu\left(Z_{(k)}\right)-\mu\left(Z_{(k+1)}\right)\right) g\left(a_{(k) 1}^{\prime \prime}\right)\right) \leq g^{-1}\left(\sum_{k=1}^{n}\left(\mu\left(Z_{(k)}\right)-\mu\left(Z_{(k+1)}\right)\right) g\left(a_{(k) 1}^{u}\right)\right) \text {, i.e., } a_{1}^{\prime \prime} \leq a_{1}^{u} \text {. }
$$

ii) Similar to i), it is proved that:

$$
a_{1}^{\prime l} \leq a_{1}^{l}, a_{j}^{\prime t} \leq a_{j}^{t}(j=2,3,4 ; t=u, l), h_{j}\left(A_{(k)}^{t}\right) \leq h_{j}\left(A_{(k)}^{t}\right)(j=1,2 ; t=u, l) .
$$

iii) Further, by Definition 3, the following is derived:

$$
R\left(\operatorname{IT} 2 F A C\left(A_{1}^{\prime}, A_{2}^{\prime}, \ldots, A_{n}^{\prime}\right)\right) \leq R\left(\operatorname{IT} 2 F A C\left(A_{1}, A_{2}, \ldots, A_{n}\right)\right) .
$$

Thus, by Definition 4, it is $\operatorname{IT} 2 F A C\left(A_{1}^{\prime}, A_{2}^{\prime}, \ldots, A_{n}^{\prime}\right) \leq \operatorname{IT} 2 F A C\left(A_{1}, A_{2}, \ldots, A_{n}\right)$.

(4) Since

$$
\begin{aligned}
A^{-}= & \left(\left(\min _{k}\left(a_{k 1}^{u}\right), \min _{k}\left(a_{k 2}^{u}\right), \min _{k}\left(a_{k 3}^{u}\right), \min _{k}\left(a_{k 4}^{u}\right) ; \min _{k}\left(h_{1}\left(A_{k}^{u}\right)\right), \min _{k}\left(h_{2}\left(A_{k}^{u}\right)\right)\right),\right. \\
& \left.\left(\min _{k}\left(a_{k 1}^{l}\right), \min _{k}\left(a_{k 2}^{l}\right), \min _{k}\left(a_{k 3}^{l}\right), \min _{k}\left(a_{k 4}^{l}\right) ; \min _{k}\left(h_{1}\left(A_{k}^{l}\right)\right), \min _{k}\left(h_{2}\left(A_{k}^{l}\right)\right)\right)\right)
\end{aligned}
$$

(6)id

$$
\begin{array}{r}
A^{+}=\left(\left(\max _{k}\left(a_{k 1}^{u}\right), \max _{k}\left(a_{k 2}^{u}\right), \max _{k}\left(a_{k 3}^{u}\right), \max _{k}\left(a_{k 4}^{u}\right) ; \max _{k}\left(h_{1}\left(A_{k}^{u}\right)\right), \max _{k}\left(h_{2}\left(A_{k}^{u}\right)\right)\right),\right. \\
\left.\left(\max _{k}\left(a_{k 1}^{l}\right), \max _{k}\left(a_{k 2}^{l}\right), \max _{k}\left(a_{k 3}^{l}\right), \max _{k}\left(a_{k 4}^{l}\right) ; \max _{k}\left(h_{1}\left(A_{k}^{l}\right)\right), \max _{k}\left(h_{2}\left(A_{k}^{l}\right)\right)\right)\right),
\end{array}
$$

by the monotonicity property of the proposed IT2FAC operator, the following is derived:

$$
\operatorname{IT} 2 F A C\left(A^{-}, A^{-}, \ldots, A^{-}\right) \leq \operatorname{IT} 2 F A C\left(A_{1}, A_{2}, \ldots, A_{n}\right) \leq I T 2 F A C\left(A^{+}, A^{+}, \ldots, A^{+}\right) .
$$

Then, by the idempotency property of the proposed IT2FAC operator, the following is derived: $A^{-}=\operatorname{IT} 2 F A C\left(A^{-}, A^{-}, \ldots, A^{-}\right)$and $A^{+}=\operatorname{IT} 2 F A C\left(A^{+}, A^{+}, \ldots, A^{+}\right)$.

Thus, we conclude that: $A^{-} \leq \operatorname{IT} 2 F A C\left(A_{1}, A_{2}, \ldots, A_{n}\right) \leq A^{+}$. 
(5) Since $B=\left(B^{u}, B^{l}\right)$ is a trapezoidal IT2FN and $\xi>0$, by Eqs. (6)-(7), the following is derived:

$$
\begin{aligned}
& \left(A_{k}\right)^{\xi} \otimes B \\
= & \left(\left(g^{-1}\left(\xi g\left(a_{k 1}^{u}\right)+g\left(b_{1}^{u}\right)\right), g^{-1}\left(\xi g\left(a_{k 2}^{u}\right)+g\left(b_{2}^{u}\right)\right), g^{-1}\left(\xi g\left(a_{k 3}^{u}\right)+g\left(b_{3}^{u}\right)\right),\right.\right. \\
& \left.g^{-1}\left(\xi g\left(a_{k 4}^{u}\right)+g\left(b_{4}^{u}\right)\right) ; g^{-1}\left(\xi g\left(h_{1}\left(A_{k}^{u}\right)\right)+g\left(h_{1}\left(B^{u}\right)\right)\right), g^{-1}\left(\xi g\left(h_{2}\left(A_{k}^{u}\right)\right)+g\left(h_{2}\left(B^{u}\right)\right)\right)\right), \\
& \left(g^{-1}\left(\xi g\left(a_{k 1}^{l}\right)+g\left(b_{1}^{l}\right)\right), g^{-1}\left(\xi g\left(a_{k 2}^{l}\right)+g\left(b_{2}^{l}\right)\right), g^{-1}\left(\xi g\left(a_{k 3}^{l}\right)+g\left(b_{3}^{l}\right)\right),\right. \\
& \left.\left.g^{-1}\left(\xi g\left(a_{k 4}^{l}\right)+g\left(b_{4}^{l}\right)\right) ; g^{-1}\left(\xi g\left(h_{1}\left(A_{k}^{l}\right)\right)+g\left(h_{1}\left(B^{l}\right)\right)\right), g^{-1}\left(\xi g\left(h_{2}\left(A_{k}^{l}\right)\right)+g\left(h_{2}\left(B^{l}\right)\right)\right)\right)\right) .
\end{aligned}
$$

for all $k(k=1,2, \ldots, n)$.

Then, by Theorem 1 and $\sum_{k=1}^{n}\left(\mu\left(Z_{(k)}\right)-\mu\left(Z_{(k+1)}\right)\right)=1$, the following is derived:

$\operatorname{IT} 2 F A C\left(A_{1}^{\xi} \otimes B, A_{2}^{\xi} \otimes B, \ldots, A_{n}^{\xi} \otimes B\right)$

$$
\begin{aligned}
& =\left(\left(g^{-1}\left(\sum_{k=1}^{n}\left(\mu\left(Z_{(k)}\right)-\mu\left(Z_{(k+1)}\right)\right) g\left(g^{-1}\left(\xi g\left(a_{(k) 1}^{u}\right)+g\left(b_{1}^{u}\right)\right)\right)\right),\right.\right. \\
& g^{-1}\left(\sum_{k=1}^{n}\left(\mu\left(Z_{(k)}\right)-\mu\left(Z_{(k+1)}\right)\right) g\left(g^{-1}\left(\xi g\left(a_{(k) 2}^{u}+g\left(b_{2}^{u}\right)\right)\right)\right),\right. \\
& g^{-1}\left(\sum_{k=1}^{n}\left(\mu\left(Z_{(k)}\right)-\mu\left(Z_{(k+1)}\right)\right) g\left(g^{-1}\left(\xi g\left(a_{(k) 3}^{u}\right)+g\left(b_{3}^{u}\right)\right)\right)\right), \\
& g^{-1}\left(\sum_{k=1}^{n}\left(\mu\left(Z_{(k)}\right)-\mu\left(Z_{(k+1)}\right)\right) g\left(g^{-1}\left(\xi g\left(a_{(k) 4}^{u}\right)+g\left(b_{4}^{u}\right)\right)\right)\right) ; \\
& g^{-1}\left(\sum_{k=1}^{n}\left(\mu\left(Z_{(k)}\right)-\mu\left(Z_{(k+1)}\right)\right) g\left(g^{-1}\left(\xi g\left(h_{1}\left(A_{(k)}^{u}\right)\right)+g\left(h_{1}\left(B^{u}\right)\right)\right)\right)\right), \\
& g^{-1}\left(\sum_{k=1}^{n}\left(\mu\left(Z_{(k)}\right)-\mu\left(Z_{(k+1)}\right)\right) g\left(g^{-1}\left(\xi g\left(h_{2}\left(A_{(k)}^{u}\right)\right)+g\left(h_{2}\left(B^{u}\right)\right)\right)\right)\right), \\
& \left(g^{-1}\left(\sum_{k=1}^{n}\left(\mu\left(Z_{(k)}\right)-\mu\left(Z_{(k+1)}\right)\right) g\left(g^{-1}\left(\xi g\left(a_{(k) 1}^{l}\right)+g\left(b_{1}^{l}\right)\right)\right)\right),\right. \\
& g^{-1}\left(\sum_{k=1}^{n}\left(\mu\left(Z_{(k)}\right)-\mu\left(Z_{(k+1)}\right)\right) g\left(g^{-1}\left(\xi g\left(a_{(k) 2}^{l}\right)+g\left(b_{2}^{l}\right)\right)\right)\right), \\
& g^{-1}\left(\sum_{k=1}^{n}\left(\mu\left(Z_{(k)}\right)-\mu\left(Z_{(k+1)}\right)\right) g\left(g^{-1}\left(\xi g\left(a_{(k) 3}^{l}\right)+g\left(b_{3}^{l}\right)\right)\right)\right), \\
& g^{-1}\left(\sum_{k=1}^{n}\left(\mu\left(Z_{(k)}\right)-\mu\left(Z_{(k+1)}\right)\right) g\left(g^{-1}\left(\xi g\left(a_{(k) 4}^{l}\right)+g\left(b_{4}^{l}\right)\right)\right)\right) ; \\
& g^{-1}\left(\sum_{k=1}^{n}\left(\mu\left(Z_{(k)}\right)-\mu\left(Z_{(k+1)}\right)\right) g\left(g^{-1}\left(\xi g\left(h_{1}\left(A_{(k)}^{l}\right)\right)+g\left(h_{1}\left(B^{l}\right)\right)\right)\right)\right), \\
& \left.\left.g^{-1}\left(\sum_{k=1}^{n}\left(\mu\left(Z_{(k)}\right)-\mu\left(Z_{(k+1)}\right)\right) g\left(g^{-1}\left(\xi g\left(h_{2}\left(A_{(k)}^{l}\right)\right)+g\left(h_{2}\left(B^{l}\right)\right)\right)\right)\right)\right)\right) \\
& =\left(\left(g^{-1}\left(\sum_{k=1}^{n}\left(\mu\left(Z_{(k)}\right)-\mu\left(Z_{(k+1)}\right)\right) \xi g\left(a_{(k) 1}^{u}\right)+g\left(b_{1}^{u}\right)\right), g^{-1}\left(\sum_{k=1}^{n}\left(\mu\left(Z_{(k)}\right)-\mu\left(Z_{(k+1)}\right)\right) \xi g\left(a_{(k) 2}^{u}\right)+g\left(b_{2}^{u}\right)\right)\right.\right. \text {, } \\
& g^{-1}\left(\sum_{k=1}^{n}\left(\mu\left(Z_{(k)}\right)-\mu\left(Z_{(k+1)}\right)\right) \xi g\left(a_{(k) 3}^{u}\right)+g\left(b_{3}^{u}\right)\right), g^{-1}\left(\sum_{k=1}^{n}\left(\mu\left(Z_{(k)}\right)-\mu\left(Z_{(k+1)}\right)\right) \xi g\left(a_{(k) 4}^{u}\right)+g\left(b_{4}^{u}\right)\right) ;
\end{aligned}
$$




$$
\begin{aligned}
& g^{-1}\left(\sum_{k=1}^{n}\left(\mu\left(Z_{(k)}\right)-\mu\left(Z_{(k+1)}\right)\right) \xi g\left(h_{1}\left(A_{(k)}^{u}\right)\right)+g\left(h_{1}\left(B^{u}\right)\right)\right), \\
& \left.g^{-1}\left(\sum_{k=1}^{n}\left(\mu\left(Z_{(k)}\right)-\mu\left(Z_{(k+1)}\right)\right) \xi g\left(h_{2}\left(A_{(k)}^{u}\right)\right)+g\left(h_{2}\left(B^{u}\right)\right)\right)\right), \\
& \left(g^{-1}\left(\sum_{k=1}^{n}\left(\mu\left(Z_{(k)}\right)-\mu\left(Z_{(k+1)}\right)\right) \xi g\left(a_{(k) 1}^{l}\right)+g\left(b_{1}^{l}\right)\right), g^{-1}\left(\sum_{k=1}^{n}\left(\mu\left(Z_{(k)}\right)-\mu\left(Z_{(k+1)}\right)\right) \xi g\left(a_{(k) 2}^{l}\right)+g\left(b_{2}^{l}\right)\right),\right. \\
& g^{-1}\left(\sum_{k=1}^{n}\left(\mu\left(Z_{(k)}\right)-\mu\left(Z_{(k+1)}\right)\right) \xi g\left(a_{(k) 3}^{l}\right)+g\left(b_{3}^{l}\right)\right), g^{-1}\left(\sum_{k=1}^{n}\left(\mu\left(Z_{(k)}\right)-\mu\left(Z_{(k+1)}\right)\right) \xi g\left(a_{(k) 4}^{l}\right)+g\left(b_{4}^{l}\right)\right) ; \\
& g^{-1}\left(\sum_{k=1}^{n}\left(\mu\left(Z_{(k)}\right)-\mu\left(Z_{(k+1)}\right)\right) \xi g\left(h_{1}\left(A_{(k)}^{l}\right)\right)+g\left(h_{1}\left(B^{l}\right)\right)\right) \text {, } \\
& \left.\left.g^{-1}\left(\sum_{k=1}^{n}\left(\mu\left(Z_{(k)}\right)-\mu\left(Z_{(k+1)}\right)\right) \xi g\left(h_{2}\left(A_{(k)}^{l}\right)\right)+g\left(h_{2}\left(B^{l}\right)\right)\right)\right)\right) \\
& =\left(\left(g^{-1}\left(\xi \sum_{k=1}^{n}\left(\mu\left(Z_{(k)}\right)-\mu\left(Z_{(k+1)}\right)\right) g\left(a_{(k) 1}^{u}\right)\right), g^{-1}\left(\xi \sum_{k=1}^{n}\left(\mu\left(Z_{(k)}\right)-\mu\left(Z_{(k+1)}\right)\right) g\left(a_{(k) 2}^{u}\right)\right),\right.\right. \\
& g^{-1}\left(\xi \sum_{k=1}^{n}\left(\mu\left(Z_{(k)}\right)-\mu\left(Z_{(k+1)}\right)\right) g\left(a_{(k) 3}^{u}\right)\right), g^{-1}\left(\xi \sum_{k=1}^{n}\left(\mu\left(Z_{(k)}\right)-\mu\left(Z_{(k+1)}\right)\right) g\left(a_{(k) 4}^{u}\right)\right) ; \\
& \left.g^{-1}\left(\xi \sum_{k=1}^{n}\left(\mu\left(Z_{(k)}\right)-\mu\left(Z_{(k+1)}\right)\right) g\left(h_{1}\left(A_{(k)}^{u}\right)\right)\right), g^{-1}\left(\xi \sum_{k=1}^{n}\left(\mu\left(Z_{(k)}\right)-\mu\left(Z_{(k+1)}\right)\right) g\left(h_{2}\left(A_{(k)}^{u}\right)\right)\right)\right), \\
& \left(g^{-1}\left(\xi \sum_{k=1}^{n}\left(\mu\left(Z_{(k)}\right)-\mu\left(Z_{(k+1)}\right)\right) g\left(a_{(k) 1}^{l}\right)\right), g^{-1}\left(\xi \sum_{k=1}^{n}\left(\mu\left(Z_{(k)}\right)-\mu\left(Z_{(k+1)}\right)\right) g\left(a_{(k) 2}^{l}\right)\right),\right. \\
& g^{-1}\left(\xi \sum_{k=1}^{n}\left(\mu\left(Z_{(k)}\right)-\mu\left(Z_{(k+1)}\right)\right) g\left(a_{(k) 3}^{l}\right)\right), g^{-1}\left(\xi \sum_{k=1}^{n}\left(\mu\left(Z_{(k)}\right)-\mu\left(Z_{(k+1)}\right)\right) g\left(a_{(k) 4}^{l}\right)\right) ; \\
& \left.\left.g^{-1}\left(\xi \sum_{k=1}^{n}\left(\mu\left(Z_{(k)}\right)-\mu\left(Z_{(k+1)}\right)\right) g\left(h_{1}\left(A_{(k)}^{l}\right)\right)\right), g^{-1}\left(\xi \sum_{k=1}^{n}\left(\mu\left(Z_{(k)}\right)-\mu\left(Z_{(k+1)}\right)\right) g\left(h_{2}\left(A_{(k)}^{l}\right)\right)\right)\right)\right) \\
& \otimes\left(\left(b_{1}^{u}, b_{2}^{u}, b_{3}^{u}, b_{4}^{u} ; h_{1}\left(B^{u}\right), h_{2}\left(B^{u}\right)\right),\left(b_{1}^{l}, b_{2}^{l}, b_{3}^{l}, b_{4}^{l} ; h_{1}\left(B^{l}\right), h_{2}\left(B^{l}\right)\right)\right) \\
& =\left(\operatorname{IT} 2 F A C\left(A_{1}, A_{2}, \ldots, A_{n}\right)\right)^{\xi} \otimes B
\end{aligned}
$$

(6) By Eq. (6), the following is derived:

$$
\begin{array}{r}
A_{1} \otimes A_{1}^{\prime}=\left(\left(g^{-1}\left(g\left(a_{11}^{u}\right)+g\left(a_{11}^{\prime u}\right)\right), g^{-1}\left(g\left(a_{12}^{u}\right)+g\left(a_{12}^{\prime u}\right)\right), g^{-1}\left(g\left(a_{13}^{u}\right)+g\left(a_{13}^{\prime u}\right)\right), g^{-1}\left(g\left(a_{14}^{u}\right)+g\left(a_{14}^{\prime u}\right)\right) ;\right.\right. \\
\left.g^{-1}\left(g\left(h_{1}\left(A_{1}^{u}\right)\right)+g\left(h_{1}\left(A_{1}^{\prime \prime}\right)\right)\right), g^{-1}\left(g\left(h_{2}\left(A_{1}^{u}\right)\right)+g\left(h_{2}\left(A_{1}^{\prime \prime}\right)\right)\right)\right), \\
\left(g^{-1}\left(g\left(a_{11}^{l}\right)+g\left(a_{11}^{\prime l}\right)\right), g^{-1}\left(g\left(a_{12}^{l}\right)+g\left(a_{12}^{\prime l}\right)\right), g^{-1}\left(g\left(a_{13}^{l}\right)+g\left(a_{13}^{\prime l}\right)\right), g^{-1}\left(g\left(a_{14}^{l}\right)+g\left(a_{14}^{\prime l}\right)\right) ;\right. \\
\left.\left.g^{-1}\left(g\left(h_{1}\left(A_{1}^{l}\right)\right)+g\left(h_{1}\left(A_{1}^{\prime l}\right)\right)\right), g^{-1}\left(g\left(h_{2}\left(A_{1}^{l}\right)\right)+g\left(h_{2}\left(A_{1}^{\prime l}\right)\right)\right)\right)\right) .
\end{array}
$$

Then, by Theorem 1, the following is derived:

$$
\begin{aligned}
& \text { IT } 2 F A C\left(A_{1} \otimes A_{1}^{\prime}, A_{2} \otimes A_{2}^{\prime}, \ldots, A_{n} \otimes A_{n}^{\prime}\right) \\
& =\left(\left(g^{-1}\left(\sum_{k=1}^{n}\left(\mu\left(Z_{(k)}\right)-\mu\left(Z_{(k+1)}\right)\right) g\left(g^{-1}\left(g\left(a_{(k) 1}^{u}\right)+g\left(a_{(k) 1}^{\prime u}\right)\right)\right)\right),\right.\right. \\
& g^{-1}\left(\sum_{k=1}^{n}\left(\mu\left(Z_{(k)}\right)-\mu\left(Z_{(k+1)}\right)\right) g\left(g^{-1}\left(g\left(a_{(k) 2}^{u}\right)+g\left(a_{(k) 2}^{\prime u}\right)\right)\right)\right), \\
& g^{-1}\left(\sum_{k=1}^{n}\left(\mu\left(Z_{(k)}\right)-\mu\left(Z_{(k+1)}\right)\right) g\left(g^{-1}\left(g\left(a_{(k) 3}^{u}\right)+g\left(a_{(k) 3}^{\prime u}\right)\right)\right)\right), \\
& g^{-1}\left(\sum_{k=1}^{n}\left(\mu\left(Z_{(k)}\right)-\mu\left(Z_{(k+1)}\right)\right) g\left(g^{-1}\left(g\left(a_{(k) 4}^{u}\right)+g\left(a_{(k) 4}^{\prime u}\right)\right)\right)\right) ;
\end{aligned}
$$




$$
\begin{aligned}
& g^{-1}\left(\sum_{k=1}^{n}\left(\mu\left(Z_{(k)}\right)-\mu\left(Z_{(k+1)}\right)\right) g\left(g^{-1}\left(g\left(h_{1}\left(A_{(k)}^{u}\right)\right)+g\left(h_{1}\left(A_{(k)}^{\prime \prime}\right)\right)\right)\right)\right), \\
& \left.g^{-1}\left(\sum_{k=1}^{n}\left(\mu\left(Z_{(k)}\right)-\mu\left(Z_{(k+1)}\right)\right) g\left(g^{-1}\left(g\left(h_{2}\left(A_{(k)}^{u}\right)\right)+g\left(h_{2}\left(A_{(k)}^{\prime u}\right)\right)\right)\right)\right)\right) \text {, } \\
& \left(g^{-1}\left(\sum_{k=1}^{n}\left(\mu\left(Z_{(k)}\right)-\mu\left(Z_{(k+1)}\right)\right) g\left(g^{-1}\left(g\left(a_{(k) 1}^{l}\right)+g\left(a_{(k) 1}^{\prime l}\right)\right)\right)\right),\right. \\
& g^{-1}\left(\sum_{k=1}^{n}\left(\mu\left(Z_{(k)}\right)-\mu\left(Z_{(k+1)}\right)\right) g\left(g^{-1}\left(g\left(a_{(k) 2}^{l}\right)+g\left(a_{(k) 2}^{\prime l}\right)\right)\right)\right), \\
& g^{-1}\left(\sum_{k=1}^{n}\left(\mu\left(Z_{(k)}\right)-\mu\left(Z_{(k+1)}\right)\right) g\left(g^{-1}\left(g\left(a_{(k) 3}^{l}\right)+g\left(a_{(k) 3}^{\prime l}\right)\right)\right)\right), \\
& g^{-1}\left(\sum_{k=1}^{n}\left(\mu\left(Z_{(k)}\right)-\mu\left(Z_{(k+1)}\right)\right) g\left(g^{-1}\left(g\left(a_{(k) 4}^{l}\right)+g\left(a_{(k) 4}^{\prime l}\right)\right)\right)\right) ; \\
& g^{-1}\left(\sum_{k=1}^{n}\left(\mu\left(Z_{(k)}\right)-\mu\left(Z_{(k+1)}\right)\right) g\left(g^{-1}\left(g\left(h_{1}\left(A_{(k)}^{l}\right)\right)+g\left(h_{1}\left(A_{(k)}^{\prime l}\right)\right)\right)\right)\right), \\
& \left.\left.g^{-1}\left(\sum_{k=1}^{n}\left(\mu\left(Z_{(k)}\right)-\mu\left(Z_{(k+1)}\right)\right) g\left(g^{-1}\left(g\left(h_{2}\left(A_{(k)}^{l}\right)\right)+g\left(h_{2}\left(A_{(k)}^{\prime l}\right)\right)\right)\right)\right)\right)\right) \\
& =\left(\left(g^{-1}\left(\sum_{k=1}^{n}\left(\mu\left(Z_{(k)}\right)-\mu\left(Z_{(k+1)}\right)\right)\left(g\left(a_{(k) 1}^{u}\right)+g\left(a_{(k) 1}^{\prime u}\right)\right)\right),\right.\right. \\
& g^{-1}\left(\sum_{k=1}^{n}\left(\mu\left(Z_{(k)}\right)-\mu\left(Z_{(k+1)}\right)\right)\left(g\left(a_{(k) 2}^{u}\right)+g\left(a_{(k) 2}^{\prime u}\right)\right)\right), \\
& g^{-1}\left(\sum_{k=1}^{n}\left(\mu\left(Z_{(k)}\right)-\mu\left(Z_{(k+1)}\right)\right)\left(g\left(a_{(k) 3}^{u}\right)+g\left(a_{(k) 3}^{\prime \prime}\right)\right)\right), g^{-1}\left(\sum_{k=1}^{n}\left(\mu\left(Z_{(k)}\right)-\mu\left(Z_{(k+1)}\right)\right)\left(g\left(a_{(k) 4}^{u}\right)+g\left(a_{(k) 4}^{\prime \prime}\right)\right)\right) ; \\
& g^{-1}\left(\sum_{k=1}^{n}\left(\mu\left(Z_{(k)}\right)-\mu\left(Z_{(k+1)}\right)\right)\left(g\left(h_{1}\left(A_{(k)}^{u}\right)\right)+g\left(h_{1}\left(A_{(k)}^{\prime u}\right)\right)\right)\right), \\
& \left.g^{-1}\left(\sum_{k=1}^{n}\left(\mu\left(Z_{(k)}\right)-\mu\left(Z_{(k+1)}\right)\right)\left(g\left(h_{2}\left(A_{(k)}^{u}\right)\right)+g\left(h_{2}\left(A_{(k)}^{\prime u}\right)\right)\right)\right)\right) \text {, } \\
& \left(g^{-1}\left(\sum_{k=1}^{n}\left(\mu\left(Z_{(k)}\right)-\mu\left(Z_{(k+1)}\right)\right)\left(g\left(a_{(k) 1}^{l}\right)+g\left(a_{(k) 1}^{\prime l}\right)\right)\right), g^{-1}\left(\sum_{k=1}^{n}\left(\mu\left(Z_{(k)}\right)-\mu\left(Z_{(k+1)}\right)\right)\left(g\left(a_{(k) 2}^{l}\right)+g\left(a_{(k) 2}^{\prime l}\right)\right)\right),\right. \\
& g^{-1}\left(\sum_{k=1}^{n}\left(\mu\left(Z_{(k)}\right)-\mu\left(Z_{(k+1)}\right)\right)\left(g\left(a_{(k) 3}^{l}\right)+g\left(a_{(k) 3}^{\prime l}\right)\right)\right), g^{-1}\left(\sum_{k=1}^{n}\left(\mu\left(Z_{(k)}\right)-\mu\left(Z_{(k+1)}\right)\right)\left(g\left(a_{(k) 4}^{l}\right)+g\left(a_{(k) 4}^{\prime l}\right)\right)\right) ; \\
& g^{-1}\left(\sum_{k=1}^{n}\left(\mu\left(Z_{(k)}\right)-\mu\left(Z_{(k+1)}\right)\right)\left(g\left(h_{1}\left(A_{(k)}^{l}\right)\right)+g\left(h_{1}\left(A_{(k)}^{\prime l}\right)\right)\right)\right), g^{-1}\left(\sum_{k=1}^{n}\left(\mu\left(Z_{(k)}\right)-\mu\left(Z_{(k+1)}\right)\right)\right. \\
& \left.\left.\left.\left(g\left(h_{2}\left(A_{(k)}^{l}\right)\right)+g\left(h_{2}\left(A_{(k)}^{\prime l}\right)\right)\right)\right)\right)\right)=\operatorname{IT} 2 F A C\left(A_{1}, A_{2}, \ldots, A_{n}\right) \otimes I T 2 F A C\left(A_{1}^{\prime}, A_{2}^{\prime}, \ldots, A_{n}^{\prime}\right) .
\end{aligned}
$$




\section{Author Contributions}

The first author (Guolin Tang): Conceptualization, Formal analysis, Methodology, Validation, Writing original draft.

The second author (Francisco Chiclana): Formal analysis, Methodology, Construct, Resources, Writing - review \& editing

The third author (Xiangchun Lin): Data curation, Investigation, Resources, Visualization, Writing review \& editing

The third author (Peide Liu): Conceptualization, Formal analysis, Methodology, Funding acquisition, Supervision, Writing - review \& editing 